NBER WORKING PAPER SERIES

\title{
UNCERTAINTY AND ECONOMIC ACTIVITY: A MULTI-COUNTRY PERSPECTIVE
}

\author{
Ambrogio Cesa-Bianchi \\ M. Hashem Pesaran \\ Alessandro Rebucci \\ Working Paper 24325 \\ http://www.nber.org/papers/w24325 \\ NATIONAL BUREAU OF ECONOMIC RESEARCH \\ 1050 Massachusetts Avenue \\ Cambridge, MA 02138 \\ February 2018
}

We would like to thank Alex Chudik, Frank Diebold, Vadim Elenev, Domenico Giannone, Nicola Fusari, Michele Lenza, Pierre Noual, Giorgio Primiceri, Barbara Rossi, Ron Smith, Zhaogang Song, Allan Timmermann, and Paolo Zaffaroni for comments and useful suggestions. We have also benefited from comments by participants at the NBER Summer Institute (Forecasting and Empirical Methods Group), the 2017 BGSE Summer Forum, the ASSA Meetings, the EABCN-PWC-EUI Conference on "Time-varying models for monetary policy and financial stability," the 2017 International Conference on Computational and Financial Econometrics, the University of St Andrews "Workshop on Time-Varying Uncertainty in Macro," and seminars at the Bank of England and Johns Hopkins University. The views expressed in this paper are solely those of the authors and should not be taken to represent those of the Bank of England or the National Bureau of Economic Research.

NBER working papers are circulated for discussion and comment purposes. They have not been peer-reviewed or been subject to the review by the NBER Board of Directors that accompanies official NBER publications.

(C) 2018 by Ambrogio Cesa-Bianchi, M. Hashem Pesaran, and Alessandro Rebucci. All rights reserved. Short sections of text, not to exceed two paragraphs, may be quoted without explicit permission provided that full credit, including $\odot$ notice, is given to the source. 
Uncertainty and Economic Activity: A Multi-Country Perspective

Ambrogio Cesa-Bianchi, M. Hashem Pesaran, and Alessandro Rebucci

NBER Working Paper No. 24325

February 2018

JEL No. E44,F44,G12,G15

\begin{abstract}
$\underline{\text { ABSTRACT }}$
Measures of economic uncertainty are countercyclical, but economic theory does not provide definite guidance on the direction of causation between uncertainty and the business cycle. This paper proposes a new multi-country approach to the analysis of the interaction between uncertainty and economic activity, without a priori restricting the direction of causality. We develop a multi-country version of the Lucas tree model with time-varying volatility and show that in addition to common technology shocks that affect output growth, higher-order moments of technology shocks are also required to explain the cross country variations of the realized volatility of equity returns. Using this theoretical insight, two common factors, a 'real' and a 'financial' one, are identified in the empirical analysis assuming different patterns of crosscountry correlations of country-specific innovations to real GDP growth and realized stock market volatility. We then quantify the absolute and the relative importance of the common factor shocks as well as country-specific volatility and GDP growth shocks. The paper highlights three main empirical findings. First, it is shown that most of the unconditional correlation between volatility and growth can be accounted for by the real common factor, which is proportional to world growth in our empirical model and linked to the risk-free rate. Second, the share of volatility forecast error variance explained by the real common factor and by country-specific growth shocks amounts to less than 5 percent. Third, shocks to the common financial factor explain about 10 percent of the growth forecast error variance, but when such shocks occur, their negative impact on growth is large and persistent. In contrast, country-specific volatility shocks account for less than 1-2 percent of the growth forecast error variance.
\end{abstract}

Ambrogio Cesa-Bianchi

Bank of England

Threadneedle Street

London

EC2R 8AH

ambrogio.cesa-bianchi@bankofengland.co.uk

\author{
Alessandro Rebucci \\ Johns Hopkins Carey Business School \\ 100 International Drive \\ Baltimore, MD 21202 \\ and NBER \\ arebucci@jhu.edu
}

M. Hashem Pesaran

University of Southern California

pesaran@usc.edu 


\section{Introduction}

It is well-established that empirical measures of uncertainty behave countercyclically in the US and most other countries. ${ }^{1}$ This negative correlation can be seen in Figure 1 which shows the country-specific contemporaneous correlations between stock market realized volatility and real GDP growth for all countries in our panel together with their 95-percent error band. As can be seen, for most countries, there is a strong negative association between volatility and GDP growth. On average, this correlation is about -0.3 , ranging from a maximum of slightly more than -0.5 for Argentina to a minimum of just above zero for Peru. These correlations are statistically significant in the case of most countries, with the exception of Austria, China, Indonesia, Peru, and South Africa.

Figure 1 Country-Specific Correlations Between Volatility and Growth

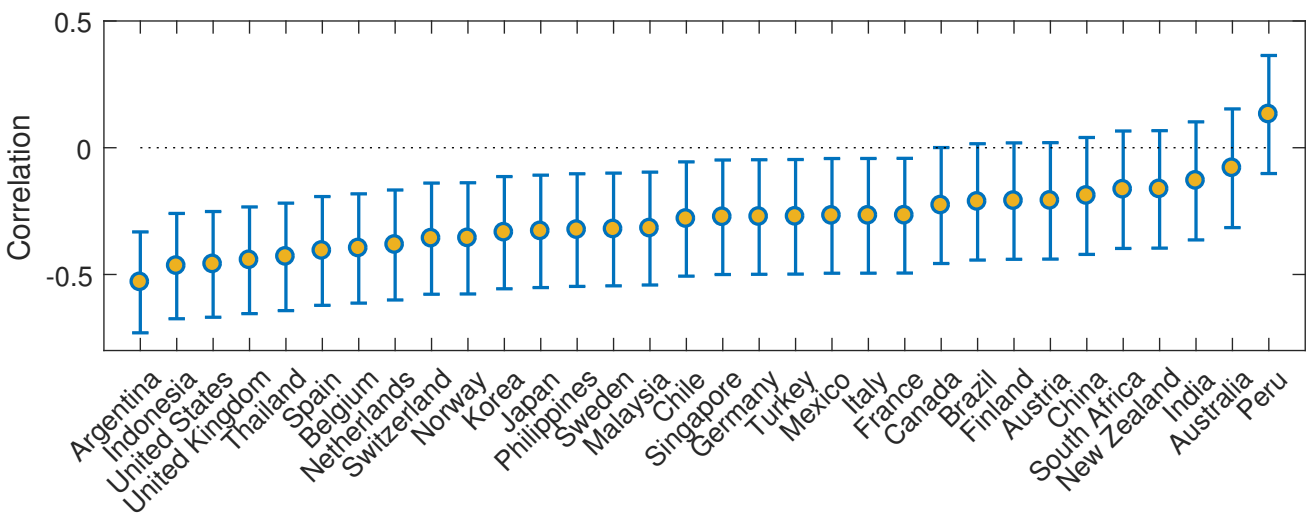

Note. Correlations between (log) realized stock market volatility and real GDP growth. The dots represent the country-specific contemporaneous correlations, and the lines represent $95 \%$ confidence intervals. See equation (64) in Section 5 for a definition of realized volatility at quarterly frequency and Section 6 for a description of the data. Sample period: 1993:Q1-2011:Q2.

Interpreting correlations in economic terms is difficult because causation can run in both directions. From a theoretical standpoint, uncertainty can cause economic activity to slowdown and even contract through a variety of mechanisms, both on the household side via precautionary savings (Kimball, 1990) and on the firm side via investment delays or other forms of frictions

\footnotetext{
${ }^{1}$ For the evidence on the US see, for example, Schwert (1989a) and Schwert (1989b) using the volatility of aggregate stock market returns; Campbell et al. (2001), Bloom et al. (2007), and Gilchrist et al. (2013) using the volatility of firm-level stock returns; Bloom et al. (2012) and Bachmann and Bayer (2013) using the volatility of plant, firm, industry and aggregate output and productivity; Popescu and Smets (2010) and Bachmann et al. (2013) using the behavior of expectations' disagreement. For the evidence on other countries see, for instance, Baker and Bloom (2013), Carriere-Swallow and Cespedes (2013), and Nakamura et al. (2017).
} 
(see for instance Bernanke (1983), Dixit and Pindyck (1994) and, more recently, Bloom (2009)), or financial frictions (Christiano et al., 2014, Gilchrist et al., 2013, Arellano et al., 2012). ${ }^{2}$ But it is also possible that uncertainty responds to fluctuations in economic activity. For instance, Bansal et al. (2005) show that fluctuations in expected growth directly affect asset valuations, and information regarding future expected growth is encoded in current asset valuations. Indeed, the theoretical literature highlights mechanisms through which spikes in uncertainty may be the result of adverse economic conditions. Examples based on information and financial frictions include Van Nieuwerburgh and Veldkamp (2006), Fostel and Geanakoplos (2012), and Ilut et al. (2017). ${ }^{3}$ Theory, therefore, does not provide a definite guidance as how to interpret the countercyclical nature of empirical measures of uncertainty. In this paper, we employ a multi-country approach to the analysis of interaction between uncertainty and economic activity, without restricting the direction of economic causation a priori.

We first develop a simple multi-country version of the Lucas (1978) tree model with timevarying volatility in which output or dividend growth rates are determined by a global technology (or global growth) factor. We show that country-specific equity returns are driven by two shocks, the first being the innovation to the global technology factor and the second being the innovation to its volatility. In effect, we develop a consumption-based international asset pricing model where at least two factors are needed to explain the cross country differences of equity returns, even though only one factor is sufficient to explain cross country differences in output growth.

This theoretical insight forms the basis of our econometric identification strategy. We measure uncertainty and activity with realized equity market volatility and real GDP growth, and assume that volatility and growth can be driven by two common factors, as well as countryspecific volatility and growth shocks. We identify the two common factors by assuming that innovations to volatility and growth have different patterns of correlations across countries. Specifically, we suppose that one of the factors, which we label as "real", is sufficient to model cross-country correlations of output growth innovations, but a second factor, which we label as "financial", is also needed to model the cross-country correlations of volatility innovations.

\footnotetext{
${ }^{2}$ Pricing frictions and the zero lower bound on nominal interest rates can also amplify the impact of a volatility shock (Fernandez-Villaverde et al. (2011), Basu and Bundick (2017), Born and Pfeifer (2014)).

${ }^{3}$ Theoretically, the impact of uncertainty on activity could even be positive. For example Mirman (1971) shows that, if there is a precautionary motive for savings, then higher volatility would lead to higher investments. Oi (1961), Hartman (1976) and Abel (1983) show that if labor can be freely adjusted, the marginal revenue product of capital is convex in price; in this case, uncertainty may increase the level of the capital stock and, therefore, investment. However, these theories are not consistent with the countercyclical nature of uncertainty measures.
} 
Specifically, we assume weak cross-country dependence of growth innovations and strong crosscountry dependence of volatility innovations (in the sense of Chudik et al., 2011). This is equivalent to assuming that volatility innovations share at least one more strong common factor than growth innovations. As the paper shows, under this condition, the real common factor is proportional to world GDP growth, measured as the weighted average of country-specific growth rates. ${ }^{4}$ Also, if it is further assumed that the volatility series share only one additional strong factor as compared to the growth innovations, the loading matrix on the global factors becomes triangular, with country-specific volatilities loading contemporaneously on both the real and financial factor, and country growth rates loading only on the real factor. Under this condition, the second factor, common only to the volatility series, can then be identified in our model and measured in the data as the residual of a OLS regression of world volatility on world GDP growth.

Our identification assumptions are in accordance with patterns of cross-country correlation that we document in the data, as well as statistical tests of the cross-country dependence of the estimated country-specific volatility and growth innovations. For instance, for each country in our sample, Figure 2 plots the average pair-wise correlation of volatility and output growth series, together with the average across all countries. ${ }^{5}$ It can be seen that the average pair-wise correlation across all countries for the volatility series is more than twice the average for the growth series, at 0.58 and 0.27 , respectively (the two dotted lines). This evidence suggests that, indeed, the volatility series are much more correlated across countries than the growth series. ${ }^{6}$ We also find even more striking differences when we consider the cross-country dependence of the volatility and growth innovations (See Section 7.2).

To measure economic uncertainty, we build on the contributions of Andersen et al. (2001, 2003) and Barndorff-Nielsen and Shephard (2002, 2004) and compute realized equity price volatility for a given quarter by using daily returns for 32 advanced and emerging economies representing more than 90 percent of the world economy. We also consider several other proxies for uncertainty and argue that they are either not suitable for the purpose of our analysis, or

\footnotetext{
${ }^{4}$ Our identification assumption is compatible with the view that the global financial cycle (e.g. Rey, 2013) is stronger than the international business cycle (e.g., Kose et al. (2003)), with realized equity market volatility co-moving more closely across countries than real GDP growth does.

${ }^{5}$ The average pair-wise correlation of a variable $x$ for country $i$ (i.e., each bar in Figure 2) is defined as the average bilateral correlation of $x_{i t}$ with $x_{j t}$ for all $j \neq i$. See equation (65) in Section 6 for a more formal definition.

${ }^{6}$ We note here that these patterns of cross-country correlations are consistent with those documented by Tesar (1995), Colacito and Croce (2011), and Lewis and Liu (2015) for consumption growth and equity returns, respectively.
} 
Figure 2 Average Pair-Wise Correlations of Volatility and Growth

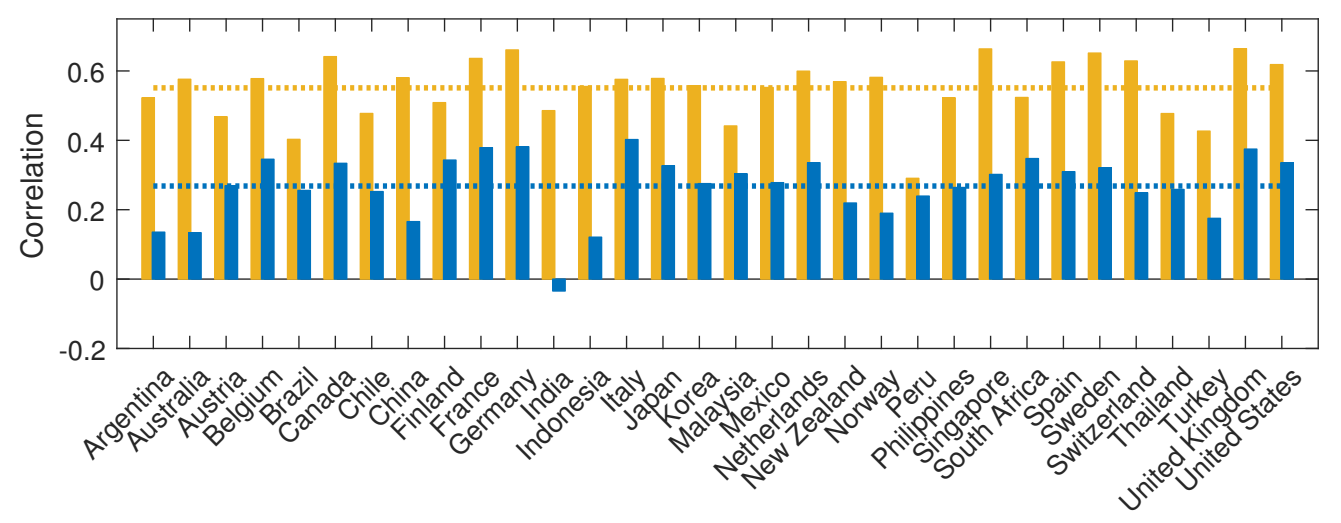

Note. For each country, the light (yellow) and the dark (blue) bar represent the average pair-wise correlation with the remaining countries in the sample for volatility and GDP growth series, respectively. The dotted lines correspond to the overall average across all countries, equal to 0.55 and 0.27 for volatility and GDP growth, respectively. The average pair-wise correlation of a variable $x_{i t}$ in country $i$ is the average of the contemporaneous correlation between $x_{i t}$ and $x_{j t}$ for all $j \neq i$. See equation (64) in Section 5 for a definition of the realized volatility measure and Section 6 for a description of the data. Sample period: 1993:Q1-2011:Q2.

not readily available for a large number of countries over a sufficiently long period needed for our analysis, or that they are closely associated with realized volatility.

The empirical analysis yields a rich set of findings. Here we highlight three main results. First, and most importantly, we find that the bulk of the negative correlations between volatility and output growth observed in the data can be accounted for by the real common factor. While unconditionally volatility behaves countercyclically for all but one of the 32 countries in our sample, when we condition on the real factor, the correlations between volatility and growth innovations become statistically insignificant in all but two emerging economies, and quantitatively much smaller in all countries (changing sign in more than half of the cases). This result does not depend on any auxiliary assumptions made, including that volatility series share only one additional strong factor, and suggests that part of the explanatory power attributed to uncertainty shocks in empirical studies of individual countries, considered in isolation from the rest of the world economy, might be due to omitting such a real common factor from the analysis.

Second, the paper shows that the time-variation of country-specific volatility is explained largely by shocks to the financial factor (with a share of forecast error variance larger than 60\%) and innovations to country-specific volatility series themselves (with a share of forecast error variance of about 35 percent). Shocks to the real common factor and to country-specific growth 
innovations explain less than 5 percent of volatility forecast error variance. We interpret this evidence as suggesting that the endogenous component of country-specific volatility is small, or, equivalently, that volatility measured at quarterly frequency is largely an exogenous process.

Third and finally, we find that shocks to the common financial factor explain about $10 \%$ of the forecast error variance of output growth, even though they have strong and persistent contractionary effects. In contrast, country-specific volatility shocks explain only $1-2$ percent of country-specific forecast error growth variance. These results illustrate the quantitative importance of distinguishing between common and country-specific volatility shocks. In our empirical model, the forecast error variance of output growth is explained mainly by innovations to country-specific growth rates themselves (with a share of at least $60 \%$ percent) and the real common factor (with another $25 \%$ percent of the total).

The paper is closely related to three strands of empirical literature on volatility and growth. ${ }^{7}$ The first strand acknowledges that uncertainty may be endogenous and could be driven by the business cycle (See, for instance, Ludvigson et al. (2015), Clark et al. (2016), and Berger et al. (2017)). ${ }^{8}$ A key difference relative to these contributions, is that our identification assumptions apply to a cross-section of countries, as opposed to a single country considered in isolation from the rest of the world, or the global economy analyzed as a single, closed economy. Also, our identification strategy is simpler and consistent with observable cross-country correlation properties of the data and the estimated country-specific innovations as opposed to unobservable theoretical conditions. Interestingly, despite the different approaches taken to proxy for uncertainty and to separate endogenous responses to the business cycle from exogenous changes, we reach similar conclusions that the endogenous component of country-specific volatility is small, and exogenous shifts in uncertainty can be quite harmful for output growth. According to our analysis, however, the latter applies only to the global component of uncertainty that cannot be identified separately in the empirical frameworks that focus on individual countries, taken in isolation from the rest of the world economy.

A second strand of the literature has an international focus as in our paper. For instance, Carriere-Swallow and Cespedes (2013) estimate a battery of 40 small open economy VARs for advanced and emerging economies in which the US VIX index is assumed to be exogenous and

\footnotetext{
${ }^{7}$ The literature is voluminous. See Bloom (2014) for a recent survey. Here we focus only on studies directly related to our paper.

${ }^{8}$ Diebold and Yilmaz (2010) is an early attempt to separate macroeconomic and financial uncertainty without addressing endogeneity issues.
} 
identification is achieved imposing country-by-country restrictions. Baker and Bloom (2013) study an unbalanced panel of 60 countries, documenting the counter-cyclicality of different proxies for uncertainty, such as stock market volatility, sovereign bond yields volatility, exchange rate volatility and GDP forecast disagreement, and use measures of disaster risk as instruments. Hirata et al. (2012) estimate a factor-augmented VAR (FAVAR), with factors computed based on data for 18 advanced economies, and use a recursive identification scheme in which the volatility variable is ordered first in the VAR. Carriero et al. (2017) estimate a large Bayesian VAR with exogenously driven stochastic volatility to quantify the impact of macroeconomic uncertainty on OECD economies. Hirata et al. (2012), Carriere-Swallow and Cespedes (2013), Carriero et al. (2017) therefore, restrict the direction of economic causation from the outset of the analysis assuming that the uncertainty proxy used is exogenous. In addition, in our framework, countries interact with each other not only via the common factors, but also via an unrestricted variance-covariance matrix of the country-specific volatility and growth innovations. In contrast, in the above studies, economies can interact only via common factors or variables like the VIX index, but do not interact with each other via other spillover channels.

Our paper also relates to contributions in the finance literature. The closest analogous to the framework we propose are mean-variance frontier models-discussed, for example, by Black (1976) and French et al. (1987). In those models, however, the focus is on possible causal relations between the stock market return and its volatility, via leverage effect or other channels. We model the contemporaneous relation between country-specific GDP growth and stock market volatility. We argue that one can think of GDP as the 'dividend' or the 'cash flow' associated with the country stock market index. In this sense, the novelty of our modeling approach is to work in the dividend-volatility (or cash flow-volatility) space rather than return-volatility space. Indeed, our identification strategy exploits the fact that country-specific dividend growth processes (the country GDP growth rates) are less correlated across countries as compared to the cross-market correlation of equity volatilities.

The rest of the paper is organized as follows. Section 2 sets out the theoretical multi-country model and derives country-specific equity returns and volatilities and shows how they are related to the underlying world technology factor and its higher moments. Section 3 considers the econometric issues involved in identification of the real and financial factors using a static version of our econometric multi-country model. Section 4 extends the analysis to a dynamic setting. Section 5 considers the use of realized volatility as a proxy measure for uncertainty in 
a multi-country setting. Section 6 reports key stylized facts of the data, including evidence on the cross-country correlation structure of volatilities and output growth. Section 7 reports the main empirical results of the paper on the comparison between unconditional and conditional correlations between volatility and growth. Section 8 reports forecast error variance decompositions and discusses the corresponding empirical results. Section 9 presents impulse responses, and Section 10 concludes. Some of the technical proofs and details of the data and their sources are provided in the Appendix. Derivation of impulse responses and variance decompositions, together with additional empirical results as well as selected country-specific results are reported in a separate online supplement to the paper.

Notations: Let $\mathbf{w}=\left(w_{1}, w_{2}, \ldots, w_{n}\right)^{\prime}$ and $\mathbf{A}=\left(a_{i j}\right)$ be an $n \times 1$ vector and an $n \times n$ matrix,

respectively, and denote the largest eigenvalue of $\mathbf{A}$, by $\varrho_{\max }(\mathbf{A})$. Then, $\|\mathbf{w}\|=\left(\Sigma_{i=1}^{n} w_{i}^{2}\right)^{1 / 2}$ and $\|\mathbf{A}\|=\left[\varrho_{\max }\left(\mathbf{A}^{\prime} \mathbf{A}\right)\right]^{1 / 2}$ are the Euclidean $\left(L_{2}\right)$ norm of $\mathbf{w}$, and the spectral norm of $\mathbf{A}$, respectively. $\boldsymbol{\tau}_{T}$ is a $T \times 1$ vector of ones, $\boldsymbol{\tau}_{T}=(1,1, \ldots, 1)^{\prime}$. If $\left\{y_{n}\right\}_{n=1}^{\infty}$ is any real sequence and $\left\{x_{n}\right\}_{n=1}^{\infty}$ is a sequences of positive real numbers, then $y_{n}=O\left(x_{n}\right)$, if there exists a positive finite constant $C_{0}$ such that $\left|y_{n}\right| / x_{n} \leq C_{0}$ for all $n$. $y_{n}=o\left(x_{n}\right)$ if $f_{n} / g_{n} \rightarrow 0$ as $n \rightarrow \infty$. If $\left\{y_{n}\right\}_{n=1}^{\infty}$ and $\left\{x_{n}\right\}_{n=1}^{\infty}$ are both positive sequences of real numbers, then $y_{n}=\mathcal{O}\left(x_{n}\right)$ if there exists $N_{0} \geq 1$ and positive finite constants $C_{0}$ and $C_{1}$, such that $\inf _{n \geq N_{0}}\left(y_{n} / x_{n}\right) \geq C_{0}$, and $\sup _{n \geq N_{0}}\left(y_{n} / x_{n}\right) \leq C_{1}$. By "granular" we mean "asymptotically small" in the sense of Chudik and Pesaran (2013).

\section{Equity Returns and Volatility in a Multi-country Business Cycle Model}

In this section we set up a simple theoretical model of the business cycle with time-varying volatility that will help to guide the empirical analysis. To this end we consider a multi-country version of the Lucas (1978) tree model augmented with time-varying volatility that establishes a link between changes in volatility and business cycle fluctuations via a common (global) risk-free rate. Specifically, consider a world consisting of $N$ economies (countries) indexed by $i=1,2, \ldots N$, of similar but not necessarily identical relative sizes, $w_{i t}=O\left(N^{-1}\right)$, where $\Sigma_{i=1}^{N} w_{i t}=1$. We shall also assume that these economies have the same preferences, but are exposed differently to a world growth factor, assumed to be exogenously given. The world growth factor is largely, 
but not exclusively, driven by technological factors.

Each economy $i$ is inhabited by an infinitely-lived representative agent endowed with a stochastic stream of a single homogeneous good $Y_{i, t+s}, s=0,1,2, \ldots$, viewed as the economy's measure of real output or GDP. It is assumed that the country's output growth rate, $\Delta y_{i t}=$ $\ln \left(Y_{i t} / Y_{i, t-1}\right)$ fluctuates around a deterministic steady state, $g_{i}$, driven by country-specific, $\varepsilon_{i t}$, and global, $f_{t}$, shocks, namely

$$
\Delta y_{i t}=g_{i}+\gamma_{i} f_{t}+\varepsilon_{i t}
$$

Despite its simplicity, the assumed growth process is consistent with multi-country versions of the international real business cycle models of Backus et al. (1992) and Baxter and Crucini (1995), and it is at the core of typical new open-economy DSGE models. ${ }^{9}$

To simplify the exposition we assume that $\varepsilon_{i t}$ for $i=1,2, \ldots, N$ are serially uncorrelated and independently distributed across $i$. Also, without loss of generality, we assume that $f_{t}$ and $\varepsilon_{i t}$ are uncorrelated. However, richer time series dynamics, as well as weak forms of cross-country dependence, could be allowed for. Indeed, empirically, we model the dynamics of country-specific equity market volatility and the business cycle jointly as factor augmented vector autoregressive processes, weakly correlated across countries. But to simplify the derivations and obtain a closed form solution we assume $\varepsilon_{i t} \sim \operatorname{IIDN}\left(0, \sigma_{\varepsilon i}^{2}\right)$, and $f_{t}$ follows a stationary first order autoregressive process with conditionally heteroskedastic errors. More specifically

$$
f_{t}=\phi_{f} f_{t-1}+\nu_{t}
$$

where $\left|\phi_{f}\right|<1, \nu_{t} \sim \operatorname{IIDN}\left(0, \sigma_{\nu}^{2}\right)$, and

$$
\operatorname{Var}_{t-1}\left(\nu_{t}\right)=E_{t-1}\left(\nu_{t}^{2}\right)=a_{f}+b_{f} \nu_{t-1}^{2}
$$

with $a_{f}>0,0<b_{f}<1 . E_{t-1}()=.E\left(. \mid \mathfrak{I}_{t-1}\right)$ and $\operatorname{Var}_{t-1}()=.\operatorname{Var}\left(. \mid \mathfrak{I}_{t-1}\right)$ denoting conditional expectations and variance operators with respect to the non-decreasing information set, $\mathfrak{I}_{t-1}$, which contains at least all country specific variables as well as the global risk-free rate (see below for further details). Note here that $\nu_{t}$ is conditionally heteroskedastic, but unconditionally

\footnotetext{
${ }^{9}$ For instance, in section A.1 of the paper Appendix, we show that (1) can be justified as the ergodic limit process to which a stochastic multi-country version of the neoclassical growth model converges. In that case, $f_{t}$ in (1) can be interpreted as the (stochastic) rate of world technology growth. But in our empirical model it captures also other common forces driving world growth over the business cycle.
} 
homoskedastic with $\operatorname{Var}\left(\nu_{t}\right)=\sigma_{\nu}^{2}=a_{f} /\left(1-b_{f}\right)>0 .{ }^{10}$

The representative agent of country $i$ can trade freely a globally available risk-free bond and $N$ risky equity claims defined on the country-specific entire endowment streams, $Y_{i, t+s}$, for $s=0,1,2, \ldots \infty$. International asset markets are complete in Arrow-Debreau sense so that country-specific consumption growth is equalized across countries, and one can use the world endowment growth in the stochastic discount factor of country $i$ 's representative agent. ${ }^{11}$

The representative agent in country $i$ has constant relative risk aversion (CRRA) period utility function and maximizes lifetime utility,

$$
E_{t}\left[\sum_{s=t}^{\infty} \beta^{s}\left(\frac{C_{i s}^{1-\varrho}}{1-\varrho}\right)\right]
$$

where $\varrho>0$ is the coefficient of relative risk aversion (with $\varrho \neq 1$ ) and $\beta$ is the subjective discount factor, both common across countries. The period budget constraint is:

$$
C_{i t}+B_{i, t+1}+\sum_{j=1}^{N} \theta_{i, t+1}^{(j)} P_{j t}=\left(1+r_{t}^{f}\right) B_{i t}+\sum_{j=1}^{N} \theta_{i t}^{(j)}\left(Y_{j t}+P_{j t}\right)
$$

where $C_{i t}$ is consumption of country $i$ during period $t, B_{i t}$ is the risk-free bond held by country $i$ at the start of period $t$, with real gross return $1+r_{t}^{f} \cdot \theta_{i t}^{(j)}$ is the share of country $j^{\text {th }}$ income stream held by the representative agent of country $i$ at the start of period $t$, with ex-dividend market value $P_{j t}$, subject to the adding-up constraints $\sum_{i=1}^{N} \theta_{i t}^{(j)}=1$, for $j=1,2, \ldots N .^{12}$ Substituting for $C_{i t}$ from (5) in (4), the first order conditions for choosing the bond holding $B_{i, t+1}$, and the $N$ equity holdings, $\theta_{i, t+1}^{(j)}$, are:

$$
1+r_{t+1}^{f}=\frac{1}{E_{t}\left[\beta\left(\frac{C_{i, t+1}}{C_{i t}}\right)^{-\varrho}\right]}, \text { for } i=1,2, \ldots, N
$$

\footnotetext{
${ }^{10}$ For further clarity, we will refer to $f_{t}$ as the "growth factor", and to $\nu_{t}$ as the "innovation" or "shock" to the growth factor.

${ }^{11}$ In this set up, one could prove that asset market are complete in Arrow-Debreau rather than assuming it if we were to restrict the specification of the stochastic processes for $\varepsilon_{i t}$ and $f_{t}$ such that the number of uncertain states of the world is less than $N+1$. See for instance Chapter 5 of Obstfeld and Rogoff (1996) and Aiyagari (1993)).

${ }^{12}$ Note that the risk-free rate, $r_{t+1}^{f}$, is known at the start of period $t$, and hence included in the information set $\mathfrak{I}_{t}$.
} 
and

$$
P_{j t}=E_{t}\left\{\left[\beta\left(\frac{C_{i, t+1}}{C_{i t}}\right)^{-\varrho}\right]\left(P_{j, t+1}+Y_{j, t+1}\right)\right\}, \text { for } i, j=1,2, \ldots, N .
$$

Note that since by assumption the equity markets are complete in the Arrow-Debreu sense, in the first order conditions above, the stochastic discount factor for country $i$ can be set as

$$
E_{t}\left[\left(\frac{C_{i, t+1}}{C_{i t}}\right)^{-\varrho}\right]=E_{t}\left[\left(\frac{Y_{w, t+1}}{Y_{w t}}\right)^{-\varrho}\right]=E_{t}\left[\exp \left(-\varrho \Delta \ln Y_{w, t+1}\right)\right]
$$

where $Y_{w, t+1}$ is the world output, defined by $Y_{w, t+1}=\sum_{i=1}^{N} Y_{i, t+1} \cdot{ }^{13}$ Therefore, the above first-order conditions, (6) and (7), can be written as

$$
E_{t}\left[\beta \exp \left(-\varrho \Delta \ln Y_{w, t+1}\right)\right]=\frac{1}{1+r_{t+1}^{f}},
$$

and

$$
E_{t}\left\{R_{i, t+1}\left[\beta\left(\frac{C_{i, t+1}}{C_{i t}}\right)^{-\varrho}\right]\right\}=1, \text { for } i=1,2, \ldots, N,
$$

where $R_{i, t+1}$ is the gross return on country $i^{t h}$ endowment defined by $R_{i, t+1}=\left(P_{i, t+1}+Y_{i, t+1}\right) / P_{i t}$.

\subsection{Derivation of the Risk-free Rate}

We now use the above first order conditions to relate the growth factor, $f_{t}$, to the asset returns. We begin with the risk-free rate and using (1), we note that

$$
\Delta \ln Y_{w, t+1}=\ln \left(1+g_{w, t+1}\right)
$$

where $g_{w, t+1}=\left(\sum_{i=1}^{N} Y_{i, t+1} / \sum_{i=1}^{N} Y_{i t}\right)-1$ is the world output growth, which can also be written equivalently as

$$
g_{w, t+1}=\frac{\sum_{i=1}^{N}\left(Y_{i, t+1}-Y_{i t}\right)}{\sum_{i=1}^{N} Y_{i t}}=\frac{\sum_{i=1}^{N} Y_{i t} g_{i, t+1}}{\sum_{i=1}^{N} Y_{i t}}=\sum_{i=1}^{N} w_{i t} g_{i, t+1},
$$

where $g_{i, t+1}=\left(Y_{i, t+1} / Y_{i t}\right)-1$, for $i=1,2, \ldots, N$ are country-specific growth rates, and $w_{i t}=$ $Y_{i t} / \sum_{j=1}^{N} Y_{j t}$ is the size of country $i$ in the world economy at time $t$. Also since $g_{i, t+1}$ and $g_{w, t+1}$

\footnotetext{
${ }^{13}$ See for instance Chapter 5 of Obstfeld and Rogoff (1996) and Aiyagari (1993).
} 
are small they can be well approximated by

$$
\begin{aligned}
g_{w, t+1} & \approx \ln \left(1+g_{w, t+1}\right)=\Delta \ln \left(Y_{w, t+1}\right) \\
g_{i, t+1} & \approx \ln \left(1+g_{i, t+1}\right)=\Delta \ln \left(Y_{i, t+1}\right)=\Delta y_{i, t+1}
\end{aligned}
$$

which yields

$$
g_{w, t+1} \approx \Delta \ln \left(Y_{w, t+1}\right) \approx \sum_{i=1}^{N} w_{i t} \Delta y_{i, t+1} .
$$

Using this result in (8) and then in (9) now yields

$$
1+r_{t+1}^{f} \approx \frac{1}{E_{t}\left[\beta \exp \left(-\varrho \sum_{i=1}^{N} w_{i t} \Delta y_{i, t+1}\right)\right]}
$$

Finally, using the country-specific output growth equations (1) we also have

$$
\sum_{i=1}^{N} w_{i t} \Delta y_{i, t+1}=\sum_{i=1}^{N} w_{i t}\left(g_{i}+\gamma_{i} f_{t+1}+\varepsilon_{i, t+1}\right)=g_{w t}+\gamma_{w t} f_{t+1}+\varepsilon_{w, t+1},
$$

where $g_{w t}=\sum_{i=1}^{N} w_{i t} g_{i}, \gamma_{w t}=\sum_{i=1}^{N} w_{i t} \gamma_{i}$, and $\varepsilon_{w, t+1}=\sum_{i=1}^{N} w_{i t} \varepsilon_{i, t+1}$. Note that $g_{w t}$ and $\gamma_{w t}$ are included in the information set $\mathfrak{I}_{t}$. Under the assumptions that $f_{t+1}$ and $\varepsilon_{i, t+1}$ for $i=1,2, \ldots, N$ are Gaussian, then conditional on $\mathfrak{I}_{t}, \Delta y_{w, t+1}$ is also Gaussian and we have:

$$
\begin{aligned}
E_{t}\left[\exp \left(-\varrho \sum_{i=1}^{N} w_{i t} \Delta y_{i, t+1}\right)\right] & =e^{-\varrho g_{w t}} E_{t}\left(e^{-\varrho \gamma_{w t} f_{t+1}-\varrho \varepsilon_{w, t+1}}\right) \\
& =e^{-\varrho g_{w t}-\varrho \gamma E_{t}\left(f_{t+1}\right)+\frac{1}{2}\left[\varrho^{2} \gamma_{w t}^{2} \operatorname{Var}_{t}\left(f_{t+1}\right)+\varrho^{2} \operatorname{Var}_{t}\left(\varepsilon_{w, t+1}\right)\right]} .
\end{aligned}
$$

Setting $\beta=1 /(1+r))$ and using the above result in (11) we obtain

$$
\ln \left(\frac{1+r_{t+1}^{f}}{1+r}\right)=\varrho g_{w t}+\varrho \gamma E_{t}\left(f_{t+1}\right)-\frac{\varrho^{2}}{2}\left[\gamma_{w t}^{2} \operatorname{Var}_{t}\left(f_{t+1}\right)+\operatorname{Var}_{t}\left(\varepsilon_{w, t+1}\right)\right]
$$

But under (2) and (3),

$$
E_{t}\left(f_{t+1}\right)=\phi_{f} f_{t}, \text { and } \operatorname{Var}_{t}\left(f_{t+1}\right)=a_{f}+b_{f} \nu_{t}^{2}
$$


Furthermore, since by assumption the idiosyncratic shocks, $\varepsilon_{i t}$, are cross-sectionally independent and $w_{i t}=O\left(N^{-1}\right)$, we also have $\operatorname{Var}_{t}\left(\varepsilon_{w, t+1}\right)=O\left(N^{-1}\right)$. Therefore, overall we have:

$$
r_{t+1}^{f} \approx\left(r+\varrho g_{w t}-\frac{1}{2} \varrho^{2} \gamma_{w t}^{2} a_{f}\right)+\left(\gamma \varrho \phi_{f}\right) f_{t}-\frac{1}{2}\left(\varrho^{2} \gamma_{w t}^{2} b_{f}\right) \nu_{t}^{2}+O\left(N^{-1}\right)
$$

This expression shows how the global risk-free rate responds to changes in the composition of world output growth, $g_{w t}$, the expected change in the level of the global growth factor, $\left(\gamma \varrho \phi_{f}\right) f_{t}$, and expected change in the volatility of the global factor, $\frac{1}{2}\left(\varrho^{2} \gamma_{w t}^{2} b_{f}\right) \nu_{t}^{2}$. An expected increase in level of growth factor increases the risk-free rate, whilst a rise in the expected volatility of the global factor reduces it.

We now show how the above equation for the risk-free rate can be used to relate equity return volatility and output growth, but to simplify the exposition we abstract from time variations in the weights and set $w_{i t}=w_{i}$. So in what follows we use the following simplified version of (14):

$$
r_{t+1}^{f} \approx\left(r+\varrho g-\frac{1}{2} \varrho^{2} \gamma^{2} a_{f}\right)+\left(\gamma \varrho \phi_{f}\right) f_{t}-\frac{1}{2}\left(\varrho^{2} \gamma^{2} b_{f}\right) \nu_{t}^{2}+O\left(N^{-1}\right)
$$

where $\gamma=\gamma_{w}=\sum_{i=1}^{N} w_{i} \gamma_{i}$, and $g=g_{w}=\sum_{i=1}^{N} w_{i} g_{i}$.

\subsection{Country Equity Returns and their Realized Volatility}

Consider now the first order conditions for the equity returns given by (10), which are non-linear in current and expected future output growth. To obtain an analytical solution we make use of the approximate present-value relation for stock market returns derived by Campbell and Shiller (1988) (CS, henceforth), and note that in our set up $D_{i t}=Y_{i t}$. Let $\kappa_{i t}=P_{i t} /\left(P_{i t}+Y_{i t}\right)$ and, following CS assume that $\kappa_{i t}$ is approximately constant over time and set to $\kappa_{i}$ with $0<\kappa_{i}<1$. Then using result (2') of CS we have

$$
r_{i, t+1}=\Delta y_{i, t+1}+\delta_{i t}-\kappa_{i} \delta_{i, t+1}
$$

where $r_{i, t+1}=\ln \left(R_{i, t+1}\right)=\ln \left(P_{i, t+1}+Y_{i, t+1}\right)-\ln \left(P_{i, t}\right)$ is the realized gross log-return on country $i^{t h}$ equity, $y_{i t}=\ln \left(Y_{i t}\right)$, and $\delta_{i t}=\ln \left(Y_{i, t} / P_{i t}\right) .{ }^{14}$ Further, CS show that irrespective of the asset pricing model considered, under rational expectations and assuming that the transversality

\footnotetext{
${ }^{14}$ In their derivations CS use $b_{t}, d_{t}$ and $r_{t}$, for our $r_{i, t+1}, d_{i, t+1}$ and $r_{t+1}^{f}$, respectively. See their equations (1) and (5) and the related discussion in CS.
} 
condition ruling out rational bubbles holds, using result (6) of CS, we also have

$$
\delta_{i t}=\sum_{j=0}^{\infty} \kappa_{i}^{j}\left[E_{t}\left(r_{t+j+1}^{f}\right)-E_{t}\left(\Delta y_{i, t+j+1}\right)\right]
$$

where $r_{t+1}^{f}$ is the (world) risk-free rate as given by (15). Using (1) and (15), we have

$$
E_{t}\left(\Delta y_{i, t+j+1}\right)=g_{i}+\gamma_{i} E_{t}\left(f_{t+j+1}\right)
$$

and

$$
E_{t}\left(r_{t+j+1}^{f}\right) \approx\left(r+\varrho g-\frac{1}{2} \varrho^{2} \gamma^{2} a_{f}\right)+\left(\gamma \varrho \phi_{f}\right) E_{t}\left(f_{t+j}\right)-\frac{1}{2}\left(\varrho^{2} \gamma^{2} b_{f}\right) E_{t}\left(\nu_{t+j}^{2}\right)+O\left(N^{-1}\right)
$$

Also using (2) and (3) it follows that

$$
E_{t}\left(f_{t+j}\right)=\phi_{f}^{j} f_{t}, \text { and } E_{t}\left(\nu_{t+j}^{2}\right)=\frac{\left(1-b_{f}^{j}\right) a_{f}}{1-b_{f}}+b_{f}^{j} \nu_{t}^{2}
$$

Substituting the above results in (17) and then in (16), after some algebra and lagging by one period we obtain ${ }^{15}$

$$
r_{i t}=a_{r}+\gamma \varrho \phi_{f} f_{t-1}-\frac{1}{2} \varrho^{2} \gamma^{2} b_{f} \nu_{t-1}^{2}+a_{i 0} \nu_{t}+b_{i 0} \chi_{t}+\varepsilon_{i t}+O\left(N^{-1}\right),
$$

where

$$
a_{r}=r+\varrho g, \nu_{t}=f_{t}-\phi_{f} f_{t-1} \text { and } \chi_{t}=\nu_{t}^{2}-a_{f}-b_{f} \nu_{t-1}^{2},
$$

and

$$
a_{i 0}=\frac{\gamma_{i}-\kappa_{i} \gamma \varrho \phi_{f}}{1-\kappa_{i} \phi_{f}}, \text { and } b_{i 0}=\frac{1}{2}\left(\frac{\kappa_{i} \varrho^{2} \gamma^{2} b_{f}}{1-\kappa_{i} b_{f}}\right)
$$

The above return equation has a number of interesting features. First, the returns are explicitly related to the innovations in the underlying growth factor, $f_{t}$, and its volatility. Second, the factor loadings in (21) vary across countries partly reflecting the different responsiveness of their growth process to $f_{t}$, as well as the relative importance of $D_{i t}$ in $P_{i t}+D_{i t}$, as captured by parameter $\kappa_{i}$. This heterogeneity is present even though the risk preference parameter, $\varrho$, is assumed to be identical across countries. Third, crucially for our empirical analysis, while

\footnotetext{
${ }^{15}$ Details of the derivations can be found in the Appendix, sub-section A.2. See equation (A.3).
} 
only one common shock is sufficient to explain cross country differences in output growth, at least two common shocks, $\nu_{t}$ and $\chi_{t}$, are required to explain the cross country differences of equity returns. ${ }^{16}$ The innovations $\nu_{t}$ and $\chi_{t}$, can be viewed as first and second order moment shocks, respectively. The conditional covariance of these two shocks is given by $\operatorname{Cov}_{t-1}\left(\nu_{t}, \chi_{t}\right)=$ $E_{t-1}\left(\nu_{t} \chi_{t}\right)=E_{t-1}\left(\nu_{t}^{3}\right)$, which measures the conditional asymmetry of the technology shock in our model. ${ }^{17}$

In our empirical application, we consider the relationship between output growth and realized volatility of equity returns across countries, computed from squares of daily returns within a quarter to match the available data on output growth-see Section 5 below. To link the above theoretical results to our empirical analysis, denote output growth and equity returns for a given day $\tau$ within a quarter $t$ with $\Delta y_{i t}(\tau)$, and $r_{i t}(\tau)$, respectively, for $\tau=1,2, \ldots, D$, where $D$ is the number of trading days within a quarter (which we assume to be fixed across $t$ for convenience). In this set up, the underlying daily growth factor and country-specific shocks are given by $f_{t}(\tau)$ and $\varepsilon_{i t}(\tau)$. So, in terms of daily changes, the theoretical output growth and equity return equations can be written as

$$
\Delta y_{i t}(\tau)=g_{i}(\tau)+\gamma_{i} f_{t}(\tau)+\varepsilon_{i t}(\tau)
$$

and

$$
r_{i t}(\tau)=a_{r}(\tau)+b_{r} f_{t-1}(\tau)+c_{r} \nu_{t-1}^{2}(\tau)+a_{i 0} \nu_{t}(\tau)+b_{i 0} \chi_{t}(\tau)+\varepsilon_{i t}(\tau)+O\left(N^{-1}\right)
$$

where $b_{r}=\gamma \varrho \phi_{f}$, and $c_{r}=-\frac{1}{2} \varrho^{2} \gamma^{2} b_{f}$. Using the above daily models of output growth and equity returns, the associated quarterly output growth rates and realized equity return volatilities (respectively) are

$$
\begin{aligned}
\Delta y_{i t} & =\sum_{\tau=1}^{D} g_{i}(\tau)+\gamma_{i} \sum_{\tau=1}^{D} f_{t}(\tau)+\sum_{\tau=1}^{D} \varepsilon_{i t}(\tau) \\
& =g_{i}+\gamma_{i} f_{t}+\varepsilon_{i t},
\end{aligned}
$$

\footnotetext{
${ }^{16}$ Note that since $E_{t-1}\left(\chi_{t}\right)=0$, then $\chi_{t}$ can be viewed as a shock since it is serially uncorrelated and has a zero mean.

${ }^{17}$ Note that since $E_{t}\left(\zeta_{t+1}^{3}\right)$ is a conditional measure it need not be equal to zero, even if $\zeta_{t}$ is normally distributed.
} 
and

$$
\begin{aligned}
\sigma_{i t}^{2}= & \sum_{\tau=1}^{D}\left[r_{i t}(\tau)-a_{r}(\tau)\right]^{2} \\
= & b_{r}^{2} \sum_{\tau=1}^{D} f_{t-1}^{2}(\tau)+c_{r}^{2} \sum_{\tau=1}^{D} \nu_{t-1}^{4}(\tau)+a_{i 0}^{2} \sum_{\tau=1}^{D} \nu_{t}^{2}(\tau)+b_{i 0}^{2} \sum_{\tau=1}^{D} \chi_{t}^{2}(\tau)+\sum_{\tau=1}^{D} \varepsilon_{i t}^{2}(\tau) \\
& +2 b_{r} \sum_{\tau=1}^{D} f_{t-1}(\tau)\left[c_{r} \nu_{t-1}^{2}(\tau)+a_{i o} \nu_{t}(\tau)+b_{i 0} \chi_{t}(\tau)+\varepsilon_{i t}(\tau)\right] \\
& +2 c_{r} \sum_{\tau=1}^{D} \nu_{t-1}^{2}(\tau)\left[a_{i 0} \nu_{t}(\tau)+b_{i 0} \chi_{t}(\tau)+\varepsilon_{i t}(\tau)\right]+2 a_{i 0} \sum_{\tau=1}^{D} \nu_{t}(\tau)\left[b_{i 0} \chi_{t}(\tau)+\varepsilon_{i t}(\tau)\right] \\
& +2 b_{i 0} \sum_{\tau=1}^{D} \chi_{t}(\tau) \varepsilon_{i t}(\tau)+O\left(N^{-1}\right) .
\end{aligned}
$$

It is clear that while individual country returns depend linearly on the first and second order moment innovations, $\nu_{t}$ and $\chi_{t}$, volatility depends on non-linear functions of these innovations and their cross products, and their impacts cannot be identified separately from that of higher order moments of shocks. The presence of these higher order terms, however, induces strong cross sectional dependence (in the sense to be made precise in the following section) in country realized volatilities even if the effects of the growth innovation, $\nu_{t}$, on $r_{i t}$ and $\sigma_{i t}^{2}$ are eliminated. In the next section, we will exploit the difference in the degree of cross sectional dependence of the country output growth rates and realized volatilities, after controlling for the effects of the common growth factor shock, $\nu_{t}$, to identify such innovations from the data, and we will combine all higher order terms in a single common financial shock. In practice, one would expect additional factors such as market imperfections, speculative bubbles and other forms of financial frictions to influence realized equity market volatilities. We therefore view our theoretical model more as a benchmark providing insights for the empirical analysis that follows, rather than a true characterization of the data.

\section{A Static Multi-Country Econometric Framework}

We now build on the theoretical insights that underlie the growth and volatility equations, (1) and (27), and develop a suitable multi-country econometric framework for the empirical analysis of cross country and time variations of the relations between growth and volatility. We begin with a static specification, omitting dynamics and deterministic components to simplify 
the exposition. We also start by positing the following single unobservable common factor representation

$$
\begin{aligned}
v_{i t} & =\lambda_{i} f_{t}+u_{i t}, \\
\Delta y_{i t} & =\gamma_{i} f_{t}+\varepsilon_{i t},
\end{aligned}
$$

for $i=1,2, \ldots, N ; t=1,2, \ldots, T$, where as before $\Delta y_{i t}$ is real GDP growth, ${ }^{18}$ and $v_{i t}=\ln \left(\sigma_{i t}\right)$ is the $\log$ of realized stock market volatility for country $i$ during period $t$ (measured in quarters).

The common factor representation in (28)-(29) is general and motivated by both empirical evidence and standard economic theory. From an empirical perspective, as noted in the introduction, realized equity price volatility and output growth share a large and negative contemporaneous correlation at the country level, for most countries in our sample. This correlation is a robust stylized fact of the data documented also by Baker and Bloom (2013), Carriere-Swallow and Cespedes (2013), and Nakamura et al. (2017). From a theoretical perspective, as shown in the previous section, one can think of $f_{t}$ as a common world growth factor (e.g., technology), which affects all countries GDP growth rates and equity price volatilities contemporaneously, which we will call 'real' factor in the rest of the paper. In view of our theoretical derivations in the previous section, $f_{t}$ could be viewed as a pure 'level' or 'first moment' factor in the sense of Gorodnichenko and Ng (2017).

It is worth noting that without further restrictions on the cross section correlations of $u_{i t}$ and $\varepsilon_{i t}$, it is not possible to be sure about the number of common factors affecting output growth and volatility. In our theoretical derivations, we assume $\varepsilon_{i t}$ to be cross sectionally weakly dependent and derive $u_{i t}$ in terms of level and higher-order moments of $f_{t}$. It follows that most likely $\varepsilon_{i t}$ and $u_{i t}$ will be correlated, and due to the remaining common component affecting $u_{i t}$, it is also likely that $u_{i t}$ will be cross sectionally strongly correlated. This interpretation is also in the spirit of the Arbitrage Pricing Theory of Ross (1976) and Chamberlain and Rothschild (1982) applied to second moments. ${ }^{19}$ In our empirical application we will find that while $f_{t}$ is sufficient to render the cross-country correlations of $\varepsilon_{i t}$ to be weak (required in our theoretical derivations), we need at least one more common factor to span the cross-country correlations of the $u_{i t}$, which we saw in the previous section can be affected by higher order moments of $\Delta y_{i t}$. In the paper, we will

\footnotetext{
${ }^{18}$ We also refer to $\Delta y_{i t}$ as 'output growth' or 'growth' for brevity.

${ }^{19}$ See, for example, Herskovic et al. (2016) and Renault et al. (2016).
} 
refer to this second factor as "financial" and interpret it as a combined higher moment factor.

This econometric specification is not tied to any particular asset price model, but incorporates two practically relevant features: time-varying volatility and heterogeneity in the form of country-specific loadings on the common factors. There are also dynamic features that need to be taken into account that vary across countries due to geographical location, institutions, and history. We will consider heterogeneous dynamics across countries in Section 4.

\subsection{Identifying the Real Factor}

The main idea is to achieve identification of $f_{t}$ and its loadings, $\lambda_{i}$ and $\gamma_{i}$, by placing restrictions on the cross-country correlations of $u_{i t}$ and $\varepsilon_{i t}$, while leaving their within-country correlations unrestricted. To illustrate the strategy, denote global volatility and global GDP growth by $\bar{v}_{\omega, t}$ and $\Delta \bar{y}_{\omega, t}$, respectively, and suppose that they are measured by the weighted cross-section average of country-specific volatility and growth measures:

$$
\begin{aligned}
\bar{v}_{\omega, t} & =\sum_{i=1}^{N} \stackrel{\leftrightarrow}{w}_{i} v_{i t}, \\
\Delta \bar{y}_{\omega, t} & =\sum_{i=1}^{N} w_{i} \Delta y_{i t},
\end{aligned}
$$

where $\left\{\stackrel{\circ}{w}_{i}\right\}$ and $\left\{w_{i}\right\}$ are two sets of aggregation weights, which can be the same or differ for each variable. We make the following assumptions on the common factor, $f_{t}$, and its loadings, $\lambda_{i}$ and $\gamma_{i}$ ), the weights, $\stackrel{\circ}{w}_{i}$ and $w_{i}$, and the country-specific innovations $u_{i t}$ and $\varepsilon_{i t}$ :

Assumption 1 (Common factor and factor loadings) The common unobservable factor $f_{t}$ has zero mean and a finite variance, normalized to one. The factor loadings, $\lambda_{i}$ and $\gamma_{i}$, are distributed independently across $i$ and from the common factors $f_{t}$, for all $i$ and $t$, with non-zero means $\lambda$ and $\gamma(\lambda \neq 0$ and $\gamma \neq 0)$, and satisfy the following conditions, for a finite $N$ and as $N \rightarrow \infty:$

$$
\begin{array}{ccc}
N^{-1} \sum_{i=1}^{N} \lambda_{i}^{2}=\mathcal{O}(1) & \text { and } & N^{-1} \sum_{i=1}^{N} \gamma_{i}^{2}=\mathcal{O}(1), \\
\lambda=\sum_{i=1}^{N} \stackrel{\circ}{w}_{i} \lambda_{i} \neq 0 & \text { and } & \gamma=\sum_{i=1}^{N} w_{i} \gamma_{i} \neq 0 .
\end{array}
$$


Assumption 2 (Weights) Let $\mathbf{w}=\left(w_{1}, w_{2}, \ldots, w_{N}\right)^{\prime}$ and $\stackrel{\circ}{\mathbf{w}}=\left(\stackrel{\circ}{w}_{1}, \stackrel{\circ}{w}_{2}, \ldots, \stackrel{\circ}{w}_{N}\right)^{\prime}$ be the $N \times 1$ vectors of non-stochastic weights with $\sum_{i=1}^{N} w_{i}=1$ and $\sum_{i=1}^{N} \stackrel{\circ}{w}_{i}=1$. These weights need not to be fixed and could be time-varying but predetermined. The growth weights, w, must be granular, in the sense that:

$$
\|\mathbf{w}\|=O\left(N^{-1}\right), \frac{w_{i}}{\|\mathbf{w}\|}=O\left(N^{-1 / 2}\right), \quad \forall i .
$$

The volatility weights $\stackrel{\circ}{\mathbf{w}}$ are also assumed to be granular for ease of exposition, but this assumption could be relaxed.

Assumption 3 (Cross-country correlations) The country-specific innovations, $u_{i t}$ and $\varepsilon_{i t}$, have zero means and finite variances, and are serially uncorrelated, but can be correlated with each other both within and between countries. Furthermore, denoting the variance-covariance matrices of the $N \times 1$ innovation vectors $\varepsilon_{t}=\left(\varepsilon_{1 t}, \varepsilon_{2 t}, \ldots, \varepsilon_{N t}\right)^{\prime}$ and $\mathbf{u}_{t}=\left(u_{1 t}, u_{2 t}, \ldots, u_{N t}\right)^{\prime}$ by $\boldsymbol{\Sigma}_{\varepsilon \varepsilon}=$ $\operatorname{Var}\left(\varepsilon_{t}\right)$ and $\boldsymbol{\Sigma}_{u u}=\operatorname{Var}\left(\mathbf{u}_{t}\right)$, respectively, it is assumed that:

$$
\begin{aligned}
& \varrho_{\max }\left(\boldsymbol{\Sigma}_{u}\right)=\mathcal{O}(N), \\
& \varrho_{\max }\left(\boldsymbol{\Sigma}_{\varepsilon}\right)=O(1) .
\end{aligned}
$$

Assumption 1 is standard in the factor literature (see, for instance, Assumption B in Bai and $\mathrm{Ng}(2002)$ ). It ensures that $f_{t}$ is a strong (or pervasive) factor for both volatility and growth so that it can be estimated consistently either using principal components or by cross-section averages of country-specific observations (see Chudik et al., 2011).

Assumption 2 requires that individual countries' contribution to world growth or world volatility is of order $1 / N$. This is consistent with the notion that, since the 1990 s, when our sample period starts, world growth and world capital markets have become progressively more diversified and integrated as a result of the globalization process.

The first part of Assumption 3 is also standard and leaves the causal relation between the idiosyncratic components, $u_{i t}$ and $\varepsilon_{i t}$, unrestricted. In our model, the correlation between $u_{i t}$ and $\varepsilon_{i t}$ captures any contemporaneous causal relation between country-specific volatility and growth, conditional on $f_{t}$, on which we do not impose any restrictions for the purpose of identifying $f_{t}$.

The second part of Assumption 3 is crucial to identify $f_{t}$ as the following proposition demonstrates. The assumption states that the volatility innovations are strongly correlated across countries, while growth innovations are weakly correlated across countries. Weak cross-country 
correlation in turn means that, asymptotically, as $N$ becomes large, the average pair-wise correlations across countries of growth innovations tends to zero, since the largest eigenvalue of their variance-covariance matrix is bounded in $N .^{20}$ On the other hand, strong cross-country correlation means that the average pair-wise correlation of volatility innovations does not tend to zero because the largest eigenvalue of $\boldsymbol{\Sigma}_{u}$ grows with the size of the cross-section, $N$. As we shall see, this key assumption is in accordance not only with the empirical properties of the data displayed in Figure 2, but also with the properties of the innovations that we obtain from the model estimation. ${ }^{21}$

Proposition 1 (Identification of the real factor) Under Assumptions 1-3, for $N$ sufficiently large, $f_{t}$ can be identified (up to a scalar constant) by $\bar{y}_{\omega, t}=\sum_{i=1}^{N} w_{i} \Delta y_{i t}$.

Proof. Consider the model (28)-(29) for $i=1,2, \ldots, N$. Under Assumptions 1-3, and using the definitions in (30)-(31), the following model for the global variables obtains:

$$
\begin{aligned}
\bar{v}_{\omega, t} & =\lambda f_{t}+\bar{u}_{\omega, t}, \\
\Delta \bar{y}_{\omega, t} & =\gamma f_{t}+\bar{\varepsilon}_{\omega, t},
\end{aligned}
$$

where $\bar{\varepsilon}_{\omega, t}=\stackrel{\circ}{\mathbf{w}}^{\prime} \varepsilon_{t}$, and $\bar{u}_{\omega, t}=\mathbf{w}^{\prime} \mathbf{u}_{t}$. Furthermore:

$$
\operatorname{Var}\left(\bar{\varepsilon}_{\omega, t}\right)=\mathbf{w}^{\prime} \boldsymbol{\Sigma}_{\varepsilon} \mathbf{w} \leq\left(\mathbf{w}^{\prime} \mathbf{w}\right) \varrho_{\max }\left(\boldsymbol{\Sigma}_{\varepsilon}\right)
$$

Thus, under Assumption 3, we have:

$$
\operatorname{Var}\left(\bar{\varepsilon}_{\omega, t}\right)=O\left(\mathbf{w}^{\prime} \mathbf{w}\right)=O\left(N^{-1}\right)
$$

and hence:

$$
\bar{\varepsilon}_{\omega, t}=O_{p}\left(N^{-1 / 2}\right)
$$

Using this in (38), since under Assumption 1, $\gamma \neq 0$, we have:

$$
f_{t}=\gamma^{-1} \Delta \bar{y}_{\omega, t}+O_{p}\left(N^{-1 / 2}\right)
$$

\footnotetext{
${ }^{20}$ See Section 6.2 for a formal discussion of the links between pair-wise correlations and weak and strong crosssectional dependence.

${ }^{21}$ As noted already, patterns of cross-country correlations like the one assumed here, but for consumption growth rather than GDP growth and equity returns rather than equity volatilities, have been documented by Tesar (1995), Colacito and Croce (2011), and Lewis and Liu (2015).
} 
which allows us to recover $f_{t}$ form $\Delta \bar{y}_{\omega, t}$ up to the scalar $1 / \gamma$.

This is a key result in our paper and several remarks are in order:

Remark 1 (Estimation of $f_{t}$ ) As $f_{t}$ is pervasive or strong, we can estimate it with either as the first principal component of the observations $\left\{\Delta y_{i t}\right.$, for $\left.i=1,2, \ldots, N ; t=1,2, \ldots, T\right\}$ or by cross-section averages of $\Delta y_{i t}$, obtaining asymptotically equivalent results.

Indeed, Figure S.2 in the online supplement shows that the first (static) principal component and the cross section average of $\Delta y_{i t}$ provide estimates of $f_{t}$ that are very close, with a correlation of 0.9. In the present context the use of the cross-section-average (CSA) estimator of $f_{t}$ has two advantages. First, it can be directly interpreted as global GDP growth. Second, under Assumptions 1 and 3 the CSA estimator of $f_{t}$ is consistent so long as $N$ is large, whilst the principal component estimator requires both $N$ and $T$ to be large (See section 19.5.1 of Pesaran $(2015))$.

Remark 2 (Principal component on the panel of volatility series) The cross-section average or the principal component of the panel of volatilities series $v_{i t}$ does not identify $f_{t}$.

This is because the volatility innovations, $u_{i t}$, are assumed to be strongly correlated across countries, which in turn means that the largest eigenvalue of $\boldsymbol{\Sigma}_{u}$ will grow with the size of the cross-section (i.e., $\left.\varrho_{\max }\left(\boldsymbol{\Sigma}_{u}\right)=\mathcal{O}(N)\right)$, and $\operatorname{Var}\left(\bar{u}_{\omega, t}\right)=\mathbf{w}^{\prime} \boldsymbol{\Sigma}_{u} \mathbf{w}$ will generally not converge to zero. Under Assumption 3, only the principal component or cross-section average of output growth can be used to identify $f_{t}$.

Remark 3 (Principal component on the combined panel of growth and volatility series) For the same reason, applying principal component analysis to the panel of volatility and growth series does not identify $f_{t}$, either.

Indeed, Figure S.3 in the online supplement shows that the first principal component of the combined panel of volatilities and growth series does not coincides with $f_{t}$ estimated as the cross-section averages of $\Delta y_{i t}$, and its correlation with $\Delta \bar{y}_{\omega, t}$ is -0.43 . The first principal component extracted from the panel of $v_{i t}$ and $\Delta y_{i t}$ captures a linear combination of $f_{t}$ and any additional common factors that exclusively affect the volatility series. 
Before concluding this discussion, it is worth noting that, whilst theoretically we need $N \rightarrow$ $\infty$, in practice $N$ need not be too large for the theory to work reasonably well. The notion of $N \rightarrow \infty$, is best viewed as a counterfactual that considers the extent to which the strength of the connections among units declines with $N$. In our experience, working with many cross-country panels, $N$ around 30 is often sufficiently large.

\subsection{Identifying the Financial Factor}

The main empirical result of the paper does not require explicit identification of the additional strong factors assumed to be exclusive to the volatilities, $v_{i t}$. But doing so permits exploring other properties of the data that underpin the second and the third main empirical results summarized above. With this in mind, recall that, under condition (35) of Assumption 3, the cross-section of volatility innovations, $u_{i t}$, must at least share one additional strong common factor that is not shared by the growth innovations. By assuming, without loss of generality, that the $u_{i t}$ share only one additional strong factor, we can identify it from the data, up to an orthonormal transformation of the factor loadings in the country-specific volatility equations. A formal statement of this result is provided in Proposition 2 below and established under the following assumptions:

Assumption 4 The volatility innovations, $u_{i t}$, in equation (28) can be decomposed into a second, strong, common, unobservable factor, $g_{t}$, and a weak country-specific shock (or idiosyncratic component) $\eta_{i t}$, namely:

$$
u_{i t}=\theta_{i} g_{t}+\eta_{i t}
$$

where $\eta_{i t}$ is cross-sectionally weakly correlated.

The above assumption is compatible with (35) and specifies a one-factor structure for $u_{i t}$. Letting the variance-covariance matrix of the $N \times 1$ vector $\boldsymbol{\eta}_{t}=\left(\eta_{1 t}, \eta_{2 t}, \ldots, \eta_{N t}\right)^{\prime}$ be $\boldsymbol{\Sigma}_{\eta \eta}=$ $\operatorname{Var}\left(\boldsymbol{\eta}_{t}\right)$, under the above assumption, we must also have (as $N \rightarrow \infty$ )

$$
\varrho_{\max }\left(\boldsymbol{\Sigma}_{\eta \eta}\right)=\mathcal{O}(1)
$$

and

$$
N^{-1} \sum_{i=1}^{N} \theta_{i}^{2}=\mathcal{O}(1), \quad \text { and } \quad \theta=\sum_{i=1}^{N} \stackrel{\circ}{w}_{i} \theta_{i} \neq 0
$$


As the next proposition shows, the latter conditions allow us to identify $g_{t}$ from the data, as a liner combination of $\Delta \bar{y}_{\omega, t}$ and $\bar{v}_{\omega, t}$, up to an orthonormal transformation.

Proposition 2 (Identification of the financial factor) Under Assumption 4, the system of equations (28) and (29) can be written as:

$$
\begin{aligned}
v_{i t} & =\lambda_{i} f_{t}+\theta_{i} g_{t}+\eta_{i t}, \\
\Delta y_{i t} & =\gamma_{i} f_{t}+\varepsilon_{i t} .
\end{aligned}
$$

where $i=1,2, \ldots N$, and a second strong factor, $g_{t}$, can be identified up to a liner transformation as $N \rightarrow \infty$. Specifically,

$$
g_{t}=\theta^{-1}\left(\bar{v}_{\omega, t}-\frac{\lambda}{\gamma} \Delta \bar{y}_{\omega, t}\right)+O_{p}\left(N^{-1 / 2}\right)
$$

This result follows from substituting (42) and (43) into (37) and applying the same reasoning applied to $\varepsilon_{i t}$ in Proposition 1 to $\eta_{i t}$.

As we noted earlier, we label $g_{t}$ a 'financial' factor to highlight its role in capturing all higherorder terms in equation (27) once we account for the common real factor shock, $f_{t}$, as well as any bubble component, financial friction, or time-varying risk preference component that might be present in the volatility data. In effect, our identification assumptions distinguish between a first, level factor, $f_{t}$, common to both the growth and volatility series, and everything else common only to the volatility series, lumped together in $g_{t}$. Moreover, we will see below in Proposition 3 that a consistent estimate of $g_{t}$ can be obtained as the residual of a regression of $\bar{v}_{\omega, t}$ on $\Delta \bar{y}_{\omega, t}$. As such, $g_{t}$ captures the orthogonalized effect of the common component to all volatility series once the effect of the factor which is common to both volatility and growth series is filtered out. It is not a pure second-moment factor factor in the sense of Gorodnichenko and Ng (2017), but a composite higher-order factor.

Finally, note that as can be see from (46)-(47), the different patterns of cross-country correlations assumed for volatility and growth innovations implicitly provide restrictions on the factor loadings of the growth equations on the $g_{t}$ factor, and yields country models that have a recursive structure in terms of $f_{t}$ and $g_{t}$, and hence their associated estimates based on cross-section averages. Notice, however, that the identification assumptions made do not require a recursive structure for the country-specific variables, $\Delta y_{i t}$ and $v_{i t}$. 


\subsection{Consistent Estimation of Orthogonalized Real and Financial Factors}

For consistent estimation of the real and financial factors we note that under Assumptions (1)-(4), from equation (42) and (48), we have:

$$
\begin{aligned}
& f_{t}=\alpha_{f} \Delta \bar{y}_{\omega, t}+O_{p}\left(N^{-1 / 2}\right) \\
& g_{t}=\alpha_{1 g} \bar{v}_{\omega, t}-\alpha_{2 g} \Delta \bar{y}_{\omega, t}+O_{p}\left(N^{-1 / 2}\right)
\end{aligned}
$$

for $t=1,2, \ldots T$, where $\alpha_{f}=\gamma^{-1}, \alpha_{1 g}=\theta^{-1}$, and $\alpha_{2 g}=\lambda / \gamma \theta$. It is clear that, for $N$ sufficiently large, $f_{t}$ and $g_{t}$ can be consistently estimated point-wise (at each $t$ ) by a linear combination of $\Delta \bar{y}_{\omega, t}$ and $\bar{v}_{\omega, t}$, without requiring $T$ to be large. Given the recursive structure of the relation between $\left(f_{t}, g_{t}\right)$ and $\left(\Delta \bar{y}_{\omega, t}, \bar{v}_{\omega, t}\right)$, it follows that $f_{t}$ can be estimated up to a scalar constant by average world GDP growth. In contrast, $g_{t}$ is identified as a linear combination of $\Delta \bar{y}_{\omega, t}$ and $\bar{v}_{\omega, t}$ that is unique only up to an orthonormal transformation. It is also evident from (49) and (50) that $g_{t}$ and $f_{t}$ are correlated. The next proposition illustrates that we can proxy $g_{t}$ by setting it equal to the residual of a regression of $\bar{v}_{\omega, t}$ on $\Delta \bar{y}_{\omega, t}$ for all $t$, and thus making it orthogonal to $f_{t}$, without requiring additional economic restrictions.

Proposition 3 (Consistent estimation of orthonormalized factors in the static case) Let $\hat{\zeta}_{t}$ and $\hat{\xi}_{t}$ be consistent, orthonormalized estimators of $f_{t}$ and $g_{t}$, respectively, where $f_{t}$ and $g_{t}$ are defined by (42) and (48). Then, $\hat{\zeta}_{t}$ can be obtained by re-scaling $\Delta \bar{y}_{\omega, t}$ so that its variance is 1 , while $\hat{\xi}_{t}$ can be obtained as the standardized residual of a least squares regression of $\bar{v}_{\omega, t}$ on $\Delta \bar{y}_{\omega, t}$.

Proof. Consider equation (49) and (50) and set the coefficients $\boldsymbol{\alpha}_{g}=\left(\alpha_{1 g}, \alpha_{2 g}\right)^{\prime}$, such that $T^{-1} \sum_{t=1}^{T} \hat{\zeta}_{t} \hat{\xi}_{t}=0$. This yields:

$$
\frac{\hat{\alpha}_{2 g}}{\hat{\alpha}_{1 g}}=\frac{\sum_{t=1}^{T} \Delta \bar{y}_{\omega, t} \bar{v}_{\omega, t}}{\sum_{t=1}^{T} \Delta \bar{y}_{\omega, t}^{2}},
$$

which is the OLS estimate of the coefficient on $\Delta \bar{y}_{\omega, t}$ in a regression of $\bar{v}_{t}$ on $\Delta \bar{y}_{\omega, t}$. Next, set $\alpha_{f}$ and $\alpha_{1 g}$ so that $\zeta_{t}$ and $\xi_{t}$ to have unit in-sample standard deviations. Thus:

$$
\hat{\alpha}_{f}^{2}=\left(\frac{1}{T^{-1} \sum_{t=1}^{T} \Delta \bar{y}_{\omega, t}^{2}}\right)
$$

and:

$$
1=\hat{\alpha}_{1 g}^{2}\left(\frac{\sum_{t=1}^{T} \bar{v}_{\omega, t}^{2}}{T}\right)+\hat{\alpha}_{2 g}^{2}\left(\frac{\sum_{t=1}^{T} \Delta \bar{y}_{\omega, t}^{2}}{T}\right)-2 \hat{\alpha}_{1 g} \hat{\alpha}_{2 g}\left(\frac{\sum_{t=1}^{T} \bar{v}_{\omega, t} \Delta \bar{y}_{\omega, t}}{T}\right)
$$


Hence, we also have:

$$
\hat{\alpha}_{1 g}^{2}=\frac{\left(\frac{\sum_{t=1}^{T} \Delta \bar{y}_{\omega, t}^{2}}{T}\right)}{\left(\frac{\sum_{t=1}^{T} \Delta \bar{y}_{\omega, t}^{2}}{T}\right)\left(\frac{\sum_{t=1}^{T} \bar{v}_{\omega, t}^{2}}{T}\right)-\left(\frac{\sum_{t=1}^{T} \bar{v}_{\omega, t} \Delta \bar{y}_{\omega, t}}{T}\right)^{2}} .
$$

Finally, use $\Delta \bar{y}_{\omega, t}-\Delta \bar{y}_{\omega}$ and $\bar{v}_{\omega, t}-\bar{v}_{\omega}$, where $\Delta \bar{y}_{\omega}=T^{-1} \sum_{t=1}^{T} \Delta \bar{y}_{\omega, t}$ and $\bar{v}_{\omega}=T^{-1} \sum_{t=1}^{T} \bar{v}_{\omega, t}$ in the above formulae to ensure that $\zeta_{t}$ and $\xi_{t}$ have zero means. ${ }^{22}$

Proposition 3 accomplishes two objectives. It derives an observable proxy for $g_{t}$ and it makes sure that the resultant estimator is orthogonal to the proxy for $f_{t}$, which will turn out to be useful when we estimate error variance decompositions and impulse responses. This is achieved simply by choosing coefficients for the linear combination of $\bar{v}_{\omega, t}$ on $\Delta \bar{y}_{\omega, t}$ such that the observable common factors have zero-means, unit variance and are orthogonal to each other. ${ }^{23}$

Remark 4 (World economy as a single-country model versus a multi-country representation) Model (28)-(29) applies to all $N$ countries. Consider a generic country $i$, like for instance the United States, or a closed system like the whole world economy. If we focus on one country in isolation from the rest of the world, or consider a single country global model, we cannot identify the parameters of (28)-(29), even assuming that the innovations $u_{i t}$ and $\varepsilon_{i t}$ are orthogonal to each other and $f_{t}$ is known, unless we exclude $f_{t}$ from one of the two equations.

To see this, note that the covariance matrix of $v_{i t}$ and $\Delta y_{i t}$ is given by:

$$
\Theta_{i}=\left(\begin{array}{cc}
\lambda_{i}^{2}+\sigma_{u, i}^{2} & \lambda_{i} \gamma_{i} \\
\lambda_{i} \gamma_{i} & \gamma_{i}^{2}+\sigma_{\varepsilon, i}^{2}
\end{array}\right)
$$

This provides three independent restrictions, but (28)-(29) contains four free parameters, $\left(\lambda_{i}, \gamma_{i}\right)$, and $\left(\sigma_{u, i}^{2}, \sigma_{\varepsilon, i}^{2}\right)$. Identification of the model parameters requires at least one additional restriction at the country level-for instance, $\lambda_{i}=0$ or $\gamma_{i}=0$, even if it is assumed that innovations $u_{i t}$ and $\varepsilon_{i t}$ are orthogonal to each other.

\footnotetext{
${ }^{22}$ These mean corrections will be applied automatically if intercepts are included in the country-specific models (46) and (47).

${ }^{23}$ In the present static set up, $\zeta_{t}$ corresponds to the growth factor shock $\nu_{t}$ introduced in Section 2; but $\xi_{t}$ need not correspond directly to the growth factor volatility innovations, $\chi_{t}$, also defined in Section 2 . This is because realized volatilities could also be affected by other factors besides growth factor volatility innovations.
} 
To summarize, the key feature of our identification strategy lies in the fact that no restriction is imposed at the individual country level. Restrictions apply only to the cross-section correlations of a large number of countries under consideration. Specifically, we propose a novel approach that exploits the differences in the degree of cross-country correlations for the two series of innovations. The growth innovations are assumed to be cross-sectionally weakly correlated, while the volatility innovations are assumed cross-sectionally strongly correlated. As a result, the effect of country-specific growth innovations on the global variable $\Delta \bar{y}_{\omega, t}$ vanish if $N$ is reasonably large, while the effects of the country-specific volatility innovations on $\bar{v}_{\omega, t}$ do not vanish even if the cross section dimension is large. Under these assumptions the matrix of contemporaneous factor loadings is recursive, which enables us to obtain consistent estimates of the common orthogonal real and financial factors from the data simply by means of OLS, as in a Cholesky decomposition of the variance-covariance matrix of $\Delta \bar{y}_{\omega, t}$ and $\bar{v}_{\omega, t}$, ordering $\Delta \bar{y}_{\omega, t}$ first. Importantly, however, our identification assumptions are not consistent with an alternative ordering of the factors in the Cholesky decomposition where $\bar{v}_{\omega, t}$ is included first. Furthermore, our strategy does not apply to a single country, or to the world economy viewed as a single closed-system. The identification strategy used here is general and can be applied to any panel of time series with the necessary cross-section correlation properties. With this approach, we can identify more factors provided that we consider more cross sections of variables with the appropriate structure of correlation across countries. What is crucial is to adopt a multi-country approach covering a large number of countries, as opposed to considering single country models in isolation.

\section{A Dynamic Multi-Country Heterogeneous Model}

Whilst the static model considered so far is helpful for illustrative purposes, in empirical applications it is important to take dynamics, possibly differing across countries, into account. As we shall see, allowing for dynamics that differ across countries, while requiring additional regularity conditions and derivations, does not alter our main results.

Consider the following first-order dynamic version of the static model (46) and (47):

$$
\begin{aligned}
v_{i t} & =a_{i v}+\phi_{i, 11} v_{i, t-1}+\phi_{i, 12} \Delta y_{i, t-1}+\lambda_{i} f_{t}+\theta_{i} g_{t}+\eta_{i t}, \\
\Delta y_{i t} & =a_{i y}+\phi_{i, 21} v_{i, t-1}+\phi_{i, 22} \Delta y_{i, t-1}+\gamma_{i} f_{t}+\varepsilon_{i t} .
\end{aligned}
$$


In matrix notation we have:

$$
\mathbf{z}_{i t}=\mathbf{a}_{i}+\boldsymbol{\Phi}_{i} \mathbf{z}_{i, t-1}+\boldsymbol{\Gamma}_{i} \mathbf{f}_{t}+\boldsymbol{\vartheta}_{i t}, \quad \text { for } i=1,2, \ldots, N ; t=1,2, \ldots, T,
$$

where $\mathbf{z}_{i t}=\left(v_{i t}, \Delta y_{i t}\right)^{\prime}$ and:

$$
\mathbf{a}_{i}=\left(\begin{array}{c}
a_{i v} \\
a_{i y}
\end{array}\right), \boldsymbol{\Phi}_{i}=\left(\begin{array}{cc}
\phi_{i, 11} & \phi_{i, 12} \\
\phi_{i, 21} & \phi_{i, 22}
\end{array}\right), \boldsymbol{\Gamma}_{i}=\left(\begin{array}{cc}
\lambda_{i} & \theta_{i} \\
\gamma_{i} & 0
\end{array}\right), \mathbf{f}_{t}=\left(\begin{array}{c}
f_{t} \\
g_{t}
\end{array}\right), \boldsymbol{\vartheta}_{i t}=\left(\begin{array}{c}
\eta_{i t} \\
\varepsilon_{i t}
\end{array}\right)
$$

The matrix $\boldsymbol{\Gamma}_{i}$ of contemporaneous factor loadings is assumed to be triangular as per Assumptions 3 and 4 . To accommodate the dynamic nature of the model, we now make the following additional assumptions:

Assumption 5 (Innovations) The country-specific shocks, $\boldsymbol{\vartheta}_{i t}$, are serially uncorrelated (over t), and cross-sectionally weakly correlated (over $i$ ), with zero means, positive definite covariance matrices, $\boldsymbol{\Omega}_{i}$, for $i=1,2, \ldots, N$.

Assumption 6 (Common factors) The $2 \times 1$ vector of unobserved common factors, $\mathbf{f}_{t}=\left(f_{t}, g_{t}\right)^{\prime}$, is covariance stationary with absolute summable autocovariances, and fourth order moments, distributed independently of the country-specific shocks, $\boldsymbol{\vartheta}_{i t^{\prime}}$, for all $i, t$ and $t^{\prime}$.

Assumption 7 (Factor loadings) The factor loadings $\lambda_{i}, \theta_{i}$, and $\gamma_{i}$ (i.e., the non-zero elements of $\left.\boldsymbol{\Gamma}_{i}\right)$ are independently distributed across $i$, and of the common factors, $\mathbf{f}_{t}$, for all $i$ and $t$, with non-zero means $\lambda, \theta$, and $\gamma$, and second-order moments. Furthermore:

$$
\boldsymbol{\Gamma}=\mathbb{E}\left(\boldsymbol{\Gamma}_{i}\right)=\left(\begin{array}{cc}
\lambda & \theta \\
\gamma & 0
\end{array}\right)
$$

Assumption 8 (Coefficients) The constants $\mathbf{a}_{i}$ are bounded, $\boldsymbol{\Phi}_{i}$ and $\boldsymbol{\Gamma}_{i}$ are independently distributed for all $i$, the support of $\varrho\left(\mathbf{\Phi}_{i}\right)$ lies strictly inside the unit circle, for $i=1,2, \ldots, N$, and the inverse of the polynomial $\boldsymbol{\Lambda}(L)=\sum_{\ell=0}^{\infty} \boldsymbol{\Lambda}_{\ell} L^{\ell}$, where $\boldsymbol{\Lambda}_{\ell}=\mathbb{E}\left(\boldsymbol{\Phi}_{i}^{\ell}\right)$ exists and has exponentially decaying coefficients, namely $\left\|\boldsymbol{\Lambda}_{\ell}\right\| \leq K \rho^{\ell}$, where $K$ is a fixed constant and $0<\rho<1$.

These assumptions complement, extend and generalize those made earlier for the static case and allow us to derive consistent estimates of unobservable factors $f_{t}$ and $g_{t}$ in a heterogeneous 
factor-augmented VAR, as summarized in the proposition below. The important additional condition is to control the effects of aggregation of dynamics across the units by requiring that $\Lambda_{\ell}=E\left(\boldsymbol{\Phi}_{i}^{\ell}\right)$ exists and has exponentially decaying coefficients. But it is easily seen that this latter condition holds only if it is further assumed that $\sup _{i} E\left\|\boldsymbol{\Phi}_{i}\right\|<\rho<1$. It is also worth noting that $\boldsymbol{\Gamma}$ defined by (55) is invertible since $\gamma \theta \neq 0$ under Assumption 7 .

Proposition 4 (Consistent estimation of unobservable factors in a dynamic heterogeneous multicountry model) Consider the factor-augmented bivariate VAR models for country $i=1,2, \ldots, N$ given by (54), and suppose that Assumptions 5-8 hold. Then:

$$
\begin{aligned}
& f_{t}=b_{f}+\gamma^{-1} \Delta \bar{y}_{\omega, t}+\sum_{\ell=1}^{\infty} \mathbf{c}_{1, \ell}^{\prime} \overline{\mathbf{z}}_{\omega, t-\ell}+O_{p}\left(N^{-1 / 2}\right) \\
& g_{t}=b_{g}+\theta^{-1}\left(\bar{v}_{\omega, t}-\frac{\lambda}{\gamma} \Delta \bar{y}_{\omega, t}\right)+\sum_{\ell=1}^{\infty} \mathbf{c}_{2, \ell}^{\prime} \overline{\mathbf{z}}_{\omega, t-\ell}+O_{p}\left(N^{-1 / 2}\right),
\end{aligned}
$$

where $b_{f}$ and $b_{g}$ are fixed constants, $\overline{\mathbf{z}}_{\omega, t}=\left(\bar{v}_{\omega, t}, \Delta \bar{y}_{\omega, t}\right),\left\{w_{i}\right.$, for $\left.i=1,2, \ldots, N\right\}$ are fixed weights that satisfy the granularity Assumption 2, and $\mathbf{c}_{1, \ell}^{\prime}$ and $\mathbf{c}_{2, \ell}^{\prime}$ are the first and the second rows of $\mathbf{C}_{\ell}=\boldsymbol{\Gamma}^{-1} \mathbf{B}_{\ell}$, where $\boldsymbol{\Gamma}=\mathbb{E}\left(\boldsymbol{\Gamma}_{i}\right), \mathbf{B}_{\ell}$ is defined by $\boldsymbol{\Lambda}^{-1}(L)=\mathbf{B}_{0}+\mathbf{B}_{1} L+\mathbf{B}_{2} L^{2}+\ldots$, $\boldsymbol{\Lambda}(L)=\sum_{\ell=0}^{\infty} \boldsymbol{\Lambda}_{\ell} L^{\ell}$, and $\boldsymbol{\Lambda}_{\ell}=\mathbb{E}\left(\boldsymbol{\Phi}_{i}^{\ell}\right)$, for all $i$.

Proof. See Appendix A.3.

Notice here that, as shown in Pesaran and Chudik (2014) and Chudik and Pesaran (2015), if slope heterogeneity is not extreme (i.e., if the coefficient matrices $\boldsymbol{\Phi}_{i}$ do not differ too much across $i$ ) and $\mathbf{C}_{\ell}$ decays exponentially in $\ell$, the infinite order distributed lag functions in $\overline{\mathbf{z}}_{\omega, t}$ can be truncated. In practice, Pesaran and Chudik (2014) and Chudik and Pesaran (2015) recommend a lag length $\ell$ equal to $T^{1 / 3}$, where $T$ is the time dimension of the panel.

Notice also that $f_{t}$ and $g_{t}$ are unobservable, while for estimation purposes we need observable factors. However, as $f_{t}$ is identified up a scalar, while $g_{t}$ is identified up to a linear combination of $\bar{v}_{\omega, t}$ and $\Delta \bar{y}_{\omega, t}$, we can continue to proceed similarly to the the case of the static model, as the next proposition illustrates.

Proposition 5 (Consistent estimation of the orthonormalized factors in the dynamic case) Consider $a p^{\text {th }}$ order truncated approximation of the unobservable factors in equation (56) and 
(57) above, and note that in matrix notations we have:

$$
\begin{aligned}
& \mathbf{f}=\Delta \overline{\mathbf{y}}_{\omega}+\overline{\mathbf{Z}}_{\omega} \mathbf{C}_{1}+O_{p}\left(N^{-1 / 2}\right) \\
& \mathbf{g}=\overline{\mathbf{v}}_{\omega}-\lambda \Delta \overline{\mathbf{y}}_{\omega}+\overline{\mathbf{Z}}_{\omega} \mathbf{C}_{2}+O_{p}\left(N^{-1 / 2}\right)
\end{aligned}
$$

where $\mathbf{f}=\left(f_{1}, f_{2}, \ldots, f_{T}\right)^{\prime}, \mathbf{g}=\left(g_{1}, g_{2}, \ldots, g_{T}\right)^{\prime}, \overline{\mathbf{z}}_{\omega}=\left(\boldsymbol{\tau}_{T}, \overline{\mathbf{z}}_{\omega,-1}, \overline{\mathbf{z}}_{\omega,-2}, \ldots, \overline{\mathbf{z}}_{\omega,-p}\right), \overline{\mathbf{z}}_{\omega,-l}=\left(\Delta \overline{\mathbf{y}}_{\omega,-l} \bar{v}_{\omega,-l}\right)$, $\Delta \overline{\mathbf{y}}_{\omega,-l}=\left(\Delta \bar{y}_{\omega, 1-l}, \Delta \bar{y}_{\omega, 2-l}, \ldots, \Delta \bar{y}_{\omega, T-l}\right)^{\prime}, \Delta \overline{\mathbf{y}}_{\omega}=\Delta \overline{\mathbf{y}}_{\omega, 0}, \overline{\mathbf{v}}_{\omega,-l}=\left(\bar{v}_{\omega, 1-l}, \bar{v}_{\omega, 2-l}, \ldots, \bar{v}_{\omega, T-l}\right)^{\prime}$, $\overline{\mathbf{v}}_{\omega}=\overline{\mathbf{v}}_{\omega, 0}$, and $p$ denotes a suitable number of lags (or truncation order). ${ }^{24}$ Consistent estimators of the common shocks, denoted $\boldsymbol{\zeta}$ and $\boldsymbol{\xi}$, can be obtained as residuals from the following OLS regressions:

$$
\begin{aligned}
\Delta \overline{\mathbf{y}}_{\omega} & =\overline{\mathbf{Z}}_{\omega} \hat{\mathbf{C}}_{1}+\hat{\boldsymbol{\zeta}} \\
\overline{\mathbf{v}}_{\omega} & =\hat{\lambda} \hat{\boldsymbol{\zeta}}+\overline{\mathbf{Z}}_{\omega} \hat{\mathbf{C}}_{2}+\hat{\boldsymbol{\xi}}
\end{aligned}
$$

where $\hat{\mathbf{C}}_{1}$ is the OLS estimator of the regression coefficients in the regression of $\Delta \overline{\mathbf{y}}_{\omega}$ on $\overline{\mathbf{Z}}_{\omega}$, and $\hat{\lambda}$ and $\hat{\mathbf{C}}_{2}$ are OLS estimators of the regression coefficients in the regression of $\overline{\mathbf{v}}_{\omega}$ on $\hat{\boldsymbol{\zeta}}$ and $\overline{\mathbf{Z}}_{\omega}$.

Proof. See Appendix A.4.

Remark 5 Since $\hat{\zeta}_{t}$ and $\hat{\xi}_{t}$ are the residuals from regressions of $\Delta \bar{y}_{\omega, t}$ and $\bar{v}_{\omega, t}$ on an intercept and the lagged values $\bar{z}_{\omega, t-1}, \ldots ., \bar{z}_{\omega, t-p}$, it follows that $\hat{\zeta}_{t}$ and $\hat{\xi}_{t}$ will have zero (in-sample) means and, for a sufficiently large value of $p$, will be serially uncorrelated. Therefore, $\hat{\zeta}_{t}$ and $\hat{\xi}_{t}$ can be viewed as estimators of the global innovations (or shocks) to the underlying factors, $f_{t}$ and $g_{t}$. Note however that, unlike the theoretical innovations $\nu_{t}$ and $\chi_{t}$, defined by (22), which could be correlated, the estimators $\hat{\zeta}_{t}$ and $\hat{\xi}_{t}$ are orthogonalized.

Remark 6 In a dynamic setting, the orthogonalized components of $\Delta \bar{y}_{\omega, t}$ and $\bar{v}_{\omega, t}$ ordering $\Delta \bar{y}_{\omega, t}$ first, are not the same as our global shocks $\hat{\zeta}_{t}$ and $\hat{\xi}_{t}$, because this would ignore the contributions of $\overline{\mathbf{z}}_{\omega, t-\ell}$ for $\ell=1,2, \ldots, p$ to the estimation of $f_{t}$ and $g_{t}$. As the factors depend on lagged variables, it is important to make sure that the past values of $\overline{\mathbf{z}}_{\omega, t}$ are filtered out.

Given the orthogonal factor innovations, $\hat{\zeta}_{t}$ and $\hat{\xi}_{t}$, obtained from equation (60) and (61), by

\footnotetext{
${ }^{24}$ The inclusion of $\boldsymbol{\tau}_{T}$ in $\overline{\mathbf{Z}}_{\omega}$ ensures that the filtered factors have zero in-sample means.
} 
substituting them in (54) we can investigate their impact and relative importance for countryspecific volatility and growth based on the following regressions: ${ }^{25}$

$$
\begin{aligned}
v_{i t} & =a_{i v}+\phi_{i, 11} v_{i, t-1}+\phi_{i, 12} \Delta y_{i, t-1}+\beta_{i, 11} \hat{\zeta}_{t}+\beta_{i, 12} \hat{\xi}_{t}+\sum_{\ell=1}^{p} \boldsymbol{\psi}_{v, i \ell}^{\prime} \overline{\mathbf{z}}_{\omega, t-\ell}+\eta_{i t}, \\
\Delta y_{i t} & =a_{i y}+\phi_{i, 21} v_{i, t-1}+\phi_{i, 22} \Delta y_{i, t-1}+\beta_{i, 21} \hat{\zeta}_{t}+\sum_{\ell=1}^{p} \psi_{\Delta y, i \ell}^{\prime} \overline{\mathbf{z}}_{\omega, t-\ell}+\varepsilon_{i t} .
\end{aligned}
$$

These country-specific equations can be estimated consistently by least squares so long as $N$ and $T$ are sufficiently large. As in the static case, large $N$ is required so that the probability order $O_{p}\left(N^{-1 / 2}\right)$ in equations (58) and (59) become negligible. Large $T$ is required to ensure that the dynamics are estimated reasonably accurately. We are now ready to present our empirical results, but before doing so we need to discuss how we measure volatility in our multi-country setting.

\section{Volatility Measurement}

As a proxy for uncertainty we use realized equity price volatility. Realized volatility has been used extensively in the theoretical and empirical finance literature and implicitly assumes that uncertainty and risk can be characterized in terms of probability distributions. ${ }^{26}$ Specifically, we use a measure of quarterly realized volatility based on the summation of daily squared stock price returns. This is a natural application of within-day measures of volatility based on high frequency within-day price changes. ${ }^{27}$

Denote the daily equity price of country $i$, measured at close of day $\tau$ in quarter $t$ as $P_{i t}(\tau)$. The realized volatility for country $i$ in quarter $t$ is computed as:

$$
\sigma_{i t}^{2}=\sum_{\tau=1}^{D_{t}}\left(r_{i t}(\tau)-\bar{r}_{i t}\right)^{2}
$$

where $r_{i t}(\tau)=\Delta \ln P_{i t}(\tau)$, and $\bar{r}_{i t}=D_{t}^{-1} \sum_{\tau=1}^{D_{t}} r_{i t}(\tau)$ is the average daily price changes in the quarter $t$, and $D_{t}$ is the number of trading days in quarter $t$. Note that we have now divided

\footnotetext{
${ }^{25}$ We describe how we can compute the relative importance of these factors for the forecast error variance decomposition of country-specific variables, and the impulse response function of the country specific variables to these shocks in the online supplement to the paper.

${ }^{26}$ It therefore abstracts from Knightian uncertainty, where one cannot attach probabilities to outcomes.

${ }^{27}$ See, for example, Andersen et al. (2001, 2003), Barndorff-Nielsen and Shephard (2002, 2004))
} 
the right hand side of (64) by $D_{t}$ so that our measure of realized volatility is consistent with quarterly GDP growth. Note that, for most time periods, $D_{t}=3 \times 22=66$, which is larger than the number of data points typically used in the construction of daily realized market volatility in the empirical finance literature. ${ }^{28}$ Finally, note that, because variances have rightskewed distributions, but logarithmic variances tend to have near Gaussian distributions, in our empirical application we will be working with the logarithm of realized volatility measures, i.e. $v_{i t}=\log \left(\sigma_{i t}\right)$.

The realized volatility of asset prices is not the only way of measuring 'risk' or 'uncertainty'. If we consider a panel of country equities (e.g., of firms or sectors within a country), a different measure of uncertainty can be computed as the cross-sectional dispersion of equity prices within each country. In Section S1 of the online supplement we show that, under fairly general assumptions, and for $D_{t}$ relatively large (as in our sample), the cross-sectional dispersion of equity returns within country $i$ is closely related to the realized volatility of the country equity returns. So, in our application, we will focus on the realized volatility of country equity indexes. ${ }^{29}$

Realized volatility and cross-sectional dispersion encompass most measures of uncertainty and risk proposed in the literature that could be used to implement our identification strategy. Schwert (1989b), Ramey and Ramey (1995), Bloom (2009), Fernandez-Villaverde et al. (2011) use aggregate time series volatility (i.e., summary measures of dispersion over time of output growth, stock market returns, or interest rates); Leahy and Whited (1996), Campbell et al. (2001), Bloom et al. (2007) and Gilchrist et al. (2013) use dispersion measures at the firmlevel stock market returns; Bloom et al. (2012) use cross-sectional dispersion of plant, firm, and industry profits, stocks, or total factor productivity.

In the finance literature, the focus of the volatility measurement has now shifted to implied volatility measures obtained from option prices, like the US VIX Index (see for example Berger et al., 2017). However, a key input for the implementation of our identification strategy is the availability of country-specific measures of uncertainty for a large number of countries over a long period of time, and implied volatility measures are not yet available for a meaningful number of countries. ${ }^{30}$ The literature has also used uncertainty measures based on expectations

\footnotetext{
${ }^{28}$ In the case of intra-day observations, for example, prices are usually sampled at 10-minutes intervals which yield around 48 intra-daily returns in an 8-hour trading day.

${ }^{29}$ Daily returns are computed abstracting from dividends, which are negligible by comparison to price changes.

${ }^{30}$ Figure S.5 in the supplement plots the US realized volatility measure we constructed with the VIX index during the period over which they overlap and shows that the two measures co-move very closely with a correlation of about 0.9 .
} 
dispersion. ${ }^{31}$ While the measure proposed by Rossi and Sekhposyan (2015) is available for a large number of countries, the time series dimension is unbalanced and often not long enough for our purposes. Finally, model based measures, such as those in Jurado et al. (2015) and Ludvigson et al. (2015) could in principle be computed for all countries in our sample, but the data requirements to construct such measures for many countries over a sufficiently long time period are prohibitive.

\section{Data and Selected Stylized Facts for Volatility and Growth}

This section briefly describes the data set we use in the empirical analysis and reports some stylized facts based on the unconditional moments of the data. Specifically, we consider the degree of persistence in the growth and volatility series, that is relevant for our model specification; and examine the patterns of cross-country correlations, which play an important role in our identification strategy.

The sources of the data and their sampling information are reported in Appendix B. To construct a balanced panel for the largest number of countries for which we have sufficiently long time series, we first collect daily stock prices for 32 advanced and emerging economies from 1979 to 2011 . We then cut the beginning of the sample in 1993, as daily equity price data are not available earlier for two large emerging economies (Brazil and China) and for Peru. Better quality quarterly GDP data for China also became available from 1993. Our results seem to be robust to excluding these three countries and starting the sample in 1988. Moreover, some steps of the empirical analysis, like the estimator of factor innovations $\left(\hat{\zeta}_{t}\right.$ and $\left.\hat{\xi}_{t}\right)$, can be implemented with the unbalanced panel from 1979 without any significant consequence for our main findings.

\subsection{Persistence}

A battery of summary statistics on the realized volatility series and the real GDP series (in levels) supports our model specification in terms of the log-level of realized volatility and the log-differences of real GDP. As Table S.1 in the online supplement shows, the levels of realized volatility, even though persistent, tend to be mean-reverting. Table S.2 in the supplement shows that the first order auto-correlation coefficient for realized volatility is on average about 0.6. Also standard ADF tests reject the null hypothesis of unit roots in the volatility series. In contrast,

\footnotetext{
${ }^{31}$ See, for instance, Zarnowitz and Lambros (1987), Popescu and Smets (2010), Bachmann et al. (2013).
} 
the persistence of real GDP levels is very high (on average around 0.99). Moreover, the null of a unit root for the level of log-GDP cannot be rejected by standard ADF tests for any of the 32 countries in our sample.

\subsection{Cross-country Correlations}

The differential pattern of cross-country correlations of the growth and volatility innovations is crucial for our identification strategy. Here we consider the properties of the observed time series as displayed in Figure 2. In order to gauge the extent to which volatility and growth series co-move across countries, we use two techniques: standard principal component analysis and pair-wise correlation analysis across countries.

In a panel of countries indexed by $i=1,2, \ldots, N$, the average pair-wise correlation of country $i$ in the panel $\left(\bar{\rho}_{i}\right)$ measures the average degree of co-movement of country $i$ with all other countries $j$ (i.e., for all $j \neq i$ ). The average pair-wise correlation across all countries, denoted by $\bar{\rho}_{N}$, is defined as the cross-country average of $\bar{\rho}_{i}$ over $i=1,2, \ldots, N$. This statistics relates to the degree of pervasiveness of the factors, as measured by the factor loadings. To see this, consider equation (29) of our model, $\Delta y_{i t}=\gamma_{i} f_{t}+\varepsilon_{i t}$, where $\operatorname{Var}\left(f_{t}\right)=1$, and $\operatorname{Var}\left(\varepsilon_{i t}\right)=\sigma_{\varepsilon_{i}}^{2}$. The average pair-wise correlation across all countries is given by:

$$
\bar{\rho}_{N}=\frac{2}{N(N-1)} \sum_{i=1}^{N-1} \sum_{j=i+1}^{N} \rho_{i j}=\frac{1}{N(N-1)}\left(\sum_{i=1}^{N} \sum_{j=1}^{N} \rho_{i j}-N\right)
$$

where

$$
\rho_{i j}=\left\{\begin{array}{cc}
\frac{\tilde{\gamma}_{i}}{\sqrt{1+\tilde{\gamma}_{i}^{2}}} \frac{\tilde{\gamma}_{j}}{\sqrt{1+\tilde{\gamma}_{j}^{2}}} & \text { if } i \neq j \\
1 & \text { if } i=j
\end{array}\right.
$$

and $\tilde{\gamma}_{i}=\gamma_{i} / \sigma_{\varepsilon_{i}}$. Substituting the above expression for $\rho_{i j}$ in (65) we have:

$$
\bar{\rho}_{N}=\frac{N}{N-1}\left(\frac{1}{N} \sum_{i=1}^{N} \frac{\tilde{\gamma}_{i}}{\sqrt{1+\tilde{\gamma}_{j}^{2}}}\right)^{2}-\frac{1}{N-1} .
$$

Hence

$$
\bar{\rho}_{N}=O\left(\bar{\gamma}_{N}^{2}\right)
$$

where $\bar{\gamma}_{N}=N^{-1} \sum_{i=1}^{N} \tilde{\gamma}_{i}$ measures the degree of pervasiveness of the factor. 
The attraction of the average pair-wise correlation, $\bar{\rho}_{N}$, lies in the fact that it applies to multi-factor processes, and unlike factor analysis does not require the factors to be strong. In fact, the average pair-wise correlation, $\bar{\rho}_{N}$, tends to be a strictly positive number if $\Delta y_{i t}$ contains at least one strong factor, otherwise it tends to zero as $N \rightarrow \infty$. Therefore, non-zero estimates of $\bar{\rho}_{N}$ are suggestive of strong cross-sectional dependence. ${ }^{32}$ For completeness, and to show that our analysis is robust to using an alternative methodology, in what follows, we also use standard principal component analysis. ${ }^{33}$

Country-specific average pair-wise correlations of volatility and GDP growth are reported in Figure 2. Recall that the average pair-wise correlation across all countries for the realized volatility series is 0.56 . In contrast, the average pair-wise correlation across all countries for the growth series at 0.27 is much smaller. As we can see, the pair-wise correlations of volatility and growth have a similar values for different countries, but there is a clear difference between the two variables. This suggests that both variables may share at least one strong common factor, even though volatilities seem to co-move more across countries than the GDP growth rates.

Principal component analysis yields similar results. The first principal component in our panel of realized volatility series explains 65 percent of the total variation in the log-level of volatility, whilst the first principal component of the growth series accounts for only around 30 percent of total cross-country variations. Thus, both in the case of the pair-wise correlation and principal component analysis, the results point to a much higher degree of cross-country comovements for the volatility series than for the growth series. As we will see, these differences are even more pronounced in the case of the estimated innovations series obtained using equations (62) and (63).

\section{Estimated Common and Country-specific Components}

The preliminary analysis above is compatible with the common factor model proposed in the paper, suggesting a stronger degree of cross-country co-movements for volatility series as compared to the growth series. The summary statistics reported also support the model specification in terms of log-level of volatility and log-difference of growth. Now we will use our multi-country factor-augmented VAR model, (62) and (63), to interpret the observed negative association

\footnotetext{
${ }^{32}$ Formal tests of cross-sectional dependence based on estimates of $\bar{\rho}_{N}$ are discussed in Pesaran (2015) and reported, for our panel of countries, in the next section.

${ }^{33}$ See also Chapter 29 in Pesaran (2015).
} 
between volatility and growth.

We begin by estimating the global factor innovations, $\hat{\zeta}_{t}$ and $\hat{\xi}_{t}$, using (60) and (61). We then estimate country-specific VAR models conditional on these innovations and obtain estimates of the country-specific growth and volatility innovations, $\varepsilon_{i t}$ and $\eta_{i t}$. The necessary computations are carried out by applying OLS to (62)-(63) for each $i$ separately. Finally, we compute and report conditional pair-wise correlations across countries for country-specific volatility and growth innovations to evaluate our identification assumptions, and within-country correlations between volatility and growth innovations to assess the model's ability to capture the countercyclical nature of realized volatility. It is important to note that we will also estimate country-specific volatility innovations, $u_{i t}$, by conditioning only on $\hat{\zeta}_{t}$ rather than both on $\hat{\zeta}_{t}$ and $\hat{\xi}_{t}$ in (62)-(63). Thus, we will present results for estimated values of both $\eta_{i t}$ and $u_{i t}$ as the latter are derived under weaker assumptions.

\subsection{Estimated Global Real and Financial Shocks}

The global shocks, $\hat{\zeta}_{t}$ and $\hat{\xi}_{t}$, are recovered from the OLS estimation of (60) and (61). Figure 3 plots them when estimated using the unbalanced panel from 1979 (thin lines with asterisks), and when we use the balanced panel from 1993 (thick solid lines), so as to better illustrate their time profiles. The figure also reports one-standard deviation bands for the shocks. Note that the shocks are standardized and have zero means and unit in-sample variances. They are also serially uncorrelated and orthogonal to each other.

The figure shows that the largest common real shock was after the second oil shock in 1979, and during the fourth quarter of 2008 after the Lehman's collapse. Also note that, after a very large positive shock in the second quarter of 2009, the size of the subsequent impulses declines persistently, which is consistent with the anemic global recovery experienced in the aftermath of the global financial crisis. The sizes of largest absolute values of common financial shocks, $\hat{\xi}_{t}$ coincide with the 1987 stock market crash and the 2008 Lehman's collapse. Our estimates of global financial shocks co-move positively, but not closely, with the US measures of financial volatility, risk and investor sentiment. In general, $\hat{\xi}_{t}$ series is more volatile and by construction less persistent as compared to the these (raw) US measures. The correlation of $\hat{\xi}_{t}$ with the US Baa-Treasury credit spread and Excess Bond Premium of Gilchrist and Zakrajsek (2012) is 0.20 
Figure 3 Estimated Common Real $\left(\hat{\zeta}_{t}\right)$ and Financial $\left(\hat{\xi}_{t}\right)$ Shocks

Panel A: Common real shock $\left(\hat{\zeta}_{t}\right)$

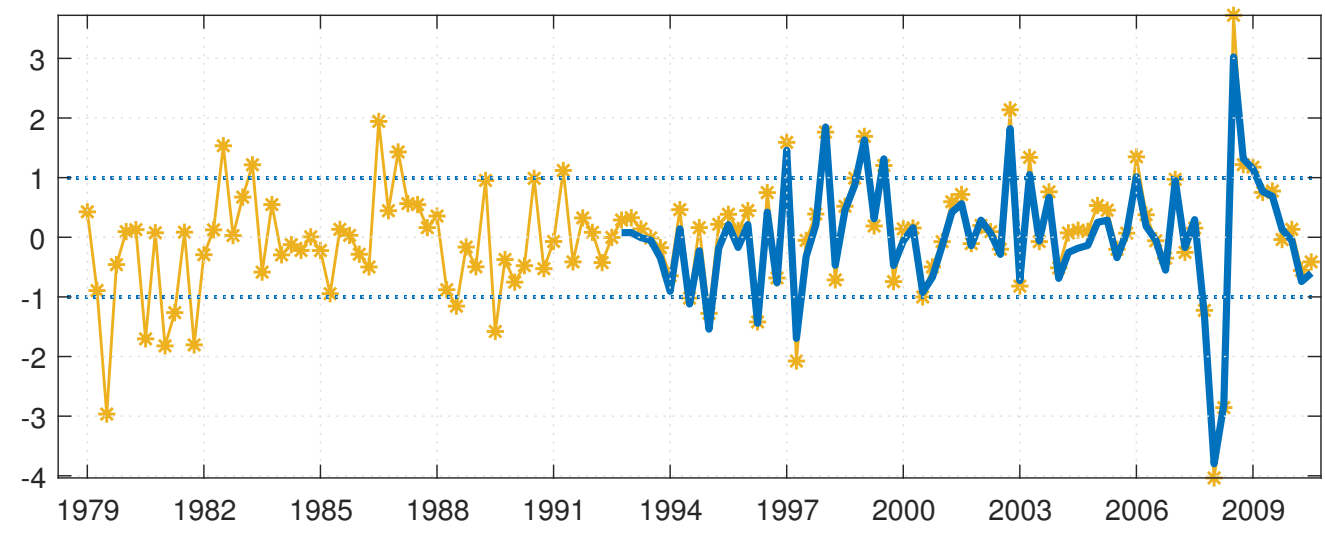

Panel B: Common financial shock $\left(\hat{\xi}_{t}\right)$

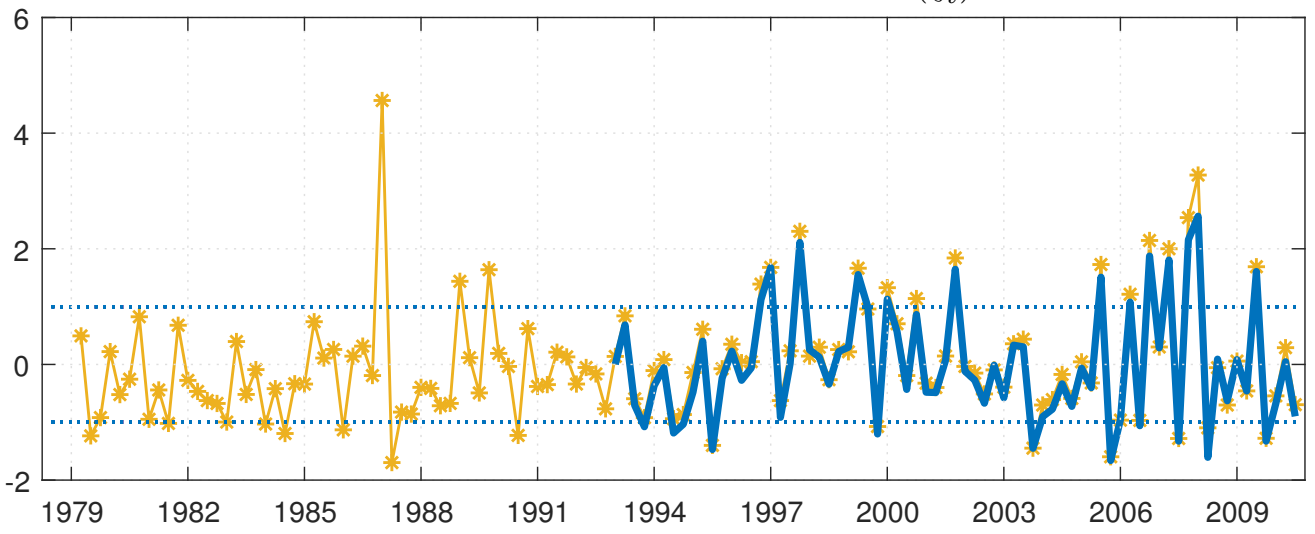

Note. The shocks $\hat{\zeta}_{t}$ and $\hat{\xi}_{t}$ are computed using (60) and (61), with one lag of $\mathbf{z}_{i t}$, using the full unbalanced sample 1979:Q2-2011:Q2 (thin lines with asterisks) and the shorter balanced sample 1993:Q1-2011:Q2 (thick solid lines). The shocks are standardized and the dotted lines are the one-standard deviation bands around the zero mean.

and 0.35 , respectively. ${ }^{34}$ Its correlation with the index of financial volatility of Ludvigson et al. (2015) is about 0.4, and with the US VIX index is 0.5. This evidence suggests that our series of global financial shocks has distinct information content as compared to a number of related US-specific measures.

In the online supplement to the paper, we compare the above results with those obtained using the principal components. Specifically, we first show that, when we recover $\hat{\zeta}_{t}$ using principal components applied to the panel of growth rates, $\Delta y_{i t}$, we obtain virtually the same results, as expected and highlighted in Remark 1 above. Second, we show that when we run

\footnotetext{
${ }^{34}$ The Baa-Treasury credit spread is computed as the difference between yields on long-term Baa-rated industrial bonds and comparable maturity Treasury securities.
} 
principal component analysis on the panel of volatilities, $\left(v_{i t}\right)$, or volatilities and growth rates, $\left(\Delta y_{i t}^{\prime}, v_{i t}^{\prime}\right)^{\prime}$, we do not recover $\hat{\zeta}_{t}$, as also stated earlier in Remarks 2 and 3. Finally, in the online supplement to the paper, we show that one can approximately recover $\hat{\zeta}_{t}$ and $\hat{\xi}_{t}$ by applying the principal component analysis in a recursive manner, provided the recursive estimation is carried out with $\Delta y_{i t}$ first, followed by $v_{i t}$, and not vice versa.

\subsection{Cross-country Correlations of Volatility and Growth Innovations}

Although the restrictions behind our identification assumptions cannot be formally tested, our multi-country approach permits us to investigate the extent to which the implications of the identified model are in line with the identification restrictions made. ${ }^{35}$ To this end, we explore the cross-country correlations of the estimated residuals from the dynamic regressions (62) and (63), with and without conditioning on the financial shocks series, $\hat{\xi}_{t} \cdot{ }^{36}$

Panel A of Figure 4 plots, for each country in our sample, the average pair-wise correlation of the volatility innovations $\left(\hat{u}_{i t}\right)$ and the growth innovations $\left(\hat{\varepsilon}_{i t}\right)$, when we condition only on $\hat{\zeta}_{t}$ in model (62)-(63). Panel B reports the same statistics when we condition on both $\hat{\zeta}_{t}$ and $\hat{\xi}_{t}$ in model (62)-(63). ${ }^{37}$ The statistics reported are the same as in Figure 2. The blue (darker) bars are the average pair-wise correlations of the country-specific growth innovations $\left(\hat{\varepsilon}_{i t}\right)$. The yellow (lighter) bars are for the country-specific volatility innovations ( $\hat{u}_{i t}$ and $\hat{\eta}_{i t}$, respectively).

Panel A of Figure 4 shows that, if we condition only on $\hat{\zeta}_{t}$ in (62)-(63), the volatility innovations display average pair-wise correlations comparable to those of the data reported for all countries in Figure 2. In contrast, the pair-wise correlations of the growth innovations are negligible, with an average across all countries of $0.03 .{ }^{38}$ Panel B of Figure 4 also shows that, if we condition on both $\hat{\zeta}_{t}$ and $\hat{\xi}_{t}$, the cross-country correlations of the volatility innovations are now negligible, as in the case of the growth innovations, with an average pair-wise correlation across all countries equal to 0.02. For instance, in the specific case of the US, the average pair-wise correlation of the volatility innovations is equal to 0.6 conditioning on $\hat{\zeta}_{t}$ alone. But it drops to -0.05 if we condition on both factor innovations. By comparison, the US average pair-wise

\footnotetext{
${ }^{35}$ Note that we can estimate $\zeta_{t}$ and $\xi_{t}$ consistently by means of the OLS regressions (60) and (61) only under the identification assumptions made. As a result, whilst we can directly estimate pair-wise correlations of volatility and growth series, we can not examine cross-country pair-wise correlations of their innovations without imposing these identification conditions.

${ }^{36}$ Recall that, in this case, we run OLS on (62) without conditioning on $\hat{\xi}_{t}$ in the regression.

${ }^{37}$ The same growth innovations are obtained in the two exercises.

${ }^{38}$ Notable exceptions are China and India.
} 


\section{Figure 4 Cross-COUNTRY CORRELATion of COUNTRY-SPECIFIC Volatility and Growth InNOVATions}
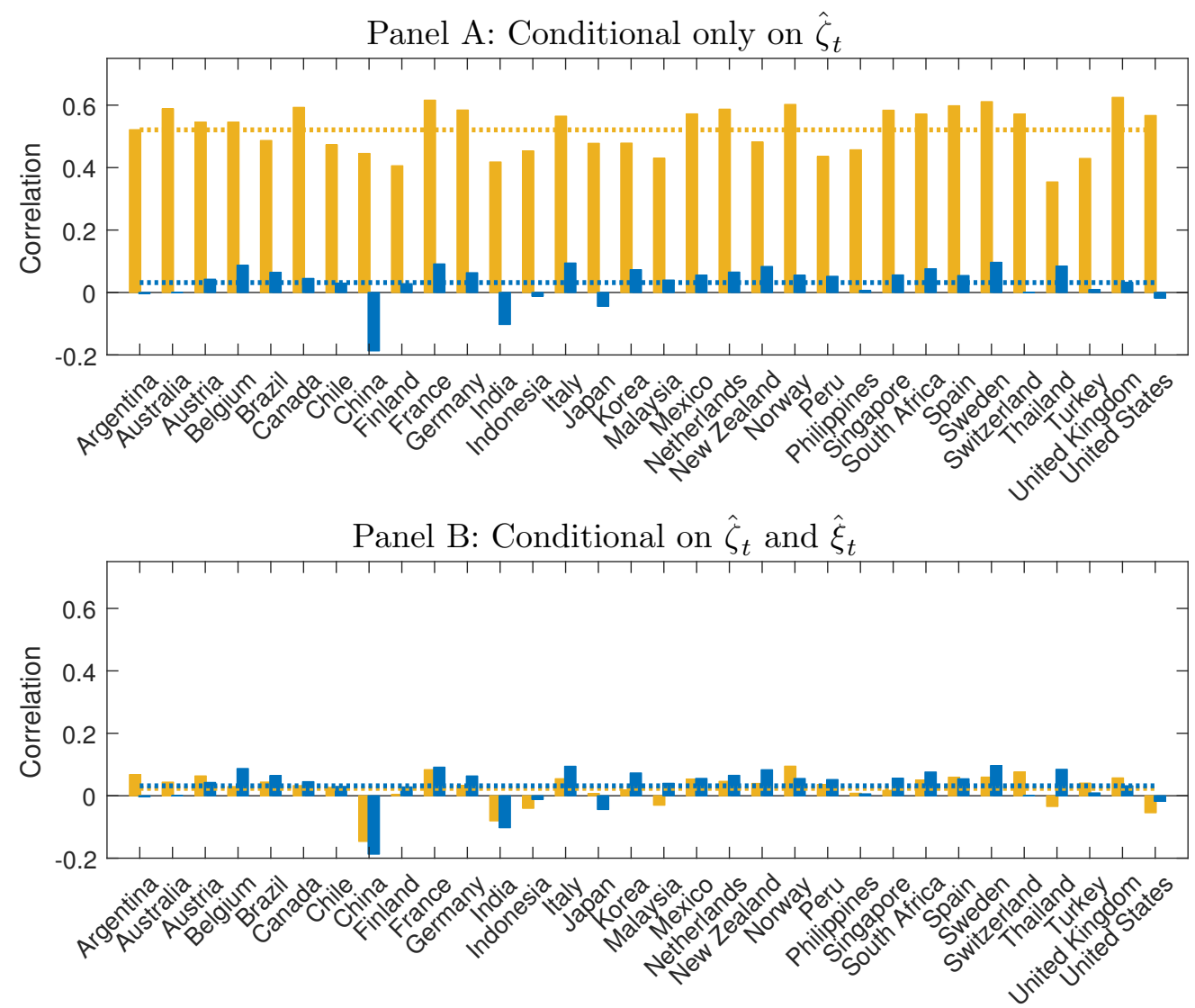

Note. Country-specific average pair-wise correlation of volatility (yellow, lighter bars) and GDP growth (blue, darker bars) innovations conditional on $\hat{\zeta}_{t}$ only (Panel A) and on $\hat{\zeta}_{t}$ and $\hat{\xi}_{t}$ (Panel B). The volatility measures are based on (64). The dotted lines are the averages across all countries, equal to 0.52 and 0.03 for volatility and growth in Panel A; and equal to 0.02 and 0.03 for volatility and GDP growth in Panel B, respectively. Sample period: 1993:Q1-2011:Q2.

correlation of the growth innovations is -0.02 .

Figure 4, therefore, illustrates that after conditioning on $\hat{\zeta}_{t}$ - which is common to both growth and volatility series - not much commonality is left in the case of growth innovations, but the volatility innovations share additional strong factors. Moreover, after conditioning on both $\hat{\zeta}_{t}$ and $\hat{\xi}_{t}$, the volatility innovations also appear weakly correlated because of the near-zero average pair-wise correlation across all countries, thus suggesting that only two common shocks are necessary to span their correlations across-countries as we assume. It is, therefore, interesting to test whether the two sets of innovations also satisfy a formal definition of weak and strong dependence, as we assumed deriving them. 
To test for weak and strong cross-section dependence, we estimate the cross-sectional dependence (CD) test statistic of Pesaran (2015) and the exponent of cross sectional dependence $(\alpha)$ proposed in Bailey et al. (2016). The CD statistic is normally distributed with zero-mean and unit-variance under the null of zero average pair-wise correlations. So, the critical value is around 2. When the null is rejected, Bailey et al. (2016) suggest estimating the strength of the cross-section dependence with an exponent, denoted $\alpha$ in the range $(1 / 2,1]$, with unity giving the maximum degree of cross dependence. Any value above 1/2 and below 1, but significantly different from 1 , suggests weak dependence. ${ }^{39}$ So, in what follows, we present estimates of $\alpha$ for the volatility and the growth innovations, together with their confidence intervals. For comparison, we also report the same estimates for the (raw) growth $\left(\Delta y_{i t}\right)$ and volatility $\left(v_{i t}\right)$ series.

Table 1 Testing for the Strength of Cross-Sectional Dependence

\begin{tabular}{|c|c|c|c|c|}
\hline & $C D$ & Lower $5 \%$ & $\hat{\alpha}$ & Upper $95 \%$ \\
\hline \multicolumn{5}{|l|}{ Data } \\
\hline$v_{i t}$ & 57.00 & 0.96 & 1.00 & 1.05 \\
\hline$\Delta y_{i t}$ & 29.64 & 0.83 & 1.00 & 1.17 \\
\hline \multicolumn{5}{|c|}{ Innovations (conditional on $\hat{\zeta}_{t}$ ) } \\
\hline$\hat{u}_{i t}$ & 57.31 & 0.95 & 1.00 & 1.05 \\
\hline$\hat{\epsilon}_{i t}$ & 5.07 & 0.75 & 0.80 & 0.86 \\
\hline \multicolumn{5}{|c|}{ Innovations (conditional on $\hat{\zeta}_{t}$ and $\hat{\xi}_{t}$ ) } \\
\hline$\hat{\eta}_{i t}$ & 2.13 & 0.57 & 0.65 & 0.73 \\
\hline
\end{tabular}

Note. $C D$ is the cross-sectional dependence test statistic of Pesaran (2015). $\hat{\alpha}$ is the estimate of the exponent of cross-sectional dependence as in Bailey et al. (2016), together with its 90-percent confidence interval ('Lower 5\%' and 'Upper95\%').

The results are summarized in Table 1 and are in accordance with the identification assumptions made. The CD test statistic for the growth series is 29.64, with the associated $\alpha$ exponent estimated at 1.00. The CD statistic for the volatility series is even higher at 57.00 with an estimated $\alpha$ of 1.00. The CD statistics and the estimates of the $\alpha$ exponent confirm with a high degree of confidence that both series are cross-sectionally strongly correlated, containing at least one strong common factor. Conditional only on $\hat{\zeta}_{t}$, the CD statistic for the countryspecific growth innovations $\left(\hat{\varepsilon}_{i t}\right)$ drops to 5.07 , close to its critical value under the null of zero

\footnotetext{
${ }^{39}$ When estimating $\alpha$ one also needs to take into account the sampling uncertainty which depends on the relative magnitude of $N$ and $T$, and the null of weak cross dependence depends on the relative rates of increase of $N$ and $T$.
} 
average pair-wise correlations, with its exponent of cross-sectional dependence estimated to be 0.80 , and is significantly below 1 . In sharp contrast, the CD statistic for the country-specific volatility innovations $\left(\hat{u}_{i t}\right)$ remains close to that of the raw volatility series at 57.31 with an estimated $\alpha$ close to unity. However, when we condition on both $\hat{\zeta}_{t}$ and $\hat{\xi}_{t}$, the CD statistic for the volatility innovations $\left(\hat{\eta}_{i t}\right)$ also falls to 2.13 , with an estimated $\alpha$ of 0.65 and a 95 percent confidence interval of $[0.57,0.73]$, while the $\mathrm{CD}$ statistic and $\alpha$ are the same as before for the growth innovations $\left(\hat{\varepsilon}_{i t}\right)$. The battery of test statistics in Table 1, therefore, accord well with the assumptions made that the volatility innovation share at least one more (Assumption 3), and only one more (Assumption 4) strong factor, than the growth innovations.

\subsection{Country-specific Correlations Between Volatility and Growth Innova- tions}

We have shown already that realized equity price volatility is countercyclical in almost all countries in our sample. Figure 5 suggests that this association is almost entirely accounted for by the global output growth innovations, $\hat{\zeta}_{t}$. Figure 5 compares unconditional and conditional contemporaneous correlations between volatility and growth. For ease of comparison, Panel A displays again the correlations of volatility and growth series reported in Figure 1. Panel B shows the correlation between $\hat{u}_{i t}$ and $\hat{\varepsilon}_{i t}$ conditional on $\hat{\zeta}_{t}$ only, as in (63)-(62). Panel C, reports the correlation between $\hat{\eta}_{i t}$ and $\hat{\varepsilon}_{i t}$, conditional on both $\hat{\zeta}_{t}$ and $\hat{\xi}_{t}$.

Figure 5 shows that $\hat{\zeta}_{t}$ can account for most of the unconditional association between volatility and growth at quarterly frequency reported in Panel A. ${ }^{40}$ Conditioning on $\hat{\zeta}_{t}$, the correlation between volatility and growth innovations weakens substantially for all countries and it is no longer statistically significant in all but two cases, which happen to be very volatile emerging economies. In the case of the US, for instance, the conditional correlation does not vanish, but drops to less than half its unconditional value and is not statistically significant. Panel $\mathrm{C}$ of Figure 5 shows that conditioning explicitly on $\hat{\xi}_{t}$ does not alter these results. This is intuitive, as $\hat{\xi}_{t}$ is common only to the volatility series.

These results suggest that volatility and growth share an important common component at quarterly frequency, and that conditioning on $\hat{\zeta}_{t}$ captures most of this dependence. This implies that some of the explanatory power attributed to uncertainty shocks in empirical studies of

\footnotetext{
${ }^{40}$ Recall that this result does not depend on the granularity of the volatility weights in Assumption 2 and on Assumption 4.
} 
Figure 5 Country-Specific Correlations Between Volatility and Growth INNOVATIONS

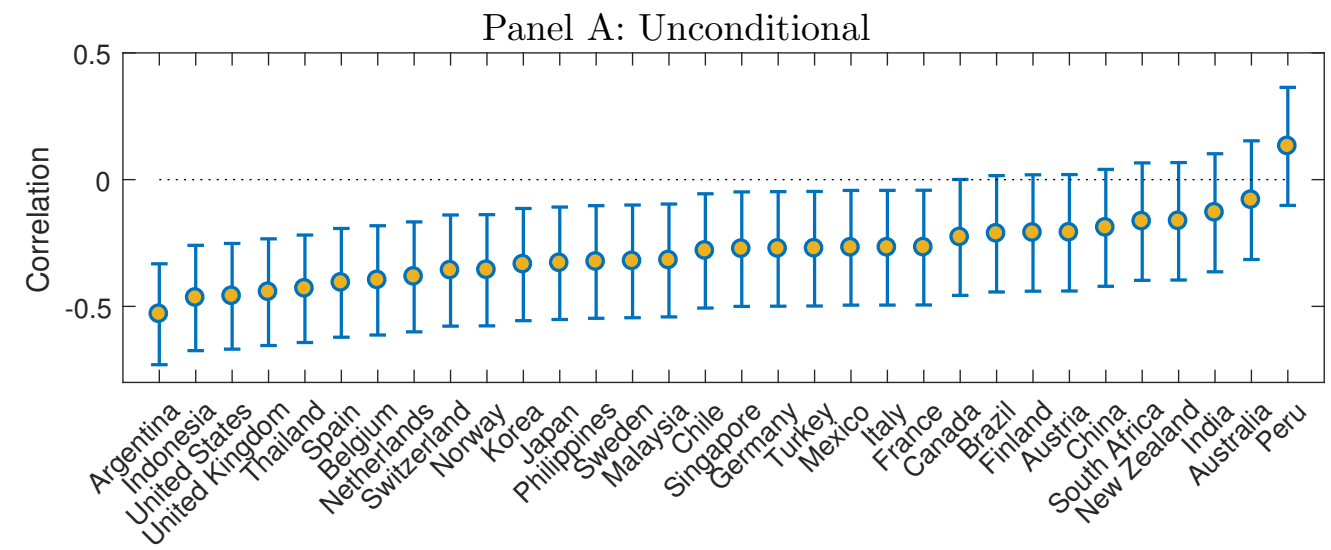

Panel B: Conditional on $\hat{\zeta}_{t}$ only

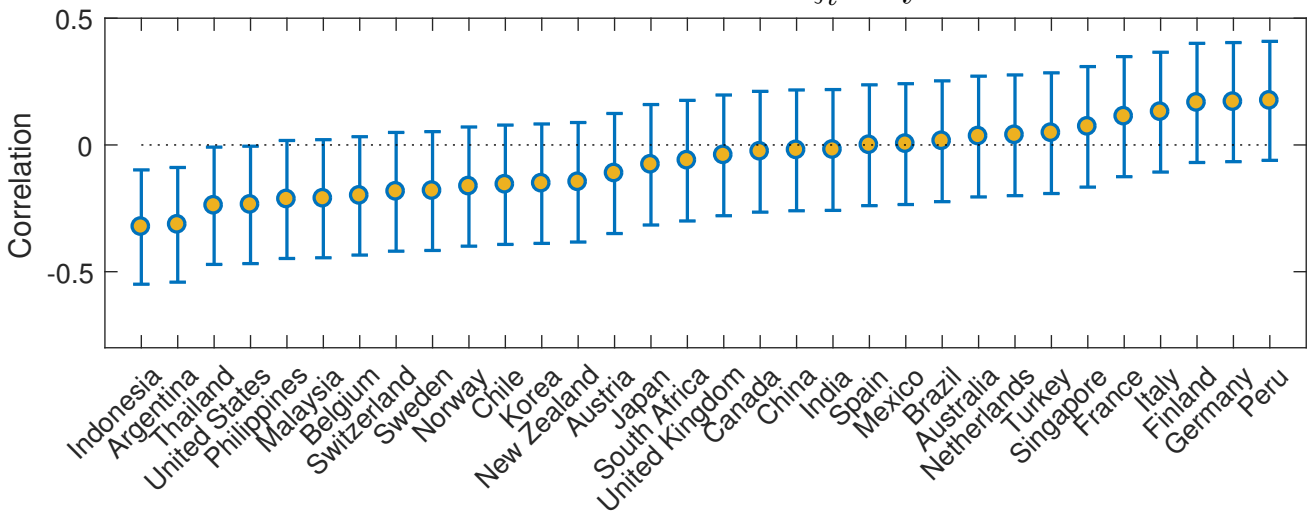

Panel C: Conditional on $\hat{\zeta}_{t}$ and $\hat{\xi}_{t}$

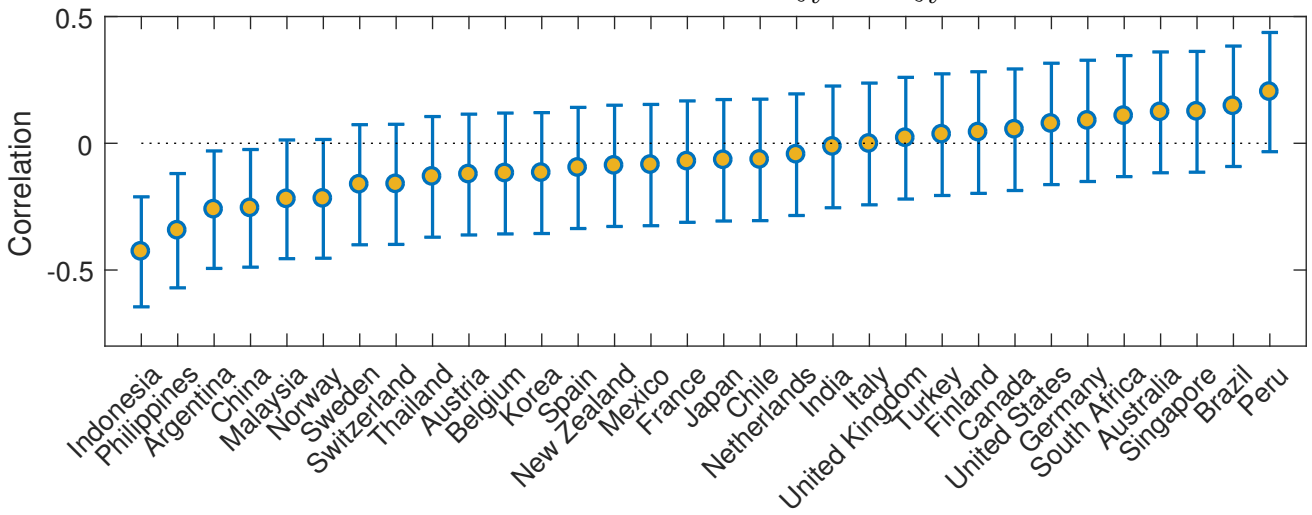

Note. Panel A displays the unconditional correlations plotted as in Figure 1. Panel B reports the volatility innovations $\left(\hat{u}_{i t}\right)$ captured as residuals from equation (62) without including the $\hat{\xi}_{t}$ shocks; Panel C reports the volatility innovations $\left(\hat{\eta}_{i t}\right)$ captured as residuals from equation (62). In both Panel B and Panel C, the growth innovations $\left(\hat{\varepsilon}_{i t}\right)$ are the residuals of equation (63). The dots represent the contemporaneous correlations. The lines represent 95\% confidence intervals. Sample period: 1993:Q1-2011:Q2. 
individual countries, considered in isolation from the rest of the world, might be due to omitting such a common factor from the analysis.

How can we interpret this evidence? While we are agnostic on which specific structural model may or may not generate the pattern of cross-country correlations that we have assumed in our identification strategy and documented above, given our theoretical model, it is reasonable to interpret the common growth innovations, $\hat{\zeta}_{t}$, as reflecting variations in the global risk free rate. Specifically, in Section 2.1 and 2.2, using a simple multi-country model, we showed that expected world growth is tied to the world risk-free rate, and changes in expected world growth can affect both country volatilities and country growth rates at the same time. Lower expected world growth means a lower world risk-free rate, and hence higher country risk premium and country volatility, but also lower country growth. Our real common factor, therefore, drives both variables.

Note, however, that the evidence reported above does not imply that changes in volatility over time are mostly driven by $\hat{\zeta}_{t}$. That is, while these shocks can account for most of the contemporaneous co-movement between volatility and growth, they do not necessarily explain a significant share of the observed time variations in global innovations to volatility. Indeed, as we will see in the next section, $\hat{\zeta}_{t}$ explains a relatively small share of the variation of volatility over time, with a much larger share explained by $\hat{\xi}_{t}$.

\section{Volatility and Growth Forecast Error Variance Decomposi- tions}

Forecast error variance decompositions are routinely used to quantify the importance of a given shock for the time-variation of the endogenous variables at different time horizons, relative to other shocks in the model. Our factor augmented multi-country VAR model can be readily used to decompose the forecast error variance of country volatility and growth in terms of the common shocks, $\hat{\zeta}_{t}$ and $\hat{\xi}_{t}$, as well as the 64 vector of country-specific shocks, $\hat{\eta}_{i t}$ and $\hat{\varepsilon}_{i t}$ for $i=1,2, \ldots ., 32$. While the global real and financial shocks, $\hat{\zeta}_{t}$ and $\hat{\xi}_{t}$, are orthogonal to the country-specific shocks and to each other by construction, the country-specific shocks $\hat{\eta}_{i t}$ and $\hat{\varepsilon}_{i t}$ are left unrestricted, and can be correlated, both within and between countries, even conditional on $\hat{\zeta}_{t}$ and $\hat{\xi}_{t}$. In order to compute and interpret forecast error variance decompositions, we therefore have to deal 
with this source of within country interdependence.

Consider first the correlation between volatility and growth innovations within each country. We saw in Figure 5 that the contemporaneous within-country correlation between $\hat{\eta}_{i t}$ and $\hat{\varepsilon}_{i t}$ is very small and not statistically significant for all but two emerging market economies, once we condition on the global shocks $\hat{\zeta}_{t}$ and $\hat{\xi}_{t}$. Nonetheless, even assuming the estimated reduced form covariance matrix were truly diagonal, this would not imply that innovations $\hat{\eta}_{i t}$ and $\hat{\varepsilon}_{i t}$ can be interpreted as 'structural' country-specific volatility and growth shocks. As it is well known there always exists an orthonormal transformation of $\hat{\eta}_{i t}$ and $\hat{\varepsilon}_{i t}$ that lead to the same forecast error variance decomposition.

It is, therefore, important that the $64 \times 64$ matrix of correlations among all 32 countries and both variables is considered in a full multi-country set up. Our results show that, conditional on both real and financial common shocks, $\hat{\zeta}_{t}$ and $\hat{\xi}_{t}$, the country-specific innovations $\hat{\varepsilon}_{i t}$ and $\hat{\eta}_{i t}$ are weakly correlated across countries (Figure 4). The average pair-wise correlations of volatility and growth is negligible, and even in the case of China and India they were well below 0.2 . As we discussed above, weak cross-sectional dependence means that, as $N$ grows, the overall average pair-wise correlation tends to zero. This further means that, while some pairs of correlations can be different from zero, not all pairs can be so. In practice, this means that most correlation pairs will be very small and the overall covariance matrix must be sparse.

We exploit the sparsity of the correlation matrix of country-specific shocks by making alternative assumptions regarding the causal relations between the country-specific innovations $\hat{\eta}_{i t}$ and $\hat{\varepsilon}_{i t}$, and show that the inference we draw is reasonably robust to different estimates of the country-specific error correlation matrix. As a first approximation, we assume that the only source of interdependence among all growth and volatility series are the global real and financial shocks $\hat{\zeta}_{t}$ and $\hat{\xi}_{t}$. This implies assuming that country-specific volatility and growth shocks have no contemporaneous impact on growth or volatility series within and across countries. Despite its apparent severity, this assumption seems justified by our empirical finding that there exist very limited conditional within-country correlations and weak cross-country correlations as summarized above.

We then check the robustness of the results from this 'benchmark' scenario, by comparing them with those obtained under weaker assumptions. While maintaining the assumption of zero conditional correlations between countries, we assume that country-specific volatility shocks can 
have a contemporaneous causal impact on growth variables but not vice-versa, in line with much of the existing empirical literature as reviewed in the Introduction. This is done by allowing for a block-diagonal error covariance matrix in the full multi-country model, in which the only nonzero off-diagonal elements are the estimated covariances between volatility and growth errors of each country block. These within-country blocks are factorized with a Cholesky decomposition, ordering volatility before growth.

Finally, as a third possibility we refrain altogether from interpreting country-specific volatility and growth shocks as structural, and make use of a general unrestricted error covariance matrix subject to the sparsity condition, both within and across countries and compute the generalized forecast error variance decompositions (GFEVD) of Pesaran and Shin (1998), rather than orthogonal forecast error variance decompositions that require Cholesky ordering of the shocks. However, before computing GFEVDs, we use the regularized multiple testing threshold estimator of the error covariance matrix proposed by Bailey et al. (2017) and described in more detail below, to obtain a consistent estimator of the $64 \times 64$ error covariance matrix for the full multi-country model. This regularized estimator exploits the sparsity of the underlying error covariance matrix.

In what follows we report results for these three alternative specifications of the covariance matrix of the innovations $\hat{\varepsilon}_{i t}$ and $\hat{\eta}_{i t}$. As we wish to quantify the relative importance of both the real and the financial common shocks, all results are based on (62)-(63) that include both $\hat{\zeta}_{t}$ and $\hat{\xi}_{t}$. Specifically, Figure 6 reports the forecast error variance decompositions (FEVDs) obtained assuming the $64 \times 64$ error covariance matrix is diagonal; Figure 7 reports the results obtained for a block-diagonal error covariance matrix and a Cholesky decomposition within each block; and Figure 8 reports the generalized FEVDs (GFEVDs) obtained using the regularized estimator of the error covariance matrix. ${ }^{41}$ Each figure reports the 'average' variance decomposition, weighting country-specific decompositions with PPP-GDP weights. We shall now summarize the error variance decompositions that result from these three alternative specifications.

\subsection{Diagonal Covariance Matrix and Orthogonal Decomposition}

The left hand panel of Figure 6 plots the average forecast error variance decomposition of volatility across all countries in our sample under a diagonal error covariance matrix. The

\footnotetext{
${ }^{41}$ The derivation of the FEVDs and GFEVDs is reported in section S3 of the online supplement to the paper.
} 
figure shows that country volatility is driven largely by common financial shocks (blue area with vertical lines) and country-specific volatility shocks (red area with crosses). Combined together, these two shocks explain about 95 percent of the total variance of realized volatility over time. Real common shocks (purple area with diagonal lines) explain less than 5 percent of the total volatility forecast error variance. Country growth shocks, as well as all other 31 country-specific foreign growth shocks in the full model, play essentially no role. These results, therefore, suggest that country volatility is largely driven by global financial shocks and its own country-specific innovations. In other words, according to these results, the component of volatility driven by common or country-specific output shocks, and hence the 'endogenous' component of countryspecific volatility in the sense of Ludvigson et al. (2015) is very small.

\section{Figure 6 Forecast Error Variance Decomposition of Country-specific Shocks - Diagonal Error Covariance Matrix (In Percent)}
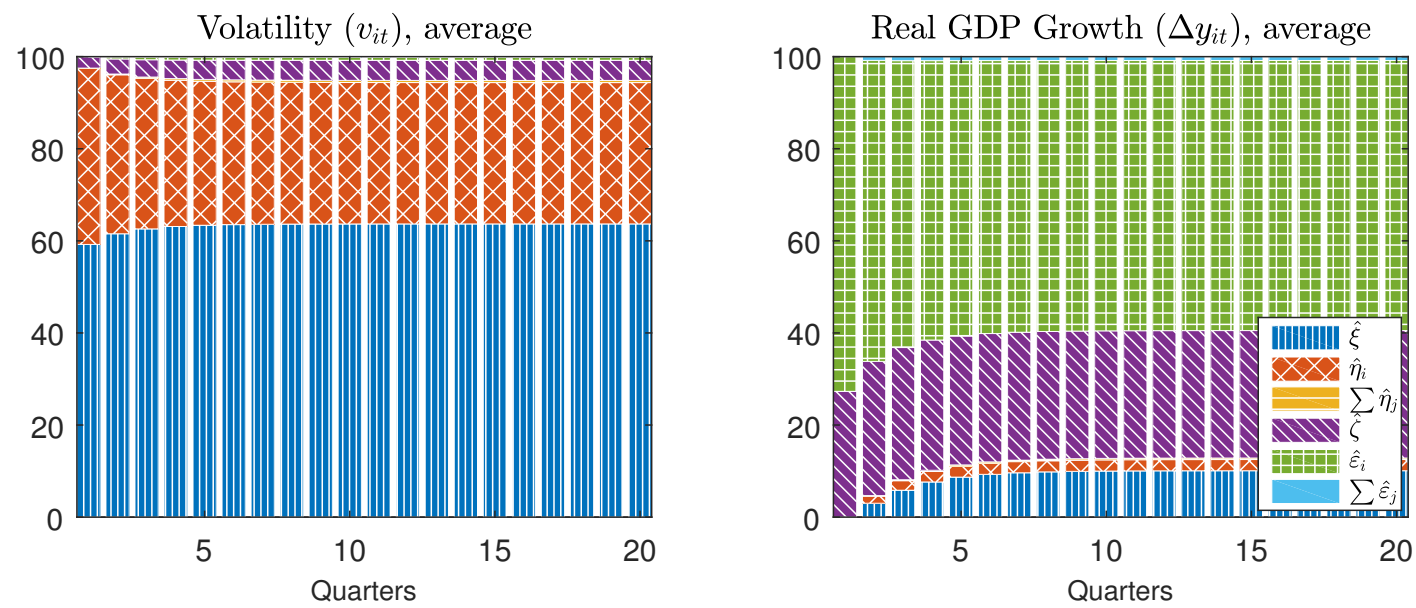

Note. Average across countries with GDP-PPP weights. $\hat{\xi}$ is global financial shock (blue area with vertical lines); $\hat{\eta}_{i}$ is country-specific volatility shock (red area with crosses); $\sum \hat{\eta}_{j}$ is the sum of the contribution of the volatility shocks in the remaining countries (yellow area with horizontal lines); $\hat{\zeta}$ is global real shock (purple area with diagonal lines); $\hat{\varepsilon}_{i}$ is country-specific GDP shock (green areas with squares); $\sum \hat{\varepsilon}_{j}$ is the sum of the contributions of the GDP shocks in the remaining countries (light blue areas with no pattern). Sample period: 1993:Q1-2011:Q2.

It is worth noting that these estimated shares of the forecast error variance of volatility are similar to the central estimates of Ludvigson et al. (2015) for the US. In that study, the share of macroeconomic shocks in the forecast error variance of financial volatility is estimated at just above 5 percent in the long run. However, while Ludvigson et al. (2015) attribute this to the US business cycle (as proxied by a shock to US industrial production), we attribute a similar outcome to the global real shock, which can be interpreted as an international business 
cycle factor, and find that country-specific growth shocks have little or no explanatory power for country-specific volatility. ${ }^{42}$

Consider now the forecast error variance decomposition of GDP growth reported on the right hand side of Figure 6. The figure shows that, on average, the forecast error variance of country specific GDP growth is driven mostly by country-specific growth shocks and global real shocks, with a combined share approaching 90 percent of the total in the long run (green areas with squares and purple area with diagonal lines, respectively). The country-specific growth shock explains more than 60 percent of the total forecast error variance in the long-run, while the real global shock on average explains around 30 percent of the total growth forecast error variance. This is in line with existing results in the international business cycle literature (see, for instance, Kose et al. (2003)). ${ }^{43}$

Global financial shocks explain about 8-10 percent of country-specific growth forecast error variance, on average, in our sample. The importance of these shocks picks up gradually over the forecast horizon and stabilizes within two years. In contrast, the own country-specific volatility shock, explains only a small share of the total forecast error variance of GDP growth, with a weight of about $1-2$ percent, while the combination of all other 31 country-specific volatility shocks in the model explain only a small share of country growth variance. These results clearly illustrate the quantitative importance of distinguishing between common and country-specific volatility shocks.

\subsection{Block-Diagonal Covariance Matrix and Orthogonal Decomposition}

We now maintain the assumption of zero correlations of country-specific shocks (after conditioning on the common shocks) across countries, but allow for a possibly non-zero correlation between volatility and growth within each country. Specifically, we assume that, at the country level, a volatility shock can affect growth within a quarter but not vice versa. This is the assumption typically made in the empirical literature on volatility and the business cycle. So,

\footnotetext{
${ }^{42}$ Results for specific countries, including the United States, are reported in the online supplement. As can be seen from Figures S.6 to S.9 also in the online Supplement, countries behave pretty similarly, with some but limited heterogeneity. The results for the United States, in particular, are similar to those for the average economy reported here.

${ }^{43}$ Notice that these results imply that countries' business cycles remain largely unexplained within our econometric model. Indeed, in the data, there are many shocks at work, and this is captured in our relatively simple empirical framework by the large share of growth forecast error variance accounted for by the own country specific growth shocks.
} 
here, we are 'identifying' exogenous country-specific volatility changes with a Cholesky decomposition of the within-country covariance matrix. We do so by ordering volatility first in the model (62)-(63).

Figure 7 Forecast Error Variance Decomposition of Country-specific Shocks Block Diagonal Error Covariance Matrix (In Percent)
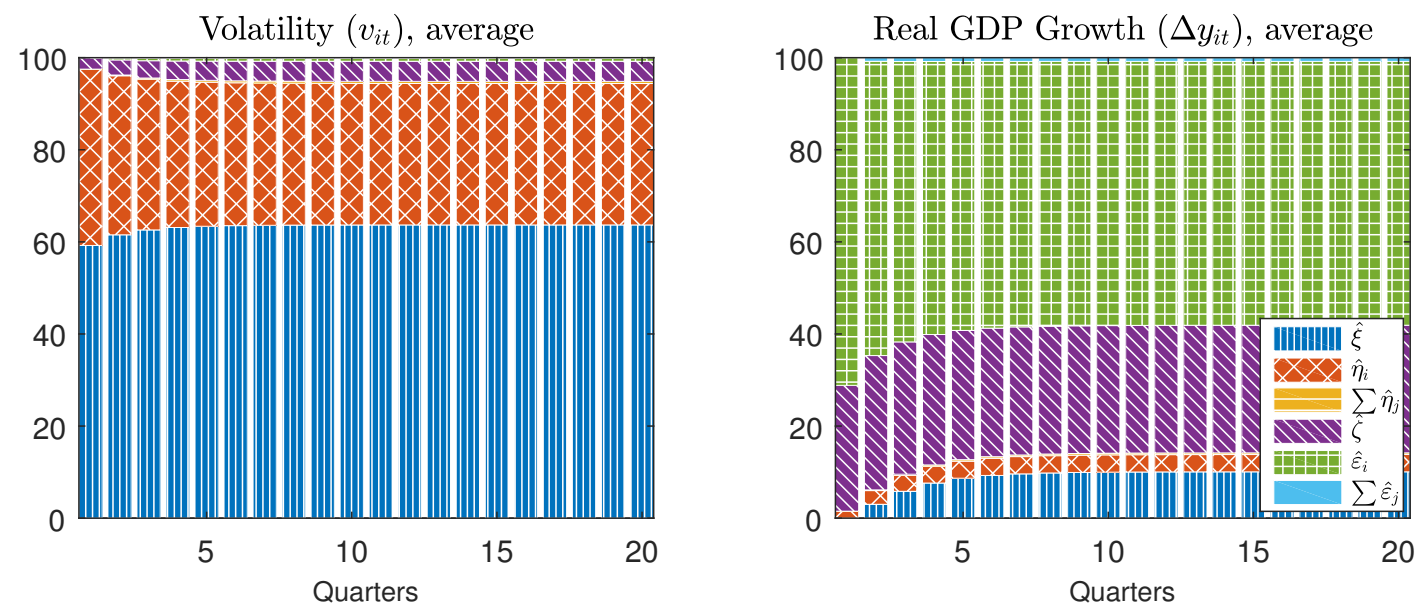

Note. Block-diagonal covariance matrix, with Cholesky decomposition of within-country covariance. Average across countries with GDP-PPP weights. $\hat{\xi}$ is global financial shock (blue area with vertical lines); $\hat{\eta}_{i}$ is countryspecific volatility shock (red area with crosses); $\sum \hat{\eta}_{j}$ is the sum of the contribution of the volatility shocks in the remaining countries (yellow area with horizontal lines); $\hat{\zeta}$ is global real shock (purple area with diagonal lines); $\hat{\varepsilon}_{i}$ is country-specific GDP shock (green areas with squares); $\sum \hat{\varepsilon}_{j}$ is the sum of the contributions of the GDP shocks in the remaining countries (light blue areas with no pattern). Sample period: 1993:Q1-2011:Q2.

The results for this specification are given in Figure 7 and can be seen to be virtually identical to the estimates obtained for the diagonal error covariance matrix reported in Figure 6. This is perhaps not surprising given that the correlations between the country-specific innovations, once the effects of the common shocks are removed, are very small as in Figure 5.

\subsection{Thresholding the Error Covariance Matrix and Generalized Decomposi- tion}

We finally allow for a fully estimated $(64 \times 64)$ correlation matrix, both within and across countries, and compute the GFEVDs. However, given the large size of this matrix, we regularize it by computing a threshold estimator following Bailey et al. (2017), who developed a procedure based on results from the multiple testing literature. Specifically, we first test for the statistical significance of each of the 2016 distinct off-diagonal elements of the $(64 \times 64)$ matrix. We then 
set to zero all those elements that are not statistically significant, using suitably adjusted critical values to allow for the large number of tests that are being carried out. We then finally compute the GVEDs by using the regularized estimates as derived in the online supplement to the paper.

Table 2 below lists all the non-zero correlation pairs. As can be seen, only 50 out of 2016 total off-diagonal elements are statistically different from zero. Of these, about half are positively correlated and the other half are negatively correlated, with an average value that is close to zero. Most notably, there is no surviving within-country contemporaneous correlation between volatility and growth, except for India. There are also very few significant GDP-GDP correlation pairs (i.e., $\hat{\varepsilon}_{i t}$ with $\hat{\varepsilon}_{j t}$ ), with no obvious regional pattern of co-movements. There are a few significant pairs of volatility-volatility correlations (i.e., $\hat{\eta}_{i t}$ with $\hat{\eta}_{j t}$ ), but involving only a handful of countries, with no evidence of a dominant role for the United States. Finally, there are a few significant GDP-volatility correlation pairs (i.e., $\hat{\varepsilon}_{j t}$ with $\hat{\eta}_{i t}$ ) for a few countries, like Belgium, China, France, Italy and the Netherlands, again revealing no specific patterns.

The estimated generalized forecast error variance decompositions (GFEVDs), reported in Figure 8, are consistent with those obtained assuming a diagonal or block-diagonal error covariance matrix. ${ }^{44}$ Relative to the results with diagonal or block-diagonal covariance matrix in Figures 6 and 7, the contribution of foreign country-specific volatility (growth) shocks, $\sum \hat{\eta}_{j}$ $\left(\sum \hat{\varepsilon}_{j}\right)$, to domestic volatility (growth) is now larger, but the spillover effects of foreign volatility shocks to growth (and foreign growth shocks to volatility) remain negligible. Moreover, global financial shocks and domestic country-specific volatility shocks continue to explain the bulk of the forecast error variance of volatility. Similarly, real global shocks and the country-specific growth shocks remain the main drivers of the forecast error variance of growth.

\footnotetext{
${ }^{44}$ Notice here that the GFEVDs need not to sum to 100 as the underlying shocks are not orthogonal.
} 
Table 2 Non-Zero Elements of the Regularized ERror Covariance Matrix Estimate

\begin{tabular}{|c|c|c|c|c|c|c|}
\hline \multicolumn{3}{|c|}{ All Significant } & \multicolumn{3}{|c|}{ Between-county } & \multirow{2}{*}{$\begin{array}{c}\text { Within-country } \\
\hat{\varepsilon}_{i t}, \hat{\eta}_{i t} \\
\end{array}$} \\
\hline Country - I & ariable Pairs & Corr & $\hat{\varepsilon}_{i t}, \hat{\varepsilon}_{j t}$ & $\hat{\eta}_{i t}, \hat{\eta}_{j t}$ & $\hat{\varepsilon}_{i t}, \hat{\eta}_{j t}$ & \\
\hline $\operatorname{ARG}\left(\hat{\eta}_{i t}\right)$ & $\mathrm{ZAF}\left(\hat{\eta}_{j t}\right)$ & 0.46 & & & & \\
\hline $\operatorname{AUT}\left(\hat{\varepsilon}_{i t}\right)$ & PHL $\left(\hat{\varepsilon}_{j t}\right)$ & -0.43 & AUT, PHL & & & \\
\hline $\operatorname{BEL}\left(\hat{\eta}_{i t}\right)$ & $\operatorname{ITA}\left(\hat{\eta}_{j t}\right)$ & 0.51 & & BEL, ITA & & \\
\hline $\operatorname{BEL}\left(\hat{\eta}_{i t}\right)$ & $\operatorname{NLD}\left(\hat{\eta}_{j t}\right)$ & 0.60 & & BEL, NLD & & \\
\hline $\operatorname{BEL}\left(\hat{\eta}_{i t}\right)$ & $\operatorname{CHE}\left(\hat{\eta}_{j t}\right)$ & 0.51 & & BEL, CHE & & \\
\hline $\operatorname{BEL}\left(\hat{\eta}_{i t}\right)$ & $\operatorname{GBR}\left(\hat{\eta}_{j t}\right)$ & 0.54 & & BEL, GBR & & \\
\hline $\operatorname{BEL}\left(\hat{\varepsilon}_{i t}\right)$ & $\mathrm{CHN}\left(\hat{\varepsilon}_{j t}\right)$ & -0.40 & BEL, CHN & & & \\
\hline $\operatorname{BRA}\left(\hat{\eta}_{i t}\right)$ & $\operatorname{MEX}\left(\hat{\eta}_{j t}\right)$ & 0.56 & & BRA, MEX & & \\
\hline $\operatorname{BRA}\left(\hat{\varepsilon}_{i t}\right)$ & $\operatorname{CHN}\left(\hat{\varepsilon}_{j t}\right)$ & -0.44 & BRA, CHN & & & \\
\hline CAN $\left(\hat{\eta}_{i t}\right)$ & $\operatorname{NOR}\left(\hat{\eta}_{j t}\right)$ & 0.40 & & CAN, NOR & & \\
\hline CHN $\left(\hat{\eta}_{i t}\right)$ & $\operatorname{FRA}\left(\hat{\eta}_{j t}\right)$ & -0.58 & & CHN, FRA & & \\
\hline $\mathrm{CHN}\left(\hat{\eta}_{i t}\right)$ & $\operatorname{ITA}\left(\hat{\eta}_{j t}\right)$ & -0.42 & & CHN, ITA & & \\
\hline $\mathrm{CHN}\left(\hat{\eta}_{i t}\right)$ & $\operatorname{NLD}\left(\hat{\eta}_{j t}\right)$ & -0.46 & & CHN, NLD & & \\
\hline $\operatorname{CHN}\left(\hat{\eta}_{i t}\right)$ & $\operatorname{ESP}\left(\hat{\eta}_{i t}\right)$ & -0.41 & & CHN, ESP & & \\
\hline $\mathrm{CHN}\left(\hat{\eta}_{i t}\right)$ & $\operatorname{SWE}\left(\hat{\eta}_{j t}\right)$ & -0.40 & & CHN, SWE & & \\
\hline CHN $\left(\hat{\eta}_{i t}\right)$ & $\operatorname{CHE}\left(\hat{\eta}_{j t}\right)$ & -0.45 & & CHN, CHE & & \\
\hline $\mathrm{CHN}\left(\hat{\eta}_{i t}\right)$ & $\operatorname{GBR}\left(\hat{\eta}_{j t}\right)$ & -0.49 & & CHN, GBR & & \\
\hline CHN $\left(\hat{\eta}_{i t}\right)$ & $\operatorname{USA}\left(\hat{\eta}_{j t}\right)$ & -0.57 & & CHN, USA & & \\
\hline $\operatorname{CHN}\left(\hat{\varepsilon}_{i t}\right)$ & FRA $\left(\hat{\varepsilon}_{j t}\right)$ & -0.39 & CHN, FRA & & & \\
\hline $\mathrm{CHN}\left(\hat{\varepsilon}_{i t}\right)$ & JPN $\left(\hat{\eta}_{j t}\right)$ & 0.55 & & & CHN, JPN & \\
\hline $\operatorname{CHN}\left(\hat{\varepsilon}_{i t}\right)$ & USA $\left(\hat{\varepsilon}_{j t}\right)$ & -0.51 & CHN, USA & & & \\
\hline $\operatorname{FIN}\left(\hat{\eta}_{i t}\right)$ & $\operatorname{KOR}\left(\hat{\varepsilon}_{j t}\right)$ & -0.41 & & & FIN, KOR & \\
\hline FIN $\left(\hat{\eta}_{i t}\right)$ & $\operatorname{TUR}\left(\hat{\varepsilon}_{j t}\right)$ & 0.41 & & & FIN, TUR & \\
\hline $\operatorname{FRA}\left(\hat{\eta}_{i t}\right)$ & $\operatorname{DEU}\left(\hat{\eta}_{j t}\right)$ & 0.50 & & FRA, DEU & & \\
\hline FRA $\left(\hat{\eta}_{i t}\right)$ & $\operatorname{IND}\left(\hat{\eta}_{j t}\right)$ & -0.46 & & FRA, IND & & \\
\hline FRA $\left(\hat{\eta}_{i t}\right)$ & IDN $\left(\hat{\eta}_{i t}\right)$ & -0.39 & & FRA, IDN & & \\
\hline FRA $\left(\hat{\eta}_{i t}\right)$ & $\operatorname{ITA}\left(\hat{\eta}_{j t}\right)$ & 0.46 & & FRA, ITA & & \\
\hline FRA $\left(\hat{\eta}_{i t}\right)$ & $\operatorname{NLD}\left(\hat{\eta}_{j t}\right)$ & 0.63 & & FRA, NLD & & \\
\hline FRA $\left(\hat{\eta}_{i t}\right)$ & $\operatorname{ESP}\left(\hat{\eta}_{j t}\right)$ & 0.61 & & FRA, ESP & & \\
\hline FRA $\left(\hat{\eta}_{i t}\right)$ & $\operatorname{SWE}\left(\hat{\eta}_{j t}\right)$ & 0.51 & & FRA, SWE & & \\
\hline FRA $\left(\hat{\eta}_{i t}\right)$ & $\operatorname{CHE}\left(\hat{\eta}_{i t}\right)$ & 0.55 & & FRA, CHE & & \\
\hline FRA $\left(\hat{\eta}_{i t}\right)$ & $\operatorname{GBR}\left(\hat{\eta}_{j t}\right)$ & 0.71 & & FRA, GBR & & \\
\hline $\operatorname{IND}\left(\hat{\eta}_{i t}\right)$ & $\operatorname{NLD}\left(\hat{\eta}_{i t}\right)$ & -0.39 & & IND, NLD & & \\
\hline $\operatorname{IND}\left(\hat{\eta}_{i t}\right)$ & $\operatorname{GBR}\left(\hat{\eta}_{j t}\right)$ & -0.49 & & IND, GBR & & \\
\hline $\operatorname{IND}\left(\hat{\eta}_{i t}\right)$ & USA $\left(\hat{\eta}_{i t}\right)$ & -0.46 & & IND, USA & & \\
\hline $\operatorname{IDN}\left(\hat{\eta}_{i t}\right)$ & IDN $\left(\hat{\varepsilon}_{j t}\right)$ & -0.43 & & & & IDN, IDN \\
\hline $\operatorname{ITA}\left(\hat{\eta}_{i t}\right)$ & $\operatorname{NLD}\left(\hat{\eta}_{j t}\right)$ & 0.60 & & ITA, NLD & & \\
\hline ITA $\left(\hat{\eta}_{i t}\right)$ & $\operatorname{ESP}\left(\hat{\eta}_{j t}\right)$ & 0.61 & & ITA, ESP & & \\
\hline ITA $\left(\hat{\eta}_{i t}\right)$ & $\operatorname{GBR}\left(\hat{\eta}_{j t}\right)$ & 0.46 & & ITA, GBR & & \\
\hline $\operatorname{KOR}\left(\hat{\varepsilon}_{i t}\right)$ & $\operatorname{MYS}\left(\hat{\varepsilon}_{j t}\right)$ & 0.58 & KOR, MYS & & & \\
\hline $\operatorname{KOR}\left(\hat{\varepsilon}_{i t}\right)$ & THA $\left(\hat{\varepsilon}_{j t}\right)$ & 0.47 & KOR, THA & & & \\
\hline $\operatorname{MYS}\left(\hat{\eta}_{i t}\right)$ & $\operatorname{SWE}\left(\hat{\eta}_{j t}\right)$ & -0.39 & & MYS, SWE & & \\
\hline $\operatorname{MYS}\left(\hat{\varepsilon}_{i t}\right)$ & $\operatorname{NOR}\left(\hat{\eta}_{j t}\right)$ & -0.41 & & & MYS, NOR & \\
\hline $\operatorname{NLD}\left(\hat{\eta}_{i t}\right)$ & $\operatorname{ESP}\left(\hat{\eta}_{j t}\right)$ & 0.50 & & NLD, ESP & & \\
\hline $\operatorname{NLD}\left(\hat{\eta}_{i t}\right)$ & $\operatorname{CHE}\left(\hat{\eta}_{j t}\right)$ & 0.70 & & NLD, CHE & & \\
\hline $\operatorname{NLD}\left(\hat{\eta}_{i t}\right)$ & $\operatorname{GBR}\left(\hat{\eta}_{j t}\right)$ & 0.74 & & NLD, GBR & & \\
\hline $\operatorname{NOR}\left(\hat{\varepsilon}_{i t}\right)$ & $\operatorname{THA}\left(\hat{\eta}_{j t}\right)$ & 0.40 & & & NOR, THA & \\
\hline PHL $\left(\hat{\eta}_{i t}\right)$ & $\operatorname{SGP}\left(\hat{\eta}_{j t}\right)$ & 0.44 & & PHL, SGP & & \\
\hline $\operatorname{SGP}\left(\hat{\eta}_{i t}\right)$ & $\mathrm{USA}\left(\hat{\eta}_{j t}\right)$ & -0.42 & & SGP, USA & & \\
\hline $\operatorname{CHE}\left(\hat{\eta}_{i t}\right)$ & $\operatorname{GBR}\left(\hat{\eta}_{j t}\right)$ & 0.66 & & CHE, GBR & & \\
\hline
\end{tabular}

Note. Non-zero elements of the regularized error covariance matrix estimate proposed by Bailey et al. (2017). Sample period: 1993:Q1-2011:Q2. 
We interpret the above results as strong evidence of robustness of our conclusions reached by assuming a diagonal or block-diagonal covariance matrix. In particular, it remains the case that common or country-specific output growth shocks have no important role for volatility, and home and foreign country-specific volatility shocks have little quantitative consequence for output growth.

Figure 8 Generalized Forecast Error Variance Decomposition of Country-Specific Shocks - Estimation of Regularized Full Error Covariance MAtrix (In PERCENT)
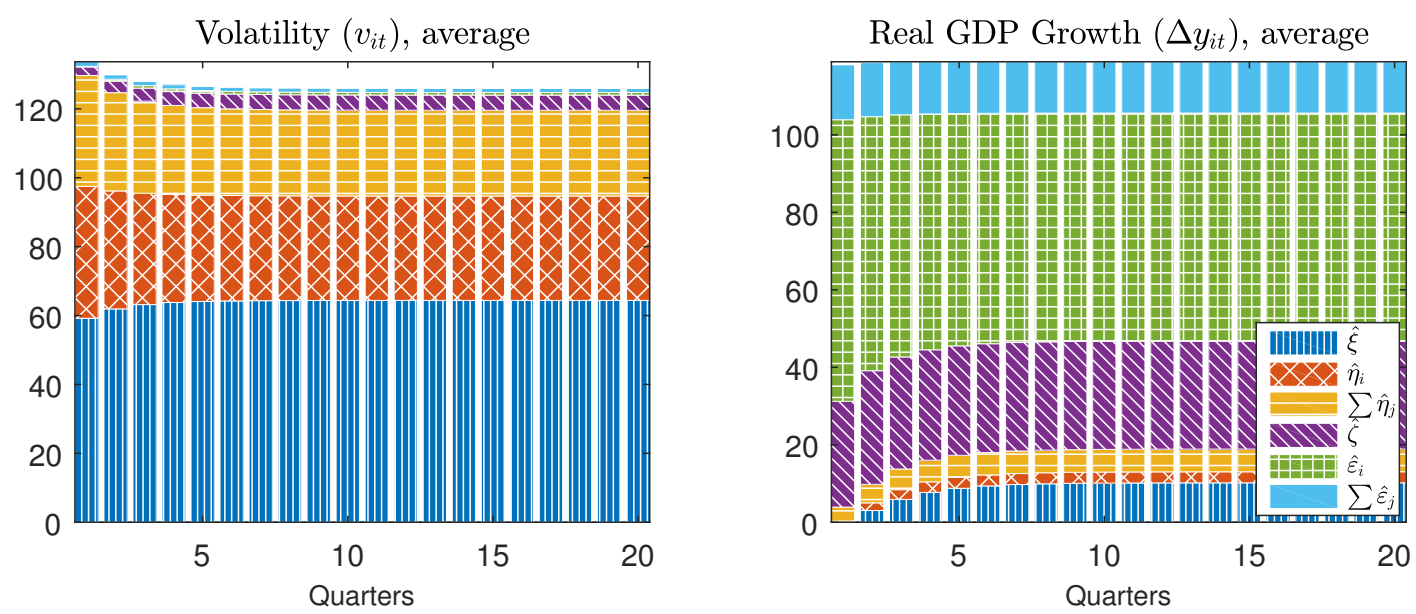

Note. Threshold estimator of the population covariance matrix. Average across countries with GDP-PPP weights. $\hat{\xi}$ is global financial shock (blue area with vertical lines); $\hat{\eta}_{i}$ is country-specific volatility shock (red area with crosses); $\sum \hat{\eta}_{j}$ is the sum of the contribution of the volatility shocks in the remaining countries (yellow area with horizontal lines); $\hat{\zeta}$ is global real shock (purple area with diagonal lines); $\hat{\varepsilon}_{i}$ is country-specific GDP shock (green areas with squares); $\sum \hat{\varepsilon}_{j}$ is the sum of the contributions of the GDP shocks in the remaining countries (light blue areas with no pattern). Sample period: 1993:Q1-2011:Q2.

\section{The Transmission of Global Real and Financial Shocks}

The last step of our empirical analysis is the computation of impulse responses of countryspecific volatility and growth to global real and financial shocks, $\hat{\zeta}_{t}$ and $\hat{\xi}_{t}$. While forecast error variance decompositions speak to the importance of a particular shock for the time-variation of the endogenous variables relative to other shocks in the model, impulse responses provide information on the size of the effects of the shocks and their transmission across variables and countries.

Figure 9 displays a weighted average of the country-specific impulse responses using PPP- 
GDP weights (solid line), together with two-standard deviation error bands (shaded areas). The error bands are computed based on the dispersion of the impulse responses across countries. ${ }^{45}$ We focus on the effects of positive unit (one-standard deviation) real and financial shocks, $\hat{\zeta}_{t}$ and $\hat{\xi}_{t} \cdot{ }^{46}$

\section{Figure 9 Average Country Volatility and Growth Responses to Real and Financial Factor Shocks (In Percent)}
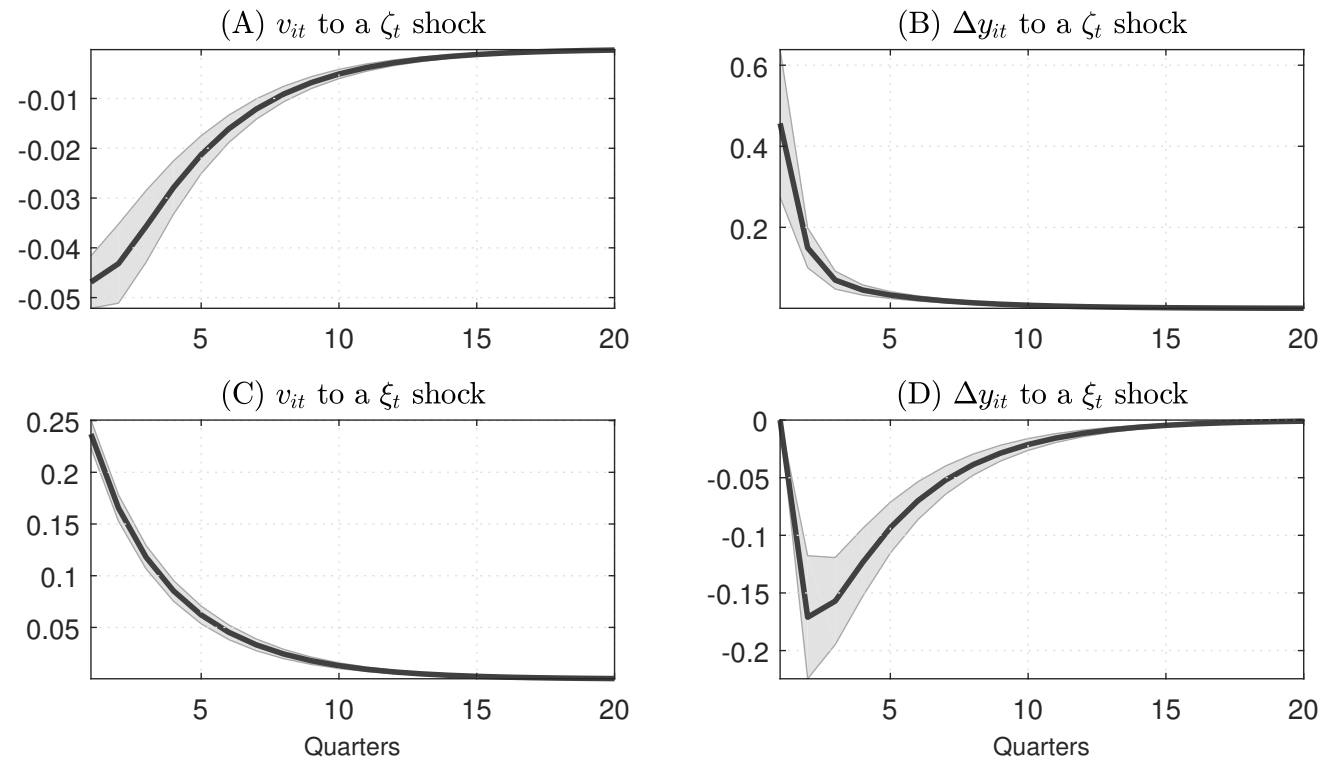

Note. Average impulse responses to one-standard deviation real and financial shocks, $\hat{\zeta}_{t}$ and $\hat{\xi}_{t}$. The solid lines are the PPP-GDP weighted averages of the country-specific responses. The shaded areas are the two standard deviations confidence intervals. See equations (S3.12) and (S3.13) for the derivations and Figure S.12 for the country-specific responses. Sample period: 1993:Q1-2011:Q2.

Panels (A) and (B) of Figure 9 display the average across countries of the volatility and growth responses to a real global shock. These figures show that a positive real global shock increases output growth and lowers volatility. This reflects an endogenous volatility response to the fundamental improvements in the world economy. Note that the error bands around the average responses are very tight, reflecting relatively homogeneous country responses. In fact, as can be seen from Figure S.12, provided in the online supplement, the impulse responses have a similar shape for most countries. The average impact of this shock on country volatilities is one order of magnitude smaller than its impact on country output growth, but it is quite persistent,

\footnotetext{
${ }^{45}$ The derivation of the average impulse response functions to common factor shocks and their error bands is provided in the online supplement S3, equations (S3.12) and (S3.13). The estimated country-specific responses to the two common shocks are reported in Figure S.12 in the online Supplement to the paper.

${ }^{46}$ Recall that these shocks are orthogonal to all other shocks in the model and to each other by construction.
} 
taking more than three years for the effects of the shocks to vanish completely. Panel (B) also shows that, on average, country growth loads positively on $\hat{\zeta}_{t}$, with persistent effects up to 8-10 quarters, as one would expect. Country output growth increase by about half a percentage point following a one-standard error change in $\hat{\zeta}_{t}$. This is consistent with the existing evidence on the international business cycle, which attributes an important role to a world factor, along with regional and country-specific factors, in driving the business cycle (e.g., Kose et al. (2003)).

Panels (C) and (D) of Figure 9 report the responses of volatility and growth to a positive global financial shock, $\hat{\xi}_{t}$. These average responses suggest that a positive shock to $\hat{\xi}_{t}$ is 'bad news' for the world economy, as volatility increases and growth declines. For a one-standard deviation shock to the common financial factor, volatility increases by 25 basis points, while growth declines by about 15 basis points within two quarters after the shock. ${ }^{47}$ Although smaller than the growth response to the real common factor shock in panel B, the average growth response to the global financial shock in panel D is of the same order of magnitude, and hence quantitatively sizable. The average responses to the common financial shock are also very persistent, but there is much more heterogeneity in the country growth responses, as can be seen from Figure S.12 provided in the online supplement. Therefore, these impulse responses suggest that, even though common financial shocks may not explain a very large share of the forecast error variance of output growth over time, they can cause large and persistent global recessions.

Impulse responses to country-specific shocks have qualitatively similar pattern of transmission but, as one would expect given the forecast error variance results, are quantitatively much smaller than responses to common shocks. ${ }^{48}$

The pattern of shock transmissions in Figure 9 is consistent with country volatility increasing in response to the large declines in the world output in the second part of 2008, and the world recession being amplified by the exceptionally large common financial shock in the fourth quarter of 2008, and the first quarter of 2009. The transmission in Figure 9 can also help in explaining the seemingly puzzling coexistence of high policy volatility (as in Baker et al. (2016)) and low equity market volatility after the beginning of the Trump administration with a combination of a real and a financial shock partially offsetting each other.

\footnotetext{
${ }^{47}$ Note that the delayed growth response to the global financial shock follows from our identification assumptions, but it is not imposed directly on country-specific models.

${ }^{48}$ These are not reported but are available from the authors on request.
} 


\section{Conclusions}

Empirical measures of uncertainty behave countercyclically in most countries of the world, but economic theory suggests that causation can run both ways. In this paper, we study the interrelation between realized equity price volatility and GDP growth without imposing a priori restrictions on the direction of economic causation. We develop a multi-country version of the Lucas tree model with time-varying volatility and derive expressions for country-specific output growth, equity returns and their realized volatilities in terms of innovations to a global technology or growth factor and its higher-order moments. We show that both a level (or first-order) common component and higher-order common components are required to explain the cross country variations of realized volatility, but only the level common component is required for explaining cross-country differences in output growth. Using this theoretical insight, we estimate a multi-country econometric model in output growth and realized volatilities for 32 countries over the period 1993Q1-2011Q2. Common real and financial shocks are identified assuming that volatility and growth are driven by two common factors. By taking a multi-country approach, as opposed to studying a single economy in isolation, we can identify and estimate these two factors. Our identification strategy exploits different patterns in the correlations of volatility and output growth innovations across countries. Evidence based on the cross-country correlations of the raw data and estimated innovations accords with the assumptions made to achieve identification.

Empirically, we report three main sets of findings. First, shocks to the real common factor account for most of the unconditional correlation between volatility and growth in all but few emerging market economies. Second, the share of forecast error variance of volatility explained by the real common factor shock and by country-specific growth shocks is less than 5 percent. Third, shocks to the financial common factor explain about 10 percent of the growth forecast error variance, while country-specific volatility shocks explain only about 1-2 percent of countryspecific growth forecast error variance. Moreover, when a shock to the financial common factor is realized, its negative impact on country-specific growth is large and persistent as typically estimated in the existing literature.

These results imply that the endogenous component of country volatility is small and that uncertainty is more important for the business cycle when the shocks are global in nature. 


\section{References}

ABel, A. B. (1983): "Optimal Investment under Uncertainty," American Economic Review, $73,228-33$.

AiYAGari, S. R. (1993): "Explaining financial market facts: the importance of incomplete markets and transaction costs," Quarterly Review, 17-31.

Andersen, T. G., T. Bollerslev, F. X. Diebold, and P. Labys (2001): "The Distribution of Realized Exchange Rate Volatility," Journal of the American Statistical Association, 96, $42-55$.

(2003): “Modeling and Forecasting Realized Volatility," Econometrica, 71, 579-625.

Arellano, C., Y. Bai, And P. Kehoe (2012): "Financial Markets and Fluctuations in Uncertainty," Unpublished manuscript.

Bachmann, R. And C. BAyer (2013): “'Wait-and-See' business cycles?” Journal of Monetary Economics, 60, 704-719.

Bachmann, R., S. Elstner, And E. R. Sims (2013): "Uncertainty and Economic Activity: Evidence from Business Survey Data," American Economic Journal: Macroeconomics, 5, 217-249.

Backus, D. K., P. J. Kehoe, and F. E. Kydland (1992): "International Real Business Cycles," Journal of Political Economy, 100, 745-775.

BAI, J. AND S. NG (2002): "Determining the Number of Factors in Approximate Factor Models," Econometrica, 70, 191-221.

Bailey, N., G. Kapetanios, and M. H. Pesaran (2016): "Exponent of Cross-Sectional Dependence: Estimation and Inference," Journal of Applied Econometrics, 31, 929-960.

Bailey, N., M. H. Pesaran, And L. V. Smith (2017): "A Multiple Testing Approach to the Regularisation of Large Sample Correlation Matrices," Unpublished.

Baker, S. R. And N. Bloom (2013): "Does Uncertainty Reduce Growth? Using Disasters as Natural Experiments," NBER Working Papers 19475, National Bureau of Economic Research, Inc.

Baker, S. R., N. Bloom, and S. J. Davis (2016): "Measuring Economic Policy Uncertainty," The Quarterly Journal of Economics, 131, 1593-1636.

Bansal, R., V. Khatchatrian, and A. Yaron (2005): "Interpretable asset markets?" European Economic Review, 49, 531-560.

Barndorff-Nielsen, O. E. ANd N. Shephard (2002): "Estimating quadratic variation using realized variance," Journal of Applied Econometrics, 17, 457-477.

- (2004): "Econometric Analysis of Realized Covariation: High Frequency Based Covariance, Regression, and Correlation in Financial Economics," Econometrica, 72, 885-925.

BAsu, S. AND B. Bundick (2017): "Uncertainty Shocks in a Model of Effective Demand," Econometrica, 85, 937-958. 
Baxter, M. And M. J. Crucini (1995): "Business Cycles and the Asset Structure of Foreign Trade," International Economic Review, 36, 821-854.

Berger, D., I. Dew-Becker, And S. Giglio (2017): "Uncertainty Shocks as Second-Moment News Shocks," NBER Working Papers 23796, National Bureau of Economic Research, Inc.

Bernanke, B. S. (1983): "Irreversibility, Uncertainty, and Cyclical Investment," The Quarterly Journal of Economics, 98, 85-106.

Black, F. (1976): "Studies of stock price volatility changes," Proceedings of the 1976 Meetings of the Business and Economics Statistics Section, American Statistical Association, 177-181.

Bloom, N. (2009): "The Impact of Uncertainty Shocks," Econometrica, 77, 623-685.

(2014): "Fluctuations in Uncertainty," Journal of Economic Perspectives, 28, 153-176.

Bloom, N., S. Bond, And J. V. Reenen (2007): "Uncertainty and Investment Dynamics," Review of Economic Studies, 74, 391-415.

Bloom, N., M. Floetotto, N. Jaimovich, I. Saporta-Eksten, and S. J. Terry (2012): "Really Uncertain Business Cycles," NBER Working Papers 18245, National Bureau of Economic Research, Inc.

Born, B. AND J. Pfeifer (2014): "Policy risk and the business cycle," Journal of Monetary Economics, 68, 68-85.

Campbell, J. Y., M. Lettau, B. G. Malkiel, and Y. Xu (2001): "Have Individual Stocks Become More Volatile? An Empirical Exploration of Idiosyncratic Risk," Journal of Finance, $56,1-43$.

Campbell, J. Y. And R. J. Shiller (1988): "The Dividend-Price Ratio and Expectations of Future Dividends and Discount Factors," Review of Financial Studies, 1, 195-228.

Carriere-Swallow, Y. and L. F. Cespedes (2013): "The impact of uncertainty shocks in emerging economies," Forthcoming Journal of international Economics.

Carriero, A., T. Clark, and M. Marcellino (2017): "Assessing International Commonality in Macroeconomic Uncertainty and its Effects," Unpublished.

Chamberlain, G. And M. Rothschild (1982): "Arbitrage, Factor Structure, and MeanVariance Analysis on Large Asset Markets," Tech. rep.

Christiano, L., R. Motto, and M. Rostagno (2014): "Risk Shocks," American Economic Review, 104, 27-65.

Chudik, A. And M. H. Pesaran (2013): "Econometric Analysis of High Dimensional VARs Featuring a Dominant Unit," Econometric Reviews, 32, 592-649.

(2015): "Common correlated effects estimation of heterogeneous dynamic panel data models with weakly exogenous regressors," Journal of Econometrics, 188, 393-420.

Chudik, A., M. H. Pesaran, And E. Tosetti (2011): "Weak and strong cross-section dependence and estimation of large panels," Econometrics Journal, 14, C45-C90. 
Clark, T. E., A. Carriero, and M. Massimiliano (2016): "Measuring Uncertainty and Its Impact on the Economy," Working Paper 1622, Federal Reserve Bank of Cleveland.

Colacito, R. And M. M. Croce (2011): "Risks for the Long Run and the Real Exchange Rate," Journal of Political Economy, 119, 153-181.

Diebold, F. And K. Yilmaz (2010): "Macroeconomic Volatility and Stock Market Volatility, Worldwide," in Volatility and Time Series Econometrics: Essays in Honor of Robert F. Engle, ed. by J. R. T. Bollerslev and M. Watson, Oxford: Oxford University Press, 97-116.

Dixit, A. K. AND R. S. Pindyck (1994): Investment under Uncertainty, Princeton University Press.

Fernandez-Villaverde, J., P. Guerron-Quintana, J. F. Rubio-Ramirez, and M. Uribe (2011): "Risk Matters: The Real Effects of Volatility Shocks," American Economic Review, 101, 2530-61.

Fostel, A. And J. Geanakoplos (2012): "Why Does Bad News Increase Volatility and Decrease Leverage?" Journal of Economic Theory, 147, 501-525.

French, K. R., G. W. Schwert, and R. F. Stambaugh (1987): "Expected stock returns and volatility," Journal of Financial Economics, 19, 3-29.

Gilchrist, S., J. Sim, And E. Zakrajsek (2013): "Uncertainty, Financial Frictions, and Irreversible Investment," Unpublished manuscript.

Gilchrist, S. And E. Zakrajsek (2012): "Credit Spreads and Business Cycle Fluctuations," American Economic Review, 102, 1692-1720.

Gorodnichenko, Y. And S. NG (2017): "Level and Volatility Factors in Macroeconomic Data," NBER Working Papers 23672, National Bureau of Economic Research, Inc.

Hartman, R. (1976): "Factor Demand with Output Price Uncertainty," American Economic Review, 66, 675-81.

Herskovic, B., B. Kelly, H. Lustig, And S. Van Nieuwerburgh (2016): "The common factor in idiosyncratic volatility: Quantitative asset pricing implications," Journal of Financial Economics, 119, 249-283.

Hirata, H., M. A. Kose, C. Otrok, and M. E. Terrones (2012): "Global House Price Fluctuations: Synchronization and Determinants," in NBER International Seminar on Macroeconomics 2012, National Bureau of Economic Research, Inc, NBER Chapters.

Ilut, C., M. Kehrig, And M. Schneider (2017): "Slow to Hire, Quick to Fire: Employment Dynamics with Asymmetric Responses to News," Forthcoming in the Journal of Political Economy.

Jurado, K., S. C. Ludvigson, And S. NG (2015): "Measuring Uncertainty," American Economic Review, 105, 1177-1216.

Kimball, M. S. (1990): "Precautionary Saving in the Small and in the Large," Econometrica, $58,53-73$. 
Kose, M. A., C. Otrok, and C. H. Whiteman (2003): "International Business Cycles: World, Region, and Country-Specific Factors," American Economic Review, 93, 1216-1239.

Leahy, J. V. And T. M. Whited (1996): "The Effect of Uncertainty on Investment: Some Stylized Facts," Journal of Money, Credit and Banking, 28, 64-83.

Lee, K., M. H. Pesaran, And R. Smith (1997): "Growth and Convergence in Multi-country Empirical Stochastic Solow Model," Journal of Applied Econometrics, 12, 357-392.

LEWIS, K. K. AND E. X. LiU (2015): "Evaluating international consumption risk sharing gains: An asset return view," Journal of Monetary Economics, 71, 84-98.

Lucas, Robert E, J. (1978): "Asset Prices in an Exchange Economy," Econometrica, 46, 1429-1445.

Ludvigson, S. C., S. MA, And S. NG (2015): "Uncertainty and Business Cycles: Exogenous Impulse or Endogenous Response?" NBER Working Papers 21803, National Bureau of Economic Research, Inc.

Mirman, L. J. (1971): "Uncertainty and Optimal Consumption Decisions," Econometrica, 39, $179-85$.

Nakamura, E., D. Sergeyev, And J. Steinsson (2017): "Growth-Rate and Uncertainty Shocks in Consumption: Cross-Country Evidence," American Economic Journal: Macroeconomics, 9, 1-39.

Obstfeld, M. And K. S. Rogoff (1996): Foundations of International Macroeconomics, vol. 1 of MIT Press Books, The MIT Press.

OI, W. (1961): "The desirability of price stability under perfect competition," Econometrica, $29(1), 58-64$.

Pesaran, H. H. And Y. Shin (1998): "Generalized impulse response analysis in linear multivariate models," Economics Letters, 58, 17-29.

Pesaran, M. H. (2015): Time Series and Panel Data Econometrics, no. 9780198759980 in OUP Catalogue, Oxford University Press.

Pesaran, M. H. And A. Chudik (2014): "Aggregation in large dynamic panels," Journal of Econometrics, 178, 273-285.

Popescu, A. And F. R. Smets (2010): "Uncertainty, Risk-taking, and the Business Cycle in Germany," CESifo Economic Studies, 56, 596-626.

Ramey, G. And V. A. Ramey (1995): "Cross-Country Evidence on the Link between Volatility and Growth," American Economic Review, 85, 1138-51.

Renault, E., T. van der Heijden, And B. J. Werker (2016): "Arbitrage Pricing Theory for Idiosyncratic Variance Factors," Unpublished.

REY, H. (2013): "Dilemma not trilemma: the global cycle and monetary policy independence," Proceedings - Economic Policy Symposium - Jackson Hole, -, 1-2. 
Ross, S. A. (1976): "The arbitrage theory of capital asset pricing," Journal of Economic Theory, 13, 341-360.

Rossi, B. And T. Sekhposyan (2015): "Macroeconomic Uncertainty Indices Based on Nowcast and Forecast Error Distributions," American Economic Review, 105, 650-655.

Schwert, G. W. (1989a): "Business cycles, financial crises, and stock volatility," CarnegieRochester Conference Series on Public Policy, 31, 83-125.

(1989b): "Why Does Stock Market Volatility Change over Time?" Journal of Finance, $44,1115-1153$.

TESAR, L. L. (1995): "Evaluating the gains from international risk sharing," Carnegie-Rochester Conference Series on Public Policy, 42, 95-143.

Van Nieuwerburgh, S. And L. Veldkamp (2006): "Learning asymmetries in real business cycles," Journal of Monetary Economics, 53, 753-772.

Zarnowitz, V. And L. A. Lambros (1987): "Consensus and Uncertainty in Economic Prediction," Journal of Political Economy, 95, 591-621. 


\section{Appendix: Derivations and Data Sources}

\section{A Mathematical Derivations}

\section{A.1 Country-specific Growth Process}

One way to motivate the output growth specification, (1), is as follows. Assume a standard CobbDouglas production function in terms of output per worker and denote $\left(Y_{i t} / L_{i t}\right)=\exp \left(\tilde{y}_{i t}\right)$, real GDP per capita, $A_{i t}=\exp \left(a_{i t}\right)$ the country-specific level of technology, $L_{i t}$ the labor force, and $K_{i t}$ the capital stock in country $i$ so that we have:

$$
\tilde{y}_{i t}=\ln \left(Y_{i t} / L_{i t}\right)=a_{i t}+\alpha_{i} \ln \left(K_{i t} / L_{i t}\right)=a_{i t}+\alpha_{i} \log \left(k_{i t}\right)
$$

for $i=1,2, \ldots, N$. Further assume that the processes for $L_{i t}$ and $a_{i t}$ are exogenously given by

$$
\ln \left(L_{i t}\right)-\ln \left(L_{i, t-1}\right)=n_{i} \text {, and } a_{i t}=a_{i 0}+\tilde{g}_{i} t+\gamma_{i} a_{t}+e_{i t}
$$

where growth of labour force, $n_{i}$, is assumed to be fixed, $a_{i 0}$ is an initial condition, $\tilde{g}_{i}$ is a deterministic growth component of $a_{i t}, a_{t}$ is the log-level of a stochastic common technology factor, and $e_{i t}$ is the country-specific technology shock, with $\gamma_{i}$ measuring the extent to which country $i$ is exposed to the global technology factor $a_{t}$. A key result from the stochastic growth literature is that, for all $i, \log \left(k_{i t}\right)$ is ergodic and stationary, in the sense that as $t$ tends to infinity, $\log \left(k_{i t}\right)$ tends to a time-invariant random variable, namely $\log \left(k_{i t}\right)=\log \left(k_{i}\right)+\tau_{i t}$, where $\tau_{i t}$ is a stationary process representing all country-specific forces driving the country's business cycles (see, for instance, Lee et al., 1997). So we have:

$$
\tilde{y}_{i t}=a_{i 0}+\alpha_{i} \log \left(k_{i}\right)+g_{i} t+\gamma_{i} a_{t}+e_{i t}+\tau_{i t} .
$$

Taking first differences we obtain,

$$
\Delta \tilde{y}_{i t}=\tilde{g}_{i}+\gamma_{i} f_{t}+\varepsilon_{i t}
$$

where $f_{t}=\Delta a_{t}=a_{t}-a_{t-1}$, and $\varepsilon_{i t}=\Delta e_{i t}+\Delta \tau_{i t}$. In terms of $\log$ output, $y_{i t}=\ln \left(Y_{i t}\right)$, we now obtain equation (1), with $\Delta y_{i t}=y_{i t}-y_{i, t-1}, g_{i}=\tilde{g}_{i}+n_{i}$.

\section{A.2 Country-specific Equity Excess Return}

We first note that using (20) in (18) and (19) we obtain

$$
E_{t}\left(\Delta y_{i, t+j+1}\right)=g_{i}+\gamma_{i} \phi^{j+1} f_{t}
$$


and

$$
\begin{aligned}
E_{t}\left(r_{t+j+1}^{f}\right) & \approx r+\varrho g+\left(\gamma \varrho \phi_{f}^{j+1}\right) f_{t}-\frac{1}{2}\left(\varrho^{2} \gamma^{2} b_{f}\right)\left(\frac{\left(1-b_{f}^{j}\right) a_{f}}{1-b_{f}}+b_{f}^{j} \nu_{t}^{2}\right)+O\left(N^{-1}\right) \\
& =r+\varrho g-\frac{1}{2} \frac{\left(1-b_{f}^{j}\right)\left(\varrho^{2} \gamma^{2} b_{f}\right) a_{f}}{1-b_{f}}+\left(\gamma \varrho \phi_{f}^{j+1}\right) f_{t}-\frac{1}{2} \varrho^{2} \gamma^{2} b_{f}^{j+1} \nu_{t}^{2}+O\left(N^{-1}\right)
\end{aligned}
$$

Substituting the above results in (17) we have

$$
\begin{aligned}
\delta_{i t} & =\sum_{j=0}^{\infty} \kappa_{i}^{j}\left[r+\varrho g-\frac{1}{2} \frac{\left(1-b_{f}^{j}\right)\left(\varrho^{2} \gamma^{2} b_{f}\right) a_{f}}{1-b_{f}}+\left(\gamma \varrho \phi_{f}^{j+1}\right) f_{t}-\frac{1}{2} \varrho^{2} \gamma^{2} b_{f}^{j+1} \nu_{t}^{2}-g_{i}-\gamma_{i} \phi_{f}^{j+1} f_{t}\right]+O\left(N^{-1}\right) \\
& =\frac{r+\varrho g-g_{i}-\frac{1}{2} \frac{\left(\varrho^{2} \gamma^{2} b_{f}\right) a_{f}}{1-b_{f}}}{1-\kappa_{i}}+\frac{1}{2} \frac{\left(\varrho^{2} \gamma^{2} b_{f}\right) a_{f}}{\left(1-b_{f}\right)\left(1-b_{f} \kappa_{i}\right)}+\left(\frac{\left(\gamma \varrho-\gamma_{i}\right) \phi_{f}}{1-\phi_{f} \kappa_{i}}\right) f_{t}-\frac{1}{2} \frac{\varrho^{2} \gamma^{2} b_{f}}{\left(1-b_{f} \kappa_{i}\right)} \nu_{t}^{2}+O\left(N^{-1}\right)
\end{aligned}
$$

which if used in (16), and after some algebra, yields

$$
r_{i, t+1}=a_{i}+\varepsilon_{i, t+1}+\frac{\left(\gamma \varrho-\gamma_{i}\right) \phi_{f}}{1-\phi_{f} \kappa_{i}} f_{t}+\left(\frac{\gamma_{i}-\kappa_{i} \gamma \varrho \phi_{f}}{1-\phi_{f} \kappa_{i}}\right) f_{t+1}-\frac{1}{2}\left(\frac{\varrho^{2} \gamma^{2} b_{f}}{1-b_{f} \kappa_{i}}\right)\left(\nu_{t}^{2}-\kappa_{i} \nu_{t+1}^{2}\right)
$$

where

$$
a_{i}=r+\varrho g-\frac{1}{2} \frac{\kappa_{i} \varrho^{2} \gamma^{2} b_{f} a_{f}}{1-b_{f} \kappa_{i}} .
$$

It is also helpful to note that (A.2) can be written equivalently as

$$
\begin{aligned}
r_{i, t+1}= & r+\varrho g+\varepsilon_{i, t+1}+\gamma \varrho \phi_{f} f_{t}-\frac{1}{2} \varrho^{2} \gamma^{2} b_{f} \nu_{t}^{2}+\left(\frac{\gamma_{i}-\kappa_{i} \gamma \varrho \phi_{f}}{1-\phi_{f} \kappa_{i}}\right)\left(f_{t+1}-\phi_{f} f_{t}\right) \\
& +\frac{1}{2}\left(\frac{\varrho^{2} \gamma^{2} b_{f} \kappa_{i}}{1-b_{f} \kappa_{i}}\right)\left(\nu_{t+1}^{2}-a_{f}-b_{f} \nu_{t}^{2}\right)+O\left(N^{-1}\right)
\end{aligned}
$$

Subtracting $r_{t+1}^{f}$ from both sides of (A.3), using the equation for the risk free rate given by (15), we also obtain the following expression for country-specific excess returns

$r_{i, t+1}-r_{t+1}^{f}=\frac{1}{2} \varrho^{2} \gamma^{2} a_{f}+\left(\frac{\gamma_{i}-\kappa_{i} \gamma \varrho \phi_{f}}{1-\phi_{f} \kappa_{i}}\right) \nu_{t+1}+\frac{1}{2}\left(\frac{\varrho^{2} \gamma^{2} b_{f} \kappa_{i}}{1-b_{f} \kappa_{i}}\right)\left(\nu_{t+1}^{2}-a_{f}-b_{f} \nu_{t}^{2}\right)+\varepsilon_{i, t+1}+O\left(N^{-1}\right)$,

which yields $E_{t}\left(r_{i, t+1}-r_{t+1}^{f}\right)=\frac{1}{2} \varrho^{2} \gamma^{2} a_{f}+O\left(N^{-1}\right)$. Therefore, in our multi-country model with complete markets, country-specific risk gets diversified completely, and excess return predictability only arises if $N$, the number of countries participating in global risk sharing, is not large enough. However, there is still a non-zero risk premium for equity holdings so long as $\varrho^{2}>0$. Recall that $a_{f}>0, \gamma^{2}>0$. 


\section{A.3 Proof of Proposition 4 (Consistent estimation of factors in a dynamic heterogeneous multi-country model)}

Proof. Using the country-specific models given by (54), and solving for $\mathbf{z}_{i t}$ in terms of current and past values of factors and shocks we have:

$$
\mathbf{z}_{i t}=\boldsymbol{\mu}_{i}+\sum_{\ell=0}^{\infty} \boldsymbol{\Phi}_{i}^{\ell} \boldsymbol{\Gamma}_{i} \mathbf{f}_{t-\ell}+\varkappa_{i t}
$$

where

$$
\boldsymbol{\mu}_{i}=\left(\mathbf{I}_{2}-\boldsymbol{\Phi}_{i}\right)^{-1} \mathbf{a}_{i}, \varkappa_{i t}=\sum_{\ell=0}^{\infty} \boldsymbol{\Phi}_{i}^{\ell} \boldsymbol{\vartheta}_{i, t-\ell}, \text { and } \boldsymbol{\vartheta}_{i t}=\left(\eta_{i t}, \varepsilon_{i t}\right)^{\prime} .
$$

Assumption 8, ensures that the infinite sums are convergent. Pre-multiplying both sides of (A.5) by $\left(w_{i}\right)$ and summing over $i$ yields:

$$
\overline{\mathbf{z}}_{\omega t}=\overline{\boldsymbol{\mu}}_{\omega}+\sum_{\ell=0}^{\infty} \mathbf{A}_{\ell, N} \mathbf{f}_{t-\ell}+\bar{\varkappa}_{\omega t}
$$

where

$$
\begin{aligned}
& \overline{\mathbf{z}}_{\omega t}=\sum_{i=1}^{N} w_{i} \mathbf{z}_{i t}, \quad \overline{\boldsymbol{\mu}}_{\omega}=\sum_{i=1}^{N} w_{i} \boldsymbol{\mu}_{i}, \\
& \mathbf{A}_{\ell, N}=\sum_{i=1}^{N} w_{i} \boldsymbol{\Phi}_{i}^{\ell} \boldsymbol{\Gamma}_{i}, \text { and } \bar{\varkappa}_{\omega t}=\sum_{i=1}^{N} w_{i} \varkappa_{i t} .
\end{aligned}
$$

Under Assumption $5, \varkappa_{i t}$ are cross-sectionally weakly correlated and the weights $\mathbf{w}=\left(w_{1}, w_{2}, \ldots, w_{N}\right)^{\prime}$ are granular. By results in Pesaran and Chudik (2014), it readily follows that:

$$
\bar{\varkappa}_{\omega t}=O(\|\mathbf{w}\|)=O\left(N^{-1 / 2}\right) \text {, for each } t
$$

Under Assumptions 7 and 8, we also have

$$
\mathbb{E}\left(\boldsymbol{\Phi}_{i}^{\ell} \boldsymbol{\Gamma}_{i}\right)=\mathbb{E}\left(\boldsymbol{\Phi}_{i}^{\ell}\right) \mathbb{E}\left(\boldsymbol{\Gamma}_{i}\right)=\boldsymbol{\Lambda}_{\ell} \boldsymbol{\Gamma},
$$

and since $\boldsymbol{\Phi}_{i}$ and $\boldsymbol{\Gamma}_{i}$ are distributed independently across $i$, using again results in Pesaran and Chudik (2014) we have:

$$
\mathbf{A}_{\ell, N}-\mathbb{E}\left(\mathbf{A}_{\ell, N}\right)=\sum_{i=1}^{N} w_{i}\left[\boldsymbol{\Phi}_{i}^{\ell} \boldsymbol{\Gamma}_{i}-\mathbb{E}\left(\boldsymbol{\Phi}_{i}^{\ell} \boldsymbol{\Gamma}_{i}\right)\right]=O(\|\mathbf{w}\|)=O\left(N^{-1 / 2}\right) .
$$


Using (A.9) and (A.10) in (A.7) we now have:

$$
\begin{aligned}
\overline{\mathbf{z}}_{\omega t} & =\overline{\boldsymbol{\mu}}_{\omega}+\sum_{\ell=0}^{\infty} \boldsymbol{\Lambda}_{\ell} \boldsymbol{\Gamma} \boldsymbol{f}_{t-\ell}+O_{p}\left(N^{-1 / 2}\right) \\
& =\overline{\boldsymbol{\mu}}_{\omega}+\left(\sum_{\ell=0}^{\infty} \boldsymbol{\Lambda}_{\ell} L^{\ell}\right) \boldsymbol{\Gamma} \boldsymbol{f}_{t}+O_{p}\left(N^{-1 / 2}\right) \\
& =\overline{\boldsymbol{\mu}}_{\omega}+\boldsymbol{\Lambda}(L) \boldsymbol{\Gamma} \mathbf{f}_{t}+O_{p}\left(N^{-1 / 2}\right) .
\end{aligned}
$$

But under Assumptions 7 and $8, \boldsymbol{\Gamma}$ and $\boldsymbol{\Lambda}(L)$ are both invertible and:

$$
\mathbf{f}_{t}=\boldsymbol{\Gamma}^{-1} \boldsymbol{\Lambda}^{-1}(L)\left(\overline{\mathbf{z}}_{\omega t}-\overline{\boldsymbol{\mu}}_{\omega}\right)+O_{p}\left(N^{-1 / 2}\right)
$$

where:

$$
\begin{aligned}
\boldsymbol{\Gamma}^{-1} & =\left(\begin{array}{cc}
0 & \gamma^{-1} \\
\theta^{-1} & -\frac{\lambda}{\theta \gamma}
\end{array}\right) \\
\boldsymbol{\Lambda}^{-1}(L) & =\mathbf{B}_{0}+\mathbf{B}_{1} L+\mathbf{B}_{2} L^{2}+\ldots
\end{aligned}
$$

(note that $\mathbf{B}_{0}=\boldsymbol{\Lambda}_{0}=\mathbf{I}_{2}$ ). Hence,

$$
\begin{aligned}
\mathbf{f}_{t} & =\boldsymbol{\Gamma}^{-1}\left(\overline{\mathbf{z}}_{\omega t}-\overline{\boldsymbol{\mu}}_{\omega}\right)+\left(\mathbf{C}_{1}+\mathbf{C}_{2} L+\mathbf{C}_{3} L^{2}+\ldots .\right)\left(\overline{\mathbf{z}}_{\omega, t-1}-\overline{\boldsymbol{\mu}}_{\omega}\right)+O_{p}\left(N^{-1 / 2}\right) \\
& =\mathbf{b}+\left(\sum_{\ell=0}^{\infty} \mathbf{C}_{\ell} L^{\ell}\right) \overline{\mathbf{z}}_{\omega, t}+O_{p}\left(N^{-1 / 2}\right)
\end{aligned}
$$

where $\mathbf{C}_{\ell}=\boldsymbol{\Gamma}^{-1} \mathbf{B}_{\ell}$, for $\ell=0,1,2, \ldots$, and $\mathbf{b}=-\boldsymbol{\Gamma}^{-1} \boldsymbol{\Lambda}^{-1}(1) \overline{\boldsymbol{\mu}}_{\omega}$. But given the lower triangular form of $\boldsymbol{\Gamma}^{-1}$, we have

$$
\begin{aligned}
& f_{t}=\gamma^{-1} \Delta \bar{y}_{\omega, t}+\sum_{\ell=1}^{\infty} \mathbf{c}_{1, \ell}^{\prime} \overline{\mathbf{z}}_{\omega, t-\ell}+O_{p}\left(N^{-1 / 2}\right) \\
& g_{t}=\theta^{-1} \bar{v}_{\omega, t}-\left(\frac{\lambda}{\theta \gamma}\right) \Delta \bar{y}_{\omega, t}+\sum_{\ell=1}^{\infty} \mathbf{c}_{2, \ell}^{\prime} \overline{\mathbf{z}}_{\omega, t-\ell}+O_{p}\left(N^{-1 / 2}\right)
\end{aligned}
$$

where $\mathbf{c}_{1, \ell}^{\prime}$ and $\mathbf{c}_{2, \ell}^{\prime}$ are the first and the second rows of $\mathbf{C}_{\ell}$, respectively, and $\bar{v}_{\omega, t}, \Delta \bar{y}_{\omega, t}, \overline{\mathbf{z}}_{\omega, t}$ are defined as above.

Consider now $\mathbf{C}_{\ell}$ and note that $\left\|\mathbf{C}_{\ell}\right\| \leq\left\|\boldsymbol{\Gamma}^{-1}\right\|\left\|\mathbf{B}_{\ell}\right\|$, where $\left\|\boldsymbol{\Gamma}^{-1}\right\|$ is bounded for fixed 
non-zero values of $\gamma$ and $\theta$. Further, $\mathbf{B}_{\ell}$ is given by the following recursions

$$
\begin{aligned}
\mathbf{B}_{0}= & \mathbf{I}_{2}, \mathbf{B}_{1}=-\boldsymbol{\Lambda}_{1} \\
\mathbf{B}_{2}= & -\left(\boldsymbol{\Lambda}_{1} \mathbf{B}_{1}+\boldsymbol{\Lambda}_{2} \mathbf{B}_{0}\right) \\
& \vdots \\
\mathbf{B}_{\ell}= & -\left(\boldsymbol{\Lambda}_{1} \mathbf{B}_{\ell-1}+\boldsymbol{\Lambda}_{2} \mathbf{B}_{\ell-1}+\ldots .+\boldsymbol{\Lambda}_{\ell} \mathbf{B}_{0}\right) .
\end{aligned}
$$

Hence, $\left\|\mathbf{B}_{1}\right\| \leq\left\|\boldsymbol{\Lambda}_{1}\right\|\left\|\mathbf{B}_{0}\right\|,\left\|\mathbf{B}_{2}\right\| \leq\left\|\boldsymbol{\Lambda}_{1}\right\|\left\|\mathbf{B}_{1}\right\|+\left\|\boldsymbol{\Lambda}_{2}\right\|\left\|\mathbf{B}_{0}\right\|$, and in general $\left\|\mathbf{B}_{\ell}\right\| \leq\left\|\boldsymbol{\Lambda}_{1}\right\|\left\|\mathbf{B}_{\ell-1}\right\|+$ $\left\|\boldsymbol{\Lambda}_{2}\right\|\left\|\mathbf{B}_{\ell-1}\right\|+\ldots .+\left\|\boldsymbol{\Lambda}_{\ell}\right\|\left\|\mathbf{B}_{0}\right\|$, where $\left\|\mathbf{B}_{0}\right\|=1$. However,

$$
\left\|\boldsymbol{\Lambda}_{\ell}\right\|=\left\|\mathbb{E}\left(\boldsymbol{\Phi}_{i}^{\ell}\right)\right\| \leq \mathbb{E}\left\|\boldsymbol{\Phi}_{i}^{\ell}\right\| \leq\left(\mathbb{E}\left\|\boldsymbol{\Phi}_{i}\right\|\right)^{\ell} \leq \rho^{\ell} .
$$

Hence, $\left\|\mathbf{B}_{1}\right\| \leq \rho,\left\|\mathbf{B}_{2}\right\| \leq \rho^{2}$, and so on, and as required $\left\|\mathbf{C}_{\ell}\right\| \leq\left\|\boldsymbol{\Gamma}^{-1}\right\| \rho^{\ell} .49$

\section{A.4 Proof of Proposition 5 (Consistent estimation of the orthonormalized factors in the dynamic case)}

Proof. Consider equation (58) and (59) in the main text. Let $M_{\bar{Z}_{\omega}}=\mathbf{I}_{T}-\overline{\mathbf{Z}}_{\omega}\left(\overline{\mathbf{Z}}_{\omega}^{\prime} \overline{\mathbf{Z}}_{\omega}\right)^{-1} \overline{\mathbf{Z}}_{\omega}^{\prime}$, and note that:

$$
\begin{aligned}
\mathbf{M}_{\bar{Z}_{\omega}} \mathbf{f} & =\mathbf{M}_{\bar{Z}_{\omega}} \Delta \overline{\mathbf{y}}_{\omega} \\
\mathbf{M}_{\bar{Z}_{\omega}} \mathbf{g} & =\mathbf{M}_{\bar{Z}_{\omega}} \overline{\mathbf{v}}_{\omega}-\lambda \mathbf{M}_{\bar{Z}_{\omega}} \Delta \overline{\mathbf{y}}_{\omega}
\end{aligned}
$$

since $\mathbf{M}_{\bar{Z}_{\omega}} \overline{\mathbf{Z}}_{\omega}=\mathbf{0}$. We set the first normalized vector of innovations, denoted by $\hat{\boldsymbol{\zeta}}$, to $\mathbf{M}_{\bar{Z}_{\omega}} \mathbf{f}$, namely $\hat{\boldsymbol{\zeta}}=\mathbf{M}_{\bar{Z}_{\omega}} \Delta \overline{\mathbf{y}}_{\omega}$, and set the second factor, that we label $\hat{\boldsymbol{\xi}}$, as the linear combination of $\mathbf{M}_{\bar{Z}_{\omega}} \mathbf{f}$ and $\mathbf{M}_{\bar{Z}_{\omega}} \mathbf{g}$ such that $\hat{\boldsymbol{\zeta}}^{\prime} \hat{\boldsymbol{\xi}}=0$. This can be achieved selecting $\lambda$ so that:

$$
\hat{\zeta}^{\prime} \hat{\boldsymbol{\xi}}=\Delta \overline{\mathbf{y}}_{\omega}^{\prime} \mathbf{M}_{\bar{Z}_{\omega}}\left(\mathbf{M}_{\bar{Z}_{\omega}} \overline{\mathbf{v}}_{\omega}-\lambda \mathbf{M}_{\bar{Z}_{\omega}} \Delta \overline{\mathbf{y}}_{\omega}\right)=0
$$

The value of $\lambda$ that solves this equation is given by:

$$
\hat{\lambda}=\frac{\Delta \overline{\mathbf{y}}_{\omega}^{\prime} \mathbf{M}_{\bar{Z}_{\omega}} \overline{\mathbf{v}}_{\omega}}{\Delta \overline{\mathbf{y}}_{\omega}^{\prime} \mathbf{M}_{\bar{Z}_{\omega}} \Delta \overline{\mathbf{y}}_{\omega}} .
$$

Note that $\hat{\lambda}$ is the OLS estimator of the coefficient of the regression of $\mathbf{M}_{\bar{Z}_{\omega}} \overline{\mathbf{v}}_{\omega}$ on $\mathbf{M}_{\bar{Z}_{\omega}} \Delta \overline{\mathbf{y}}_{\omega}$. Hence, the orthogonalized factors are

$$
\begin{aligned}
\hat{\boldsymbol{\zeta}} & =\mathbf{M}_{\bar{Z}_{\omega}} \Delta \overline{\mathbf{y}}_{\omega}, \\
\hat{\boldsymbol{\xi}} & =\mathbf{M}_{\bar{Z}_{\omega}} \overline{\mathbf{v}}_{\omega}-\hat{\lambda} \mathbf{M}_{\bar{Z}_{\omega}} \Delta \overline{\mathbf{y}}_{\omega} .
\end{aligned}
$$

\footnotetext{
${ }^{49}$ Note that for any matrix $\mathbf{A},\left\|\mathbf{A}^{p}\right\| \leq\|\mathbf{A}\|^{p}$, and for any random variable $X,\|E(X)\| \leq E\|X\|$.
} 
In practice, this implies that $\hat{\zeta}$ can be recovered as residuals from the OLS regression of $\Delta \overline{\mathbf{y}}_{\omega}$ on an intercept and $\overline{\mathbf{z}}_{\omega, t-\ell}$, for $\ell=1,2, \ldots, p$ :

$$
\Delta \overline{\mathbf{y}}_{\omega}=\overline{\mathbf{Z}}_{\omega} \hat{\mathbf{c}}_{1}+\hat{\boldsymbol{\zeta}}
$$

While $\hat{\boldsymbol{\xi}}$ can be recovered as residuals from the OLS regression of $\overline{\mathbf{v}}_{\omega}$ on $\hat{\boldsymbol{\zeta}}$, an intercept, and $\overline{\mathbf{z}}_{\omega, t-\ell}$, for $\ell=1, \ldots, p$ :

$$
\overline{\mathbf{v}}_{\omega}=\hat{\lambda} \hat{\boldsymbol{\zeta}}+\overline{\mathbf{Z}}_{\omega} \hat{\mathbf{c}}_{2}+\hat{\boldsymbol{\xi}}
$$




\section{B Data Sources}

For equity prices we use the MSCI Index (excluding dividends) in local currency. We collected daily observations from January 1979 to June 2011, but the panel of countries is unbalanced with only 16 economies starting from the beginning of the sample. A balanced panel was also constructed with 32 countries from 1993:Q1. The data source for the daily equity price indices is Bloomberg. The countries included in the sample are the following: Argentina, Australia, Austria, Belgium, Brazil, Canada, Chile, China, Finland, France, Germany, India, Indonesia, Italy, Japan, Korea, Malaysia, Mexico, Netherlands, Norway, New Zealand, Peru, Philippines, Saudi Arabia, South Africa, Singapore, Spain, Sweden, Switzerland, Thailand, Turkey, United Kingdom, and United States.

The list of Bloomberg tickers is as follows: MSELTAG, MSDLAS, MSDLAT, MSDLBE, MSELTBR, MSDLCA, MSELTCF, MSELTCH, MSDLFI, MSDLFR, MSDLGR, MSELTIA, MSELTINF, MSDLIT, MSDLJN, MSELTKO, MXMY, MSELTMXF , MSDLNE, MSDLNO, MSDLNZ, MSELTPR, MSELTPHF, MSELTSA, MGCLSA, MSDLSG, MSDLSP, MSDLSW, MSDLSZ, MSELTTHF, MSELTTK, MSDLUK, MSDLUS.

Real GDP data come from standard sources. The data set is balanced and good quality quarterly data are also available for all countries from 1993:Q1. For more details see:

https://sites.google.com/site/gvarmodelling/. 


\section{Online Supplement to 'Uncertainty and Economic Activity: A Multi-Country Perspective'}

by A. Cesa-Bianchi, and M.Hashem Pesaran, and A. Rebucci

February 7, 2018 


\section{S1 Realized Volatility versus Cross-sectional Dispersion}

As noted in the paper, if we consider a panel of country equities (e.g., of firms or sectors within a country), a different measure of uncertainty can be computed as the cross-sectional dispersion of equity prices. In this section we show that this concept is closely related to the realized volatility measures we consider. To illustrate the point with the data that we use in our application, we derive results at the 'country-specific versus world level' rather than 'firm-specific versus country level'. ${ }^{50}$ Specifically, we compare the cross-sectional dispersion of equity returns across countries with the realized volatility of 'world' equity returns.

Define the daily cross-country dispersion of equity returns as:

$$
\sigma_{c d t}=\sqrt{D_{t}^{-1} \sum_{\tau=1}^{D_{t}} \sum_{i=1}^{N} w_{i}\left(r_{i t}(\tau)-\bar{r}_{t}(\tau)\right)^{2}}
$$

and the daily realized volatility of world equity returns as:

$$
\sigma_{r v t}=\sqrt{D_{t}^{-1} \sum_{i=1}^{N} \sum_{\tau=1}^{D_{t}} w_{i t}\left(r_{i t}(\tau)-\bar{r}_{i t}\right)^{2}}
$$

where $r_{i t}(\tau)=\Delta \ln P_{i t}(\tau)$ and $\bar{r}_{i t}=D_{t}^{-1} \sum_{\tau=1}^{D_{t}} r_{i t}(\tau)$ is the average daily price change over the quarter $t$, and $D_{t}$ is the number of trading days in quarter $t$; and $w_{i}$ is the weight attached to country $i$. To establish the relation between these two measures it is easier to work with their squares:

$$
\begin{aligned}
\sigma_{r v t}^{2} & =D_{t}^{-1} \sum_{i=1}^{N} \sum_{\tau=1}^{D_{t}} w_{i}\left(r_{i t}(\tau)-\bar{r}_{i t}\right)^{2}, \\
\sigma_{c d t}^{2} & =D_{t}^{-1} \sum_{\tau=1}^{D_{t}} \sum_{i=1}^{N} w_{i}\left(r_{i t}(\tau)-\bar{r}_{t}(\tau)\right)^{2} .
\end{aligned}
$$

Note also that

$$
\sigma_{r v t}^{2}=D_{t}^{-1} \sum_{i=1}^{N} \sum_{\tau=1}^{D_{t}} w_{i} r_{i t}^{2}(\tau)-\sum_{i=1}^{N} w_{i} \bar{r}_{i t}^{2}
$$

and

$$
\sigma_{c d t}^{2}=D_{t}^{-1} \sum_{\tau=1}^{D_{t}} \sum_{i=1}^{N} w_{i} r_{i t}^{2}(\tau)-\sum_{i=1}^{N} w_{i}\left(D_{t}^{-1} \sum_{\tau=1}^{D_{t}} \bar{r}_{t}^{2}(\tau)\right) .
$$

\footnotetext{
${ }^{50}$ Our analysis holds at the firm-specific versus country level as well.
} 
Hence, since $\sum_{i=1}^{N} w_{i}=1$, it follows that

$$
\sigma_{c d t}^{2}-\sigma_{r v t}^{2}=\sum_{i=1}^{N} w_{i} \bar{r}_{i t}^{2}-D_{t}^{-1} \sum_{\tau=1}^{D_{t}} \bar{r}_{t}^{2}(\tau)
$$

where $\bar{r}_{i t}=D_{t}^{-1} \sum_{\tau=1}^{D_{t}} r_{i t}(\tau)$, and $\bar{r}_{t}(\tau)=\sum_{i=1}^{N} w_{i} r_{i t}(\tau)$.

Suppose now that daily returns have the following single-factor structure: ${ }^{51}$

$$
r_{i t}(\tau)=\beta_{i} f_{t}(\tau)+\varepsilon_{i t}(\tau)
$$

where the factor is strong in the sense that (see Bailey et al., 2016)

$$
\lim _{N \rightarrow \infty} \sum_{i=1}^{N} w_{i} \beta_{i}=\bar{\beta} \neq 0, \text { and } \lim _{N \rightarrow \infty} \sum_{i=1}^{N} w_{i} \beta_{i}^{2}=\sigma_{\beta}^{2}+\bar{\beta}^{2} \neq 0 .
$$

The idiosyncratic components, $\varepsilon_{i t}(\tau)$, are assumed to be independently distributed from $\beta_{i} f_{t}(\tau)$, cross-sectionally weakly correlated, and serially uncorrelated with zero means and finite variances. Also let:

$$
\lim _{D_{t} \rightarrow \infty} D_{t}^{-1} \sum_{\tau=1}^{D_{t}} f_{t}^{2}(\tau)=h_{f_{t}}^{2}
$$

We now note that

$$
\begin{aligned}
\sum_{i=1}^{N} w_{i} \bar{r}_{i t}^{2} & =\left(\sum_{i=1}^{N} w_{i} \beta_{i}^{2}\right) \bar{f}_{t}^{2}+\left(\sum_{i=1}^{N} w_{i} \bar{\varepsilon}_{i t}^{2}\right)+2\left(\sum_{i=1}^{N} w_{i} \beta_{i} \bar{\varepsilon}_{i t}\right) \bar{f}_{t} \\
& =\left(\sigma_{\beta}^{2}+\bar{\beta}^{2}\right) \bar{f}_{t}^{2}+O_{p}\left(D_{t}^{-1 / 2}\right)+O_{p}\left(N^{-1 / 2}\right),
\end{aligned}
$$

where $\bar{f}_{t}=D_{t}^{-1} \sum_{\tau=1}^{D_{t}} f_{t}(\tau)$, and $\bar{\varepsilon}_{i t}=D_{t}^{-1} \sum_{\tau=1}^{D_{t}} \varepsilon_{i t}(\tau)$. Also

$$
\begin{aligned}
D_{t}^{-1} \sum_{\tau=1}^{D_{t}} \bar{r}_{t}^{2}(\tau) & =D_{t}^{-1} \sum_{\tau=1}^{D_{t}}\left[\bar{\beta} f_{t}(\tau)+\bar{\varepsilon}_{t}(\tau)\right]^{2} \\
& =\bar{\beta}^{2}\left[D_{t}^{-1} \sum_{\tau=1}^{D_{t}} f_{t}^{2}(\tau)\right]+D_{t}^{-1} \sum_{\tau=1}^{D_{t}} \bar{\varepsilon}_{t}^{2}(\tau)+2 D_{t}^{-1} \sum_{\tau=1}^{D_{t}} \bar{\beta} \bar{\varepsilon}_{t}(\tau) f_{t}(\tau) \\
& =\bar{\beta}^{2} h_{f_{t}}^{2}+O_{p}\left(N^{-1 / 2}\right)+O_{p}\left(D_{t}^{-1 / 2}\right) .
\end{aligned}
$$

Hence

$$
\begin{aligned}
\sigma_{c d t}^{2}-\sigma_{r v t}^{2} & =\left(\sigma_{\beta}^{2}+\bar{\beta}^{2}\right) \bar{f}_{t}^{2}-\bar{\beta}^{2} h_{f_{t}}^{2}+O_{p}\left(N^{-1 / 2}\right)+O_{p}\left(D_{t}^{-1 / 2}\right) \\
& =\sigma_{\beta}^{2} \bar{f}_{t}^{2}-\bar{\beta}^{2} \sigma_{f_{t}}^{2}+O_{p}\left(N^{-1 / 2}\right)+O_{p}\left(D_{t}^{-1 / 2}\right)
\end{aligned}
$$

\footnotetext{
${ }^{51}$ The analysis readily extends to more general multiple factor settings.
} 
where $\sigma_{f_{t}}^{2}=\left(h_{f_{t}}^{2}-\bar{f}_{t}^{2}\right) \geq 0$, is the variance of the common factor. This expression shows that, under fairly general assumptions (and for $N$ and $D_{t}$ sufficiently large) we would expect the cross-sectional dispersion measure to be closely related to asset-specific measures of realized volatility when the factor loadings, $\beta_{i}$, are not too dispersed across countries. The results also show that the relative magnitudes of the cross section dispersion and realized volatility measures depend on the relative values of $\sigma_{\beta}^{2} \bar{f}_{t}^{2}$ and $\bar{\beta}^{2} \sigma_{f_{t}}^{2}$.

Figure S.1 Realized Volatility and Cross-Sectional Dispersion

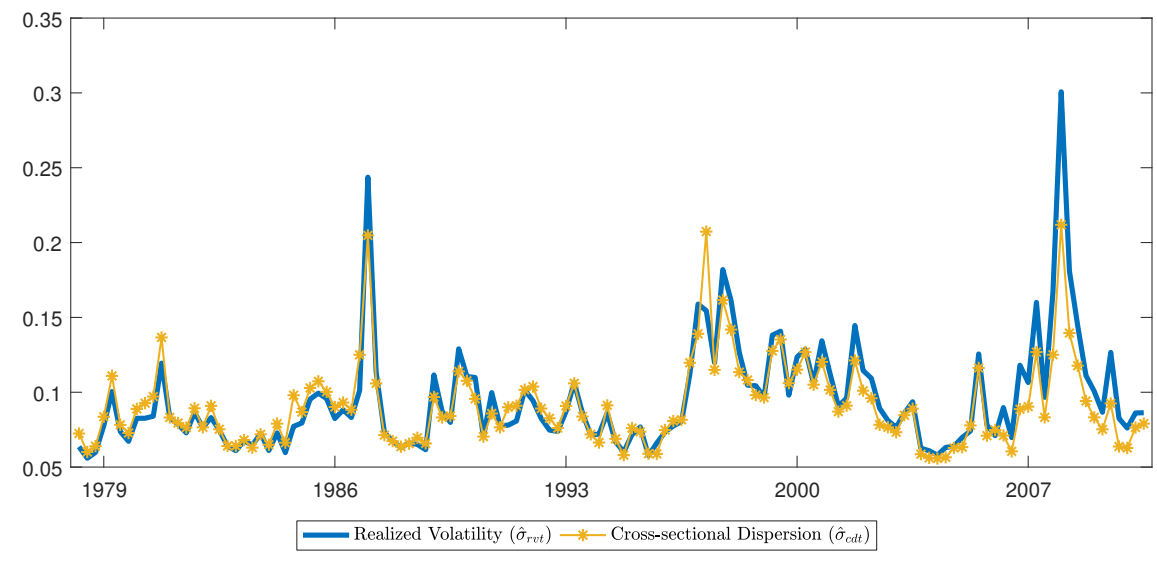

Note. World realized volatility of equity returns $\left(\hat{\sigma}_{r v t}\right)$ and cross-sectional dispersion of equity returns across countries $\left(\hat{\sigma}_{c d t}\right)$, computed as in equations (S1.2) and (S1.1), respectively. Both measures are expressed at quarterly rates and computed over the 1979:Q22011:Q2 period.

Figure S.1 compares world realized volatility $\left(\sigma_{r v t}\right.$, light thick line) and cross-sectional dispersion ( $\sigma_{c d t}$, dark thin line), computed as in equations (S1.2) and (S1.1), respectively, with equal weights. Their sample correlation over the 1979:Q1 to 2011:Q2 period is 0.92. Figure S.1 suggests that the two measures are very closely related, which is in line with the evidence provided by Bloom et al. (2012).

\section{S2 Comparison with Principal Components}

\section{S2.1 Principal Component versus Cross-sectional Averages}

In what follows we compare the cross-sectional average of the GDP growth rates $\left(\Delta \bar{y}_{\omega, t}\right)$ with the first principal component of the individual output growth series $\left\{\Delta y_{i t}, i=1,2, \ldots ., N ; t=1,2, \ldots ., T\right\}$, which we denote by $P C_{1, t}^{y}$. As can be seen from Figure S.2, the two series $\left(\Delta \bar{y}_{\omega, t}\right.$, and $\left.P C_{1, t}^{y}\right)$ move very closely and have a correlation coefficient of 0.9 . 


\section{S2.2 Principal Components of the Combined Panel of Output Growth Series and Realized Volatilities}

As stated in Remark 2 and 3 in the main text of the paper, applying principal component analysis to the panel of all volatility series or all volatility and growth series would not permit identifying $f_{t}$. To see this, we extract the first two principal components from the full panel of growth rates $\left(\Delta y_{i t}\right)$ and volatilities $\left(v_{i t}\right)$, which we label by $P C_{1, t}^{v y}$ and $P C_{2, t}^{v y}$, respectively. We then fit a $\operatorname{VAR}(1)$ model to these two PCs, and compute orthogonalized residuals from this VAR with a Cholesky decomposition placing the first PC, namely $P C_{1, t}^{v y}$, first (as discussed in

Section 3.3). We denote these orthogonalized residuals by $\epsilon^{P C_{1, t}^{v y}}$ and $\epsilon^{P C_{2, t}^{v y}}$. Figure S.3 plots $\epsilon^{P C_{1, t}^{v y}}$ together with $\hat{\zeta}_{t}$ (top panel) and $\epsilon^{P C_{2, t}^{v y}}$ together with $\hat{\xi}_{t}$ (bottom panel), and shows that the two series behave in a very different fashion, with correlations of -0.43 and 0.43 respectively.

\section{S2.3 Applying Principal Components Recursively}

While principal components extracted from the panel of all volatility and growth series do not permit identifying $f_{t}$, here we show how principal component analysis can be used to obtain estimates of the real and financial factors reported in the paper. As discussed in the paper, we need to follow a recursive procedure where the order of the recursion plays a crucial role. We need extract the first principal component from the panel of GDP growth rates, which as before we label by $P C_{1, t}^{y}$, and as noted earlier recovers a consistent estimator of $\zeta_{t}$ (up to an scalar). Next, we obtain the first principal component from the combined panel of output growth rates and volatilities and label it as $P C_{1, t}^{v y}$. Then, we estimate a $\operatorname{VAR}(1)$ in the principal components $\left(P C_{1, t}^{y}, P C_{1, t}^{v y}\right)^{\prime}$ to remove any serial correlation, and orthogonalize the residuals of this VAR with a Cholesky factorization of the variance-covariance matrix of this VAR's reduced form residuals, with $P C_{1, t}^{y}$ ordered first. Denote the resultant orthogonalized residuals by $\epsilon^{P C_{1, t}^{y}}$ and $\epsilon^{P C_{1, t}^{v y}}$.

Figure S.4 plots $\epsilon^{P C_{1, t}^{y}}$ together with $\hat{\zeta}_{t}$ (top panel) and $\epsilon^{P C_{1, t}^{v y}}$ together with $\hat{\xi}_{t}$ (bottom panel), and shows that they move in tandem, with correlations of 0.82 and 0.97 , respectively. Note, finally, that if we were to do a Cholesky factorization of the residuals from the same VAR but with the variables ordered in reverse, namely $\left(P C_{1, t}^{v y}, P C_{1, t}^{y},\right)^{\prime}$, the resultant orthogonalized residuals will not closely follow $\hat{\zeta}_{t}$ and $\hat{\xi}_{t}$. We conclude from this exercise that principal component analysis could be used to obtain qualitatively similar results that we report in the paper if applied in the recursive manner proposed here. 
Figure S.2 Estimating $\hat{\zeta}_{t}$ : Principal Component Versus

Cross-sectional Averages

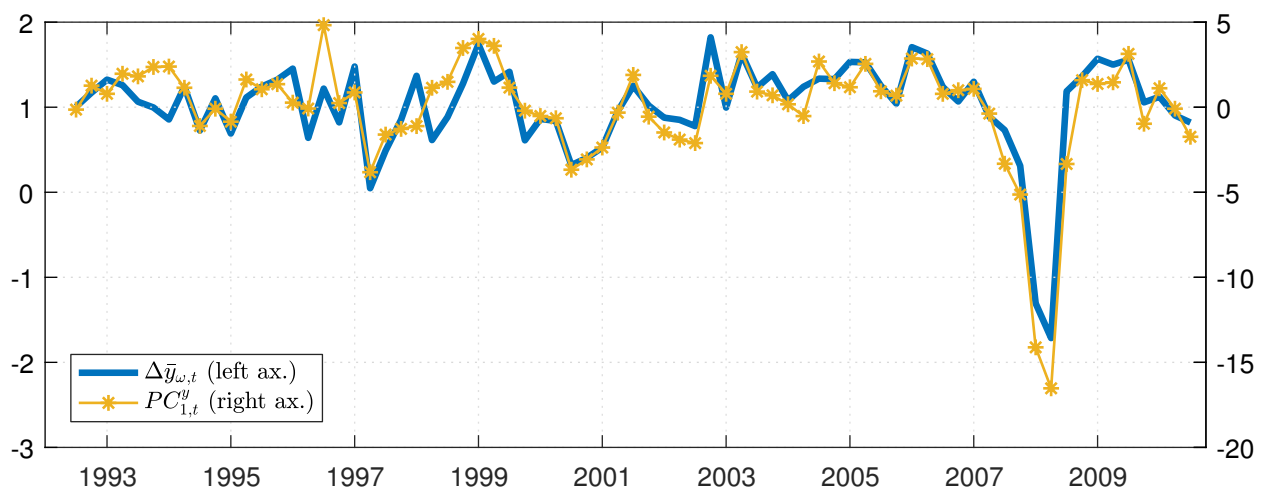

Note. Comparison of the cross-sectional average computed on the panel of GDP growth rates $\left(\Delta \bar{y}_{\omega, t}\right)$ and the first principal component computed on the same data set $\left(P C_{1, t}^{y}\right)$. Sample period: 1993:Q1-2011:Q2.

Figure S.3 Principal Component on the Full Panel of Volatility AND Growth SERIES
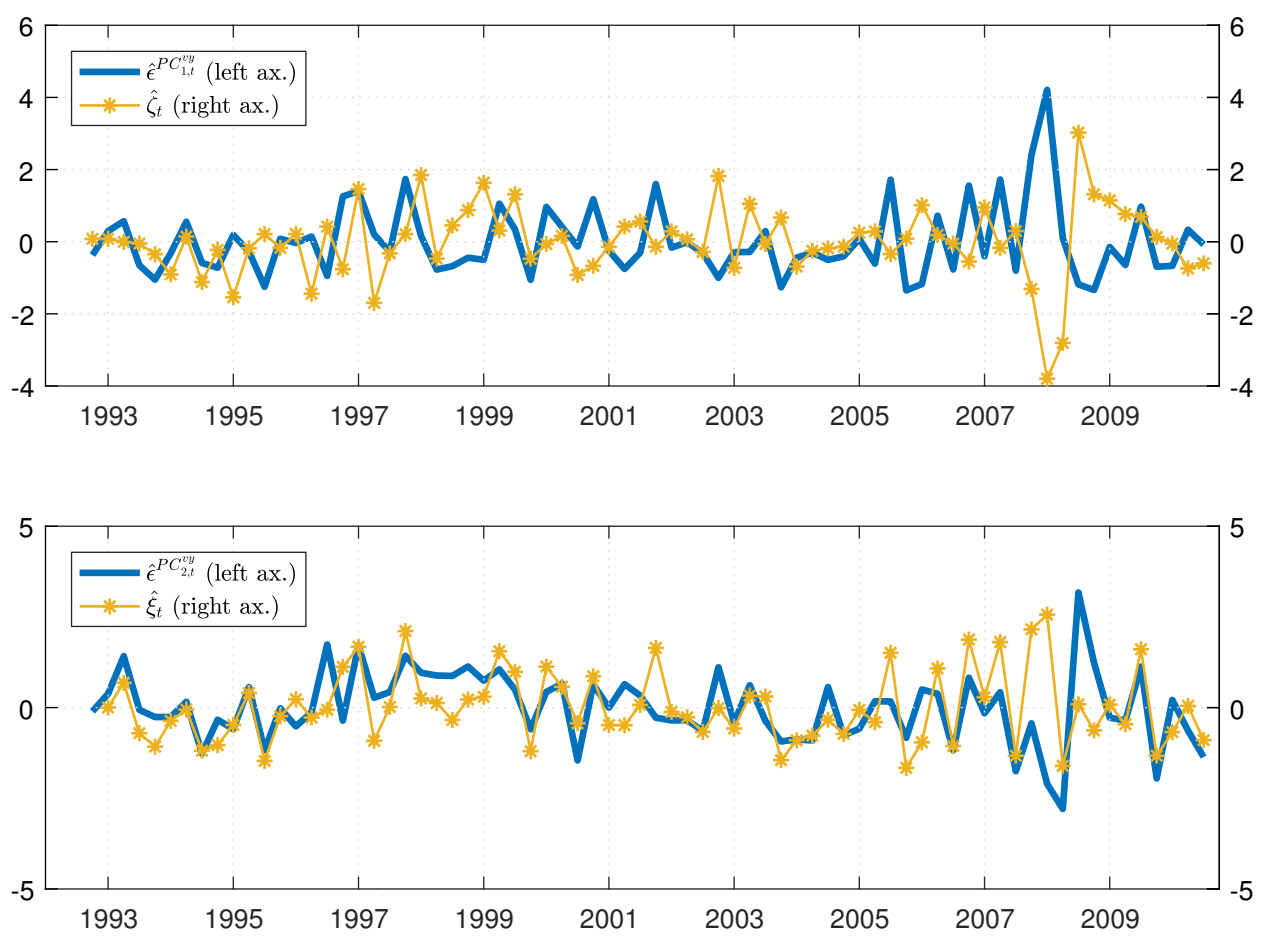

Note. Comparison of $\hat{\zeta}_{t}$ and $\hat{\xi}_{t}$ with the principal components obtained from the full panel of volatilities $\left(v_{i t}\right)$ and growth rates $\left(\Delta y_{i t}\right)$. Sample period: 1993:Q1-2011:Q2. 
Figure S.4 Principal Component of the Full Panel of Volatility and Growth Series Computed Recursively
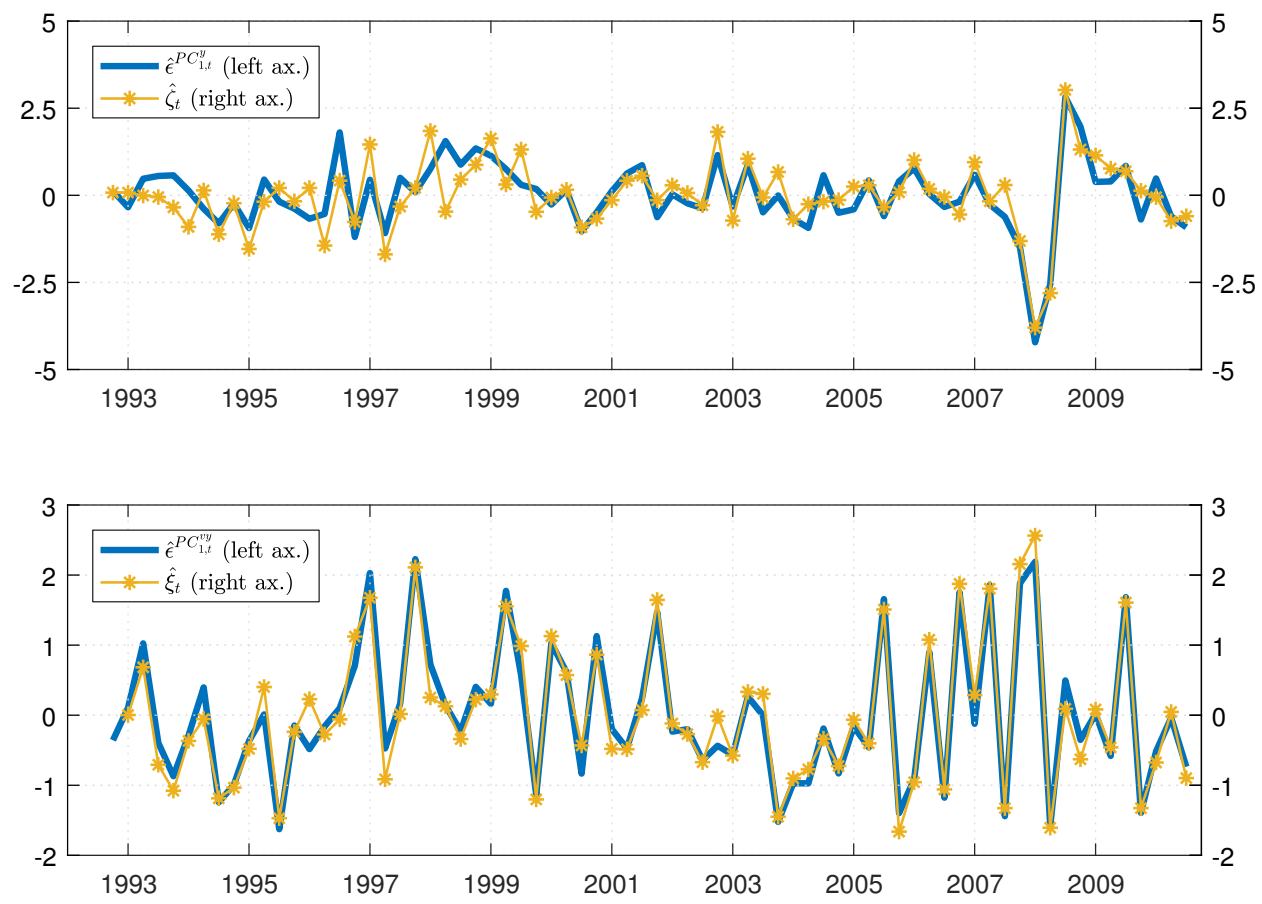

Note. Comparison of $\hat{\zeta}_{t}$ and $\hat{\xi}_{t}$ with the principal components obtained from the full panel of volatilities $\left(v_{i t}\right)$ and growth series $\left(\Delta y_{i t}\right)$ following the recursive procedure described in Section S2.3. Sample period: 1993:Q1-2011:Q2.

\section{S3 Computing Impulse Responses and Error Variance Decom- positions}

Consider the factor-augmented country-specific VAR models augmented with lagged cross section averages, $\overline{\mathbf{z}}_{\omega, t-\ell}$, for $\ell=1,2, \ldots, p$ as in equations (62)-(63) in the main text:

$$
\mathbf{z}_{i t}=\boldsymbol{\Phi}_{i} \mathbf{z}_{i, t-1}+\sum_{\ell=1}^{p} \boldsymbol{\psi}_{i, \ell} \overline{\mathbf{z}}_{\omega, t-\ell}+\boldsymbol{\beta}_{i} \boldsymbol{v}_{t}+\boldsymbol{\vartheta}_{i t}, \text { for } i=1,2, \ldots, N
$$

where:

$$
\boldsymbol{\beta}_{i}=\left(\begin{array}{cc}
\beta_{i, 11} & \beta_{i, 12} \\
\beta_{i, 21} & 0
\end{array}\right), \boldsymbol{v}_{t}=\left(\begin{array}{c}
\zeta_{t} \\
\xi_{t}
\end{array}\right)
$$

Intercepts are omitted to simplify the exposition. Note also that $\overline{\mathbf{z}}_{\omega, t}=\sum_{i=1}^{N} w_{i} \Delta \mathbf{z}_{i t}=\mathbf{W} \mathbf{z}_{t}$, where $\mathbf{z}_{t}=\left(\mathbf{z}_{1 t}^{\prime}, \mathbf{z}_{2 t}^{\prime}, \ldots, \mathbf{z}_{N t}^{\prime}\right)^{\prime}$, and $\mathbf{W}$ is a $2 \times 2 N$ matrix of weights. Stacking the VARs in (S3.1) over $i$ we obtain:

$$
\mathbf{z}_{t}=\mathbf{\Phi} \mathbf{z}_{t-1}+\sum_{\ell=1}^{p} \boldsymbol{\psi}_{\ell} \mathbf{W} \mathbf{z}_{t-\ell}+\boldsymbol{\beta} \boldsymbol{v}_{t}+\boldsymbol{\vartheta}_{t}
$$


where $\boldsymbol{\vartheta}_{t}=\left(\boldsymbol{\vartheta}_{1 t}^{\prime}, \boldsymbol{\vartheta}_{2 t}^{\prime}, \ldots, \boldsymbol{\vartheta}_{N t}^{\prime}\right)^{\prime}$ and:

$$
\boldsymbol{\Phi}=\left(\begin{array}{cccc}
\boldsymbol{\Phi}_{1} & \mathbf{0} & \cdots & \mathbf{0} \\
\mathbf{0} & \boldsymbol{\Phi}_{2} & \cdots & \mathbf{0} \\
\vdots & \vdots & \cdots & \vdots \\
\mathbf{0} & \mathbf{0} & \cdots & \boldsymbol{\Phi}_{N}
\end{array}\right), \boldsymbol{\psi}_{\ell}=\left(\begin{array}{c}
\psi_{1, \ell} \\
\psi_{2, \ell} \\
\vdots \\
\boldsymbol{\psi}_{N, \ell}
\end{array}\right), \quad \boldsymbol{\beta}=\left(\begin{array}{c}
\boldsymbol{\beta}_{1} \\
\boldsymbol{\beta}_{2} \\
\vdots \\
\boldsymbol{\beta}_{N}
\end{array}\right)
$$

The high-dimensional VAR in (S3.2) can now be written as a standard FAVAR $(p)$ model in $2 N$ variables:

$$
\mathbf{z}_{t}=\left(\boldsymbol{\Phi}+\boldsymbol{\psi}_{\mathbf{1}} \mathbf{W}\right) \mathbf{z}_{t-1}+\sum_{\ell=2}^{p} \boldsymbol{\psi}_{\ell} \mathbf{W} \mathbf{z}_{t-\ell}+\boldsymbol{\beta} \boldsymbol{v}_{t}+\boldsymbol{\vartheta}_{t}
$$

For example, when $p=1$ we have the $\operatorname{FAVAR}(1)$ :

$$
\mathbf{z}_{t}=\left(\mathbf{I}_{2 N}-\mathbf{\Psi}_{1} \mathbf{L}\right)^{-1}\left(\boldsymbol{\beta} \boldsymbol{v}_{t}+\boldsymbol{\vartheta}_{t}\right)
$$

where $\boldsymbol{\Psi}_{1}=\boldsymbol{\Phi}+\boldsymbol{\psi}_{1} \mathbf{W}$ and

$$
\mathbf{z}_{t}=\left(\mathbf{I}_{2 N}-\mathbf{\Psi}_{1} \mathbf{L}\right)^{-1} \boldsymbol{\beta} \boldsymbol{v}_{t}+\left(\mathbf{I}-\mathbf{\Psi}_{1} \mathbf{L}\right)^{-1} \boldsymbol{\vartheta}_{t}
$$

Note that by construction $\boldsymbol{v}_{t}$ and $\boldsymbol{\vartheta}_{t}$ are orthogonal, and for sufficiently large $p$, they are serially uncorrelated. Hence, the impulse response of shocks to elements of $\boldsymbol{v}_{t}$ and $\boldsymbol{\vartheta}_{t}$ can be computed using the following moving average representation:

$$
\mathbf{z}_{t}=\sum_{n=0}^{\infty} \mathbf{A}_{n} \boldsymbol{v}_{t-n}+\sum_{n=0}^{\infty} \mathbf{C}_{n} \boldsymbol{\vartheta}_{t-n}
$$

where $\mathbf{A}_{n}=\boldsymbol{\Psi}_{1}^{n} \boldsymbol{\beta}$, and $\mathbf{C}_{n}=\boldsymbol{\Psi}_{1}^{n}$, for $n=0,1,2, \ldots$

\section{S3.1 Responses to Common and Country-specific Shocks}

Let $\mathfrak{e}_{i}$ be a selection vector such that $\mathfrak{e}_{i}^{\prime} \mathbf{z}_{t}$ picks the $i^{\text {th }}$ element of $\mathbf{z}_{t}$. Also let $\mathbf{s}_{f}=(1,0)^{\prime}$ and $\mathbf{s}_{g}=(0,1)^{\prime}$, the vectors that select $\zeta_{t}$ and $\xi_{t}$ from $\boldsymbol{v}_{t}$, namely:

$$
\mathbf{s}_{f}^{\prime} \boldsymbol{v}_{t} \equiv \zeta_{t}, \quad \mathbf{s}_{g}^{\prime} \boldsymbol{v}_{t} \equiv \xi_{t}
$$

Recall now that $\zeta_{t}$ and $\xi_{t}$ have zero means, unit variances and are orthogonal to each other. Then the impulse responses to a positive unit shock to $\zeta_{t}$ or $\xi_{t}$ are given by:

$$
I R_{i, \zeta, n}=\mathfrak{e}_{i}^{\prime} \mathbf{A}_{n} \mathbf{s}_{f} \quad \text { and } I R_{i, \xi, n}=\mathfrak{e}_{i}^{\prime} \mathbf{A}_{n} \mathbf{s}_{g} \quad \text { for } n=0,1,2, \ldots
$$

where $\mathbf{A}_{n}$ is given by the moving average representation, (S3.4)

To derive impulse response functions for country-specific shocks, namely the $j^{\text {th }}$ element of $\boldsymbol{\vartheta}_{t}$, we need to make assumptions about the correlation between volatility and growth innovations within each country and across countries. Since the elements of $\boldsymbol{\vartheta}_{t}$ are weakly correlated across 
countries, they have some, but limited correlations across countries (see Figure 4). We also documented that, conditional on the common factors $\zeta_{t}$ and $\xi_{t}$, the country correlation of volatility and growth innovations are statistically insignificant for all except for four countries.

As a first order approximation, therefore, we will assume that the covariance matrix of $\boldsymbol{\vartheta}_{t}$ in (S3.3) is diagonal. Under this assumption, the impulse response function of a positive, unit shock to the $j^{t h}$ element of $\boldsymbol{\vartheta}_{t}$ on the the $i^{t h}$ element of $\mathbf{z}_{t}$ is given by:

$$
I R_{i, \vartheta_{j}, n}=\sqrt{\hat{\omega}_{j j}} \mathfrak{e}_{i}^{\prime} \mathbf{C}_{n} \mathfrak{e}_{j}
$$

where $\mathbf{C}_{n}$ is given by the moving average representation, (S3.4), $\hat{\omega}_{j j}$ is the (estimate) of the variance of the $j^{\text {th }}$ country-specific shock and $\mathfrak{e}_{j}$ is a selection vector such that $\mathfrak{e}_{j}^{\prime} \mathbf{z}_{t}$ picks the $j^{\text {th }}$ element of $\mathbf{z}_{t}$.

The above impulse responses can be compared to the generalized impulse responses of Pesaran and Shin (1998). The latter are given by:

$$
G I R_{i, \vartheta_{j}, n}=\frac{\mathfrak{e}_{i}^{\prime} \mathbf{C}_{n} \hat{\Omega} \mathfrak{e}_{j}}{\sqrt{\hat{\omega}_{j j}}},
$$

where $\hat{\Omega}=\left(\hat{\omega}_{i j}\right)$ is the estimate of the covariance of $\boldsymbol{\vartheta}_{t}$. The generalized impulse responses allow for non-zero correlations across the idiosyncratic errors. The two sets of impulse responses coincide if the covariance matrix of $\boldsymbol{\vartheta}_{t}$ is diagonal.

\section{S3.2 Forecast Error Variance Decompositions}

Traditionally, the forecast error variance decomposition of a VAR model is performed on a set of orthogonalized shocks, whereby the contribution of the $j^{\text {th }}$ orthogonalized innovation to the mean square error of the $n$-step ahead forecast of the model is calculated. In our empirical application this is not the case as - even if the country-specific volatility and growth innovations $\eta_{i t}$ and $\varepsilon_{i t}$ are weakly correlated across countries - some pairs of innovations can still display some non-zero correlation. An alternative approach is to compute Generalized Forecast Error Variance Decompositions (GVD) of Pesaran and Shin (1998). The Generalized Forecast Error Variance Decompositions consider the proportion of the variance of the $n$-step forecast errors of the endogenous variables that is explained by conditioning on the non-orthogonalized shocks, while explicitly allowing for the contemporaneous correlations between these shocks and the shocks to the other equations in the system.

Let $G V D_{i, \zeta, n}$ and $G V D_{i, \xi, n}$ be the share of the $n$-step ahead forecast error variance of the $i^{t h}$ variable in $\mathbf{z}_{t}$ that is accounted for by $\zeta_{t}$ and $\xi_{t}$, respectively, and $G V D_{i, j}$ the variance share 
of a generic country-specific shock, then:

$$
\begin{aligned}
G V D_{i, \zeta, n} & =\frac{\sum_{\ell=0}^{n}\left(\mathfrak{e}_{i}^{\prime} \mathbf{A}_{\ell} \mathbf{s}_{f}\right)^{2}}{\sum_{\ell=0}^{n} \mathfrak{e}_{i}^{\prime} \mathbf{A}_{\ell} \mathbf{A}_{\ell}^{\prime} \mathfrak{e}_{i}+\sum_{\ell=0}^{n} \mathfrak{e}_{i}^{\prime} \mathbf{C}_{\ell} \hat{\mathbf{\Omega}} \mathbf{C}_{\ell}^{\prime} \mathfrak{e}_{i}}, \quad n=1,2, \ldots, H, \\
G V D_{i, \xi, n} & =\frac{\sum_{\ell=0}^{n}\left(\mathfrak{e}_{i}^{\prime} \mathbf{A}_{\ell} \mathbf{s}_{g}\right)^{2}}{\sum_{\ell=0}^{n} \mathfrak{e}_{i}^{\prime} \mathbf{A}_{\ell} \mathbf{A}_{\ell}^{\prime} \mathfrak{e}_{i}+\sum_{\ell=0}^{n} \mathfrak{e}_{i}^{\prime} \mathbf{C}_{\ell} \hat{\mathbf{\Omega}} \mathbf{C}_{\ell}^{\prime} \mathfrak{e}_{i}}, \quad n=1,2, \ldots, H, \\
G V D_{i, j, n} & =\frac{\hat{\omega}_{j j}^{-1} \sum_{\ell=0}^{n}\left(\mathfrak{e}_{i}^{\prime} \mathbf{C}_{\ell} \hat{\mathbf{\Omega}}_{\mathfrak{e}_{j}}\right)^{2}}{\sum_{\ell=0}^{n} \mathfrak{e}_{i}^{\prime} \mathbf{A}_{\ell} \mathbf{A}_{\ell}^{\prime} \mathfrak{e}_{i}+\sum_{\ell=0}^{n} \mathfrak{e}_{i}^{\prime} \mathbf{C}_{\ell} \hat{\mathbf{\Omega}} \mathbf{C}_{\ell}^{\prime} \mathfrak{e}_{i}}, \quad j=1,2, \ldots, 2 N, \quad n=1,2, \ldots, H ;(\mathrm{S} 3.1,1
\end{aligned}
$$

Note that the different assumptions we make on the covariance matrix of all country-specific shocks, $\hat{\boldsymbol{\Omega}}$, have implications for the error variance decompositions. Specifically, when we assume that (i) $\hat{\boldsymbol{\Omega}}$ is diagonal or (ii) $\hat{\boldsymbol{\Omega}}$ is block-diagonal with Cholesky-orthogonalized blocks, the relative importance of shocks to country volatility and growth for all countries $\left(\eta_{i t}\right.$ and $\varepsilon_{i t}$, for $j=1,2, \ldots, 2 N)$ and shocks to the two common factors $\zeta_{t}$ and $\xi_{t}$, is easily characterized as

$V D_{i, \zeta, n}+V D_{i, \xi, n}+\sum_{j=1}^{2 N} V D_{i, j, n}=1$. That is the GVD formula coincides with the standard VD formula. In contrast, when we consider an unrestricted covariance matrix $\hat{\Omega}$, the sum of the variance shares does not necessarily add up to 1 .

\section{S3.3 Average Impulse Responses and Forecast Error Variance Decomposi- tions}

As a summary measure of the effects of shocks to the common factors we report the following average measures. Denote the impulse response (or forecast error variance decomposition) of a particular shock on the $j^{t h}$ variable in country $i$ at horizon $n$ by $X_{i, j, n}$. Let $w=\left(w_{1}, w_{2}, \ldots, w_{N}\right)^{\prime}$ be a vector of fixed weights such that $\Sigma_{i=1}^{N} w_{i}=1$. Then the average impulse response (or forecast error variance decomposition) of the shock to variable $j$, at horizon $n$, is computed as:

$$
X_{\omega, j, n}=\sum_{i=1}^{N} w_{i} X_{i, j, n} .
$$

and its dispersion is computed by:

$$
\sigma_{X_{\omega, j, n}}=\left[\sum_{i=1}^{N} w_{i}^{2}\left(X_{i, j, n}-X_{\omega, j, n}\right)^{2}\right]^{1 / 2}
$$

assuming country-specific impulse responses or forecast error variance decompositions are approximately uncorrelated. 


\section{S4 Country-specific Results}

In this section we report selected country-specific results, including summary statistics, and the individual forecast error variance decompositions.

Figure S.5 plots the US realized volatility measure we constructed with the VIX index (during the period over which they overlap). The chart shows that the two measures co-move very closely with a correlation coefficient of around 0.9 .

Figure S.5 Estimated Quarterly US Equity Realized Volatility AND THE VIX INDEX

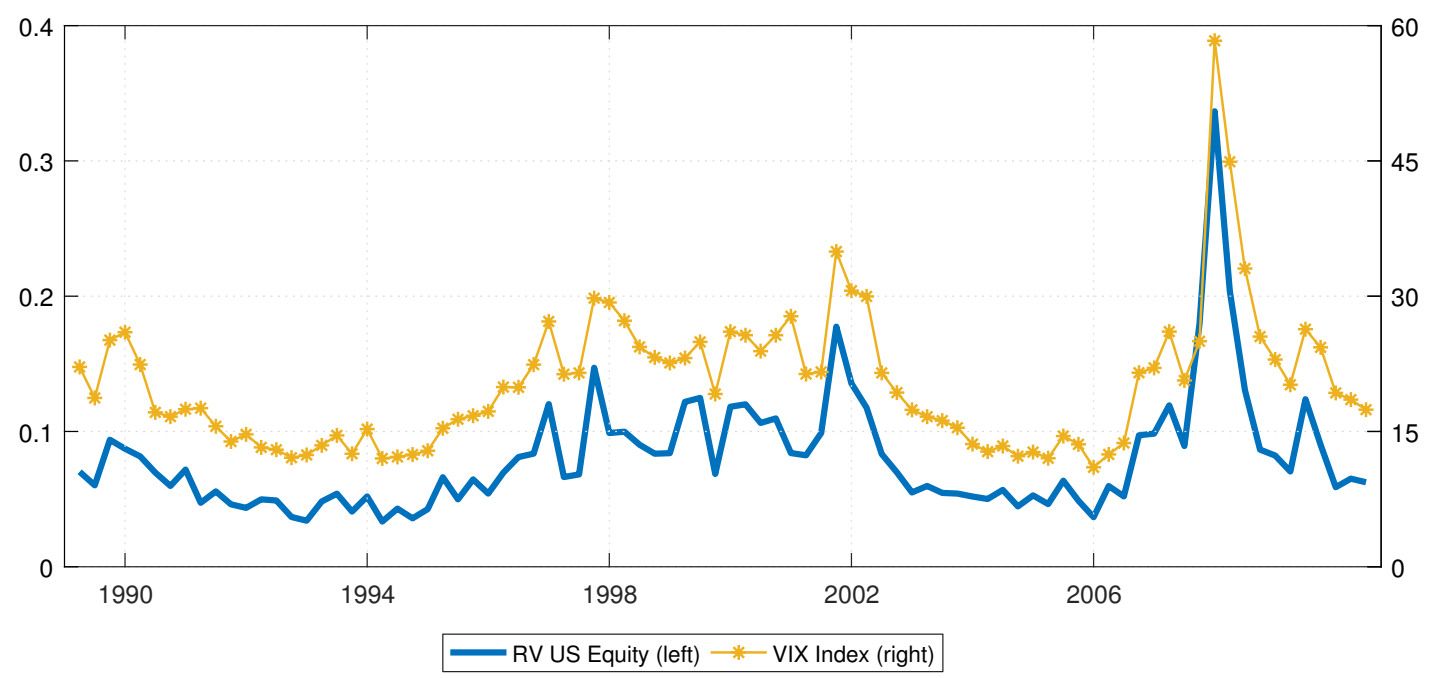

Note. 'RV US Equity' is the US realized volatility measure defined by (64). The VIX Index is the quarterly average of the daily Chicago Board Options Exchange Market Volatility Index from Bloomberg. Sample period: 1990:Q1-2011:Q2.

Figures S.6 to S.11 report forecast error variance decompositions for each country, for both volatility and growth, computed with different assumptions on the covariance matrix of the volatility and growth innovations. As can be seen the estimates are very similar across countries and for all the three schemes assumed for the error covariances.

Figure S.12 plots the country-specific impulse response of volatility and growth to a positive, one-standard-deviation shock to the common shocks $\hat{\zeta}_{t}$ and $\hat{\xi}_{t}$. We can see from Figure S.12 that for most countries the impulse responses have a very similar profile. 


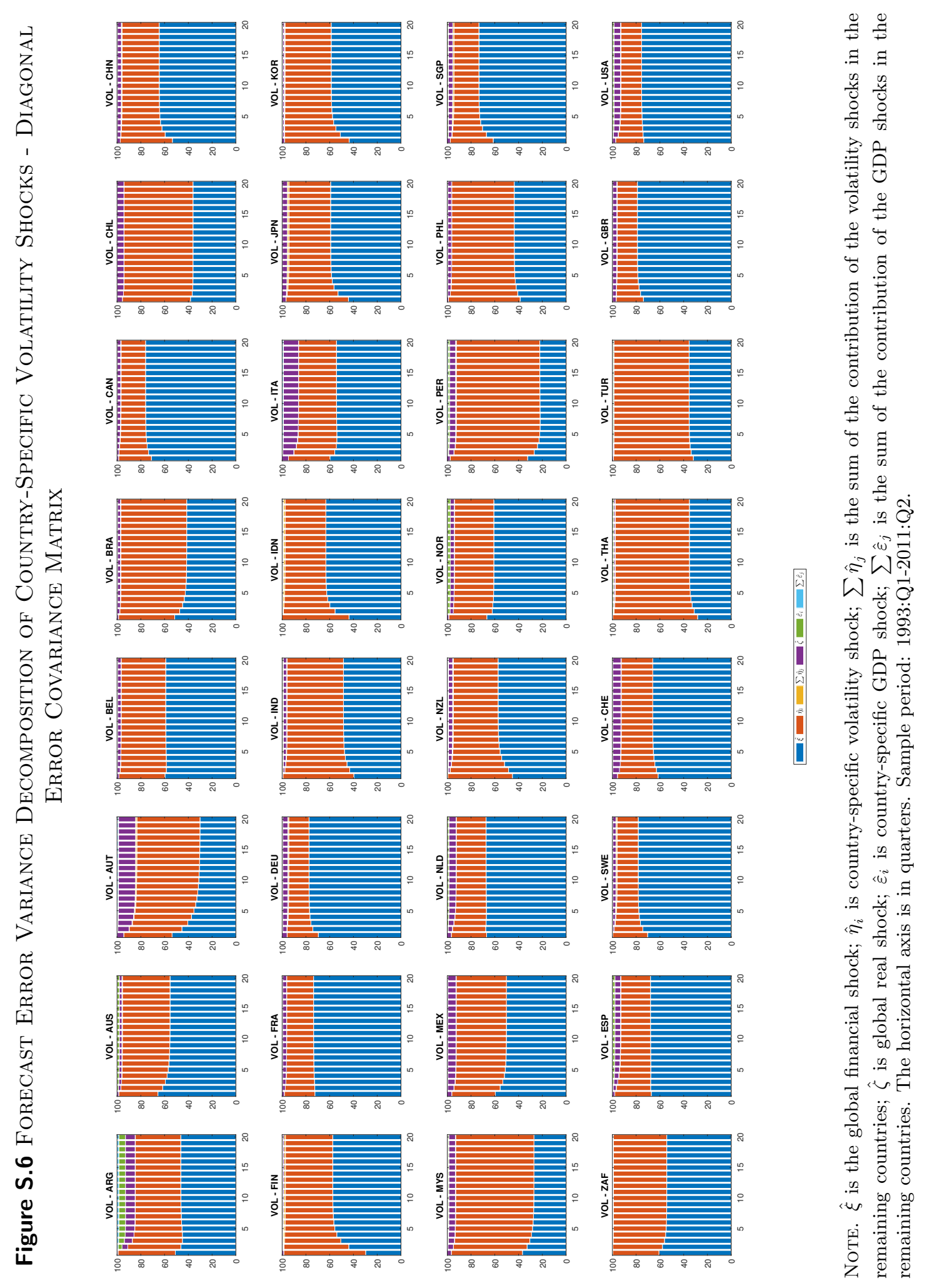




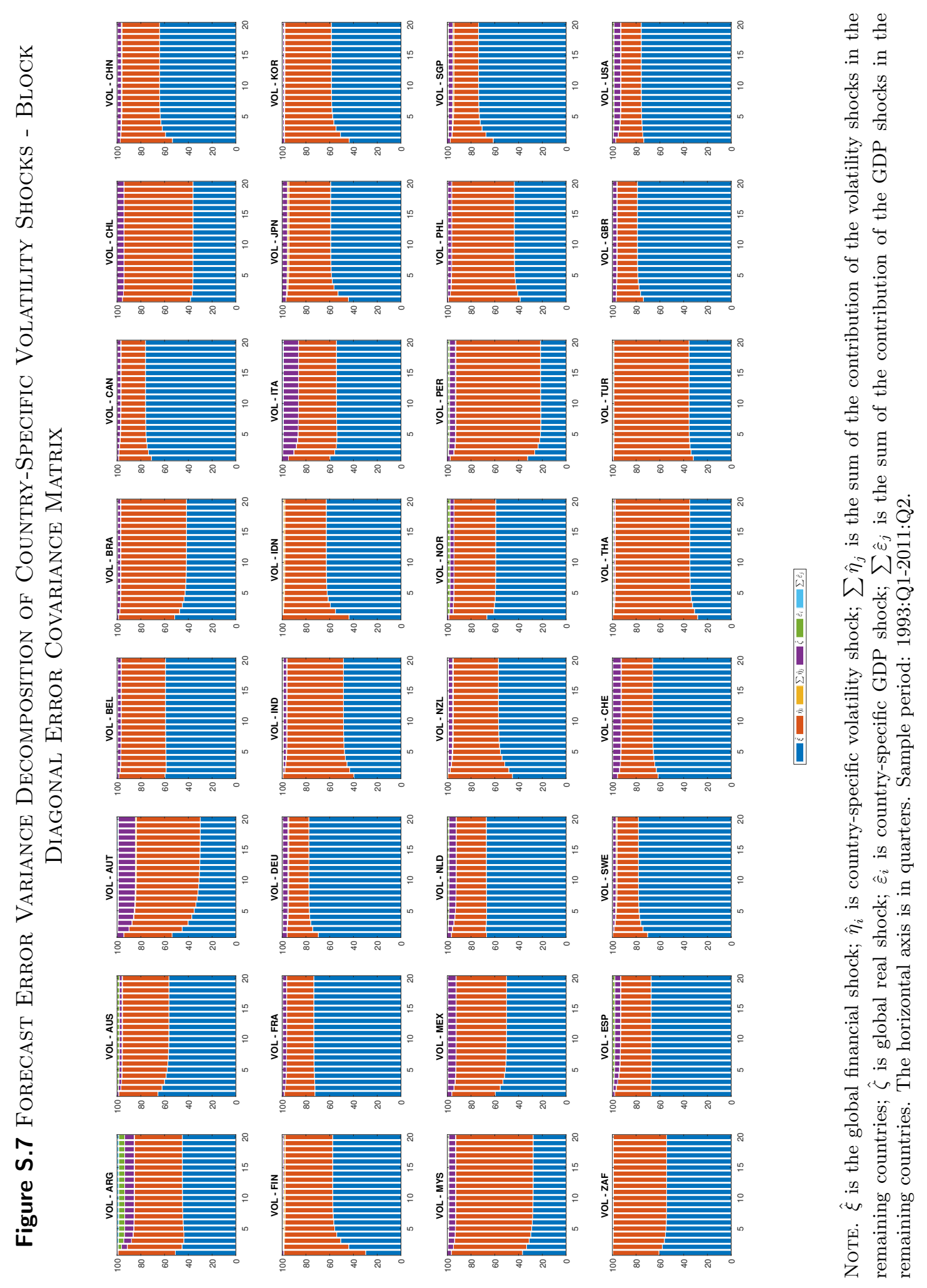




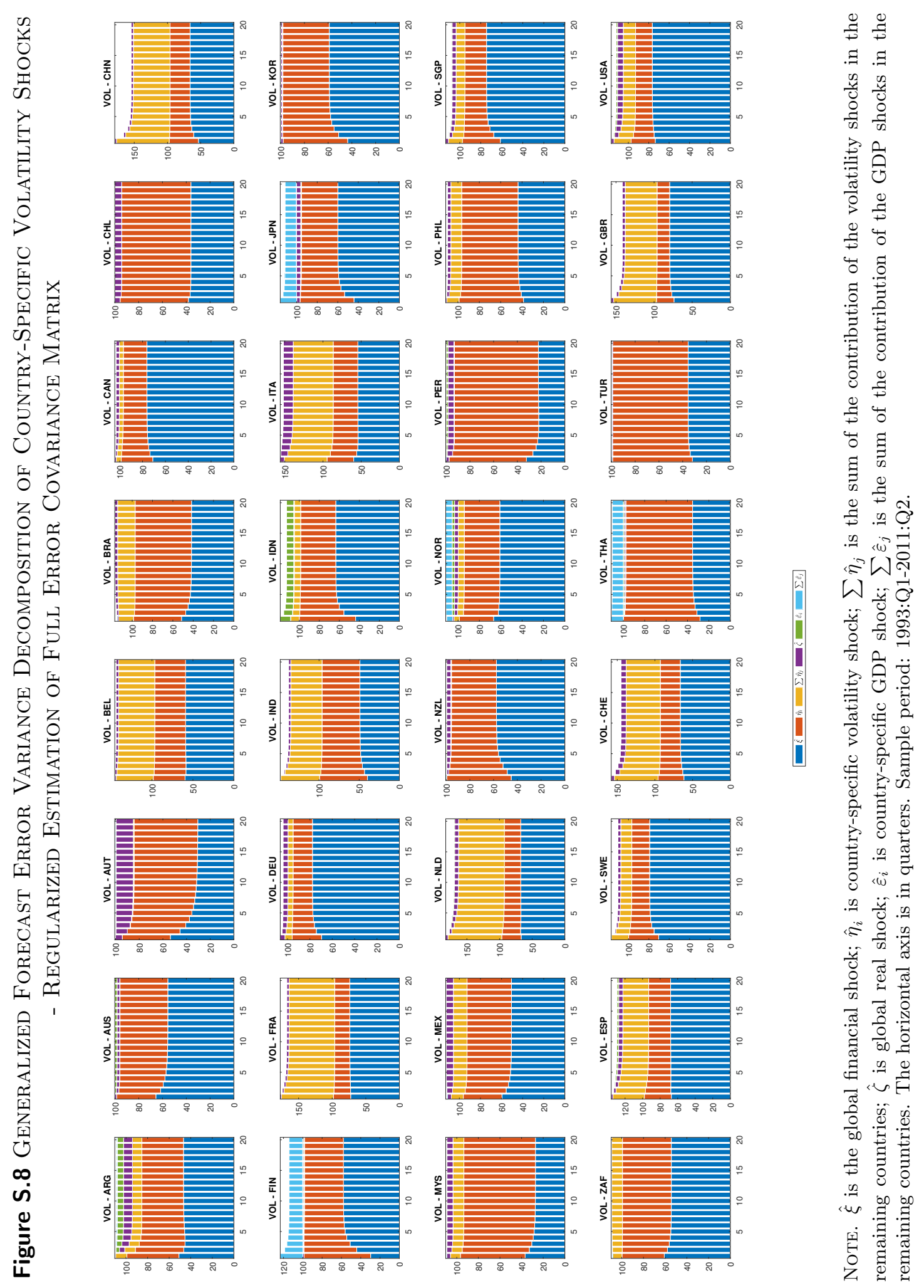




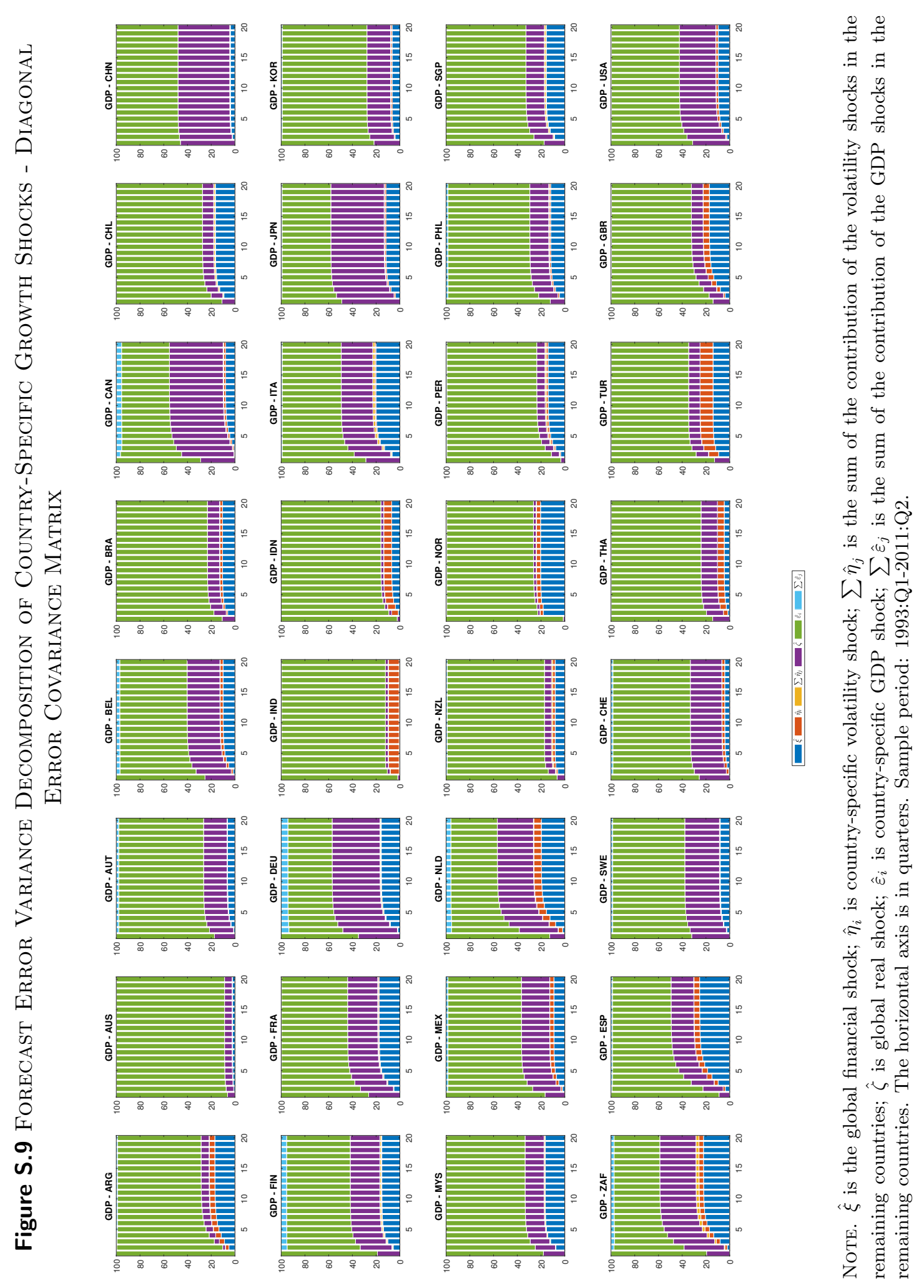




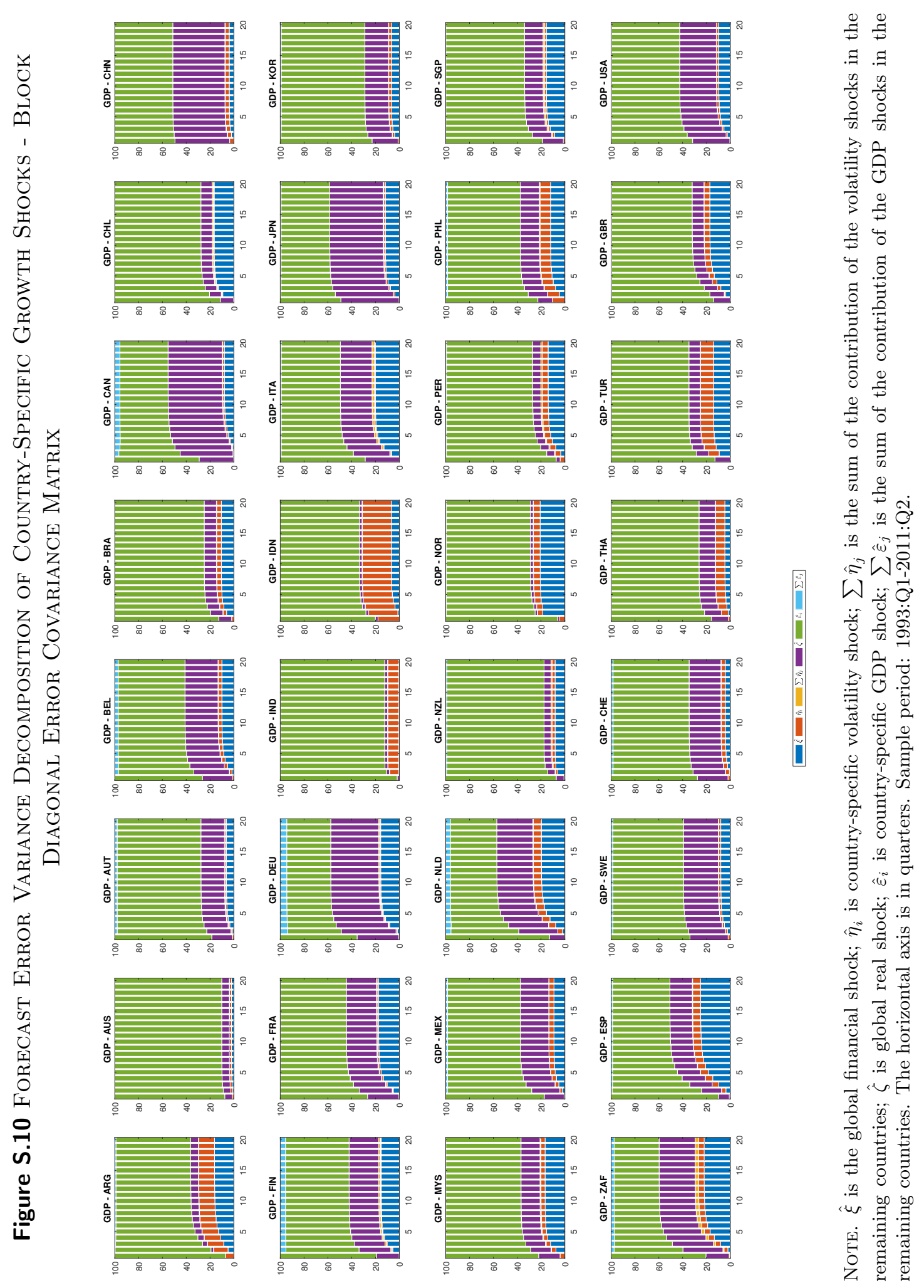




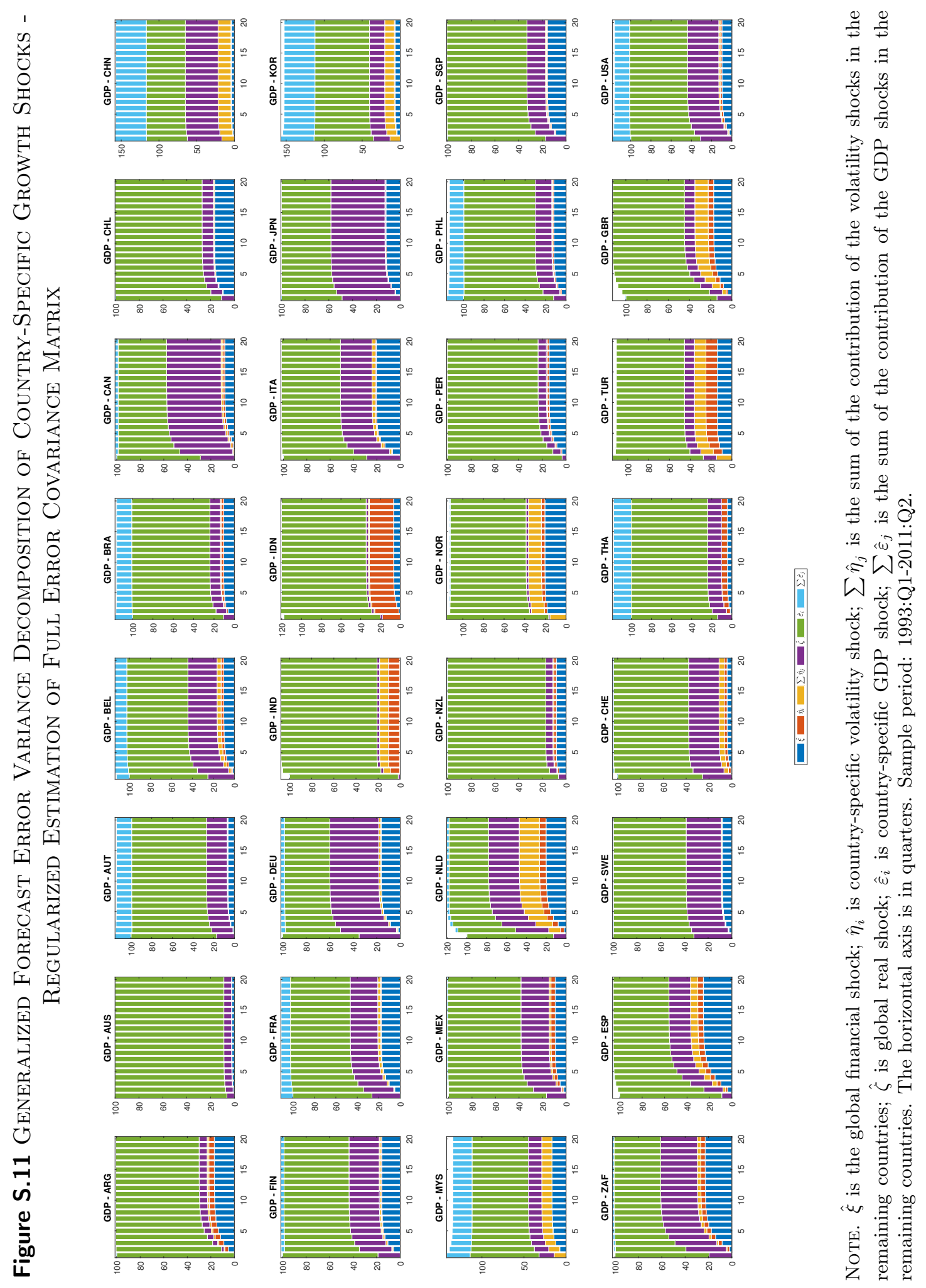


Figure S.12 Country-specific Volatility and Growth Impulse Responses to Common Real and Financial Shocks

(A) $v_{i t}$ to a $\zeta_{t}$ shock

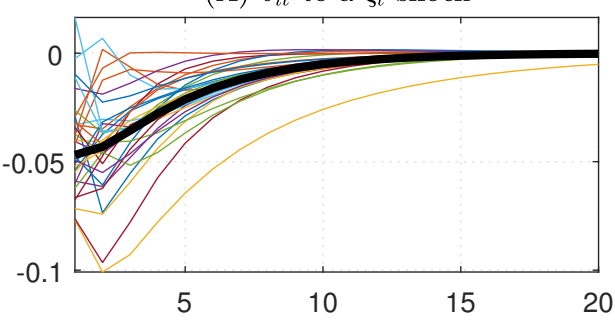

(C) $v_{i t}$ to a $\xi_{t}$ shock

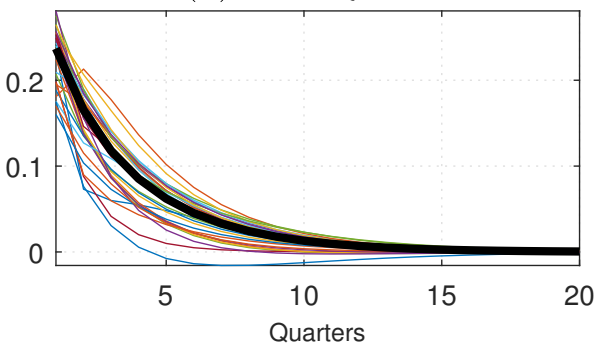

(B) $\Delta y_{i t}$ to a $\zeta_{t}$ shock

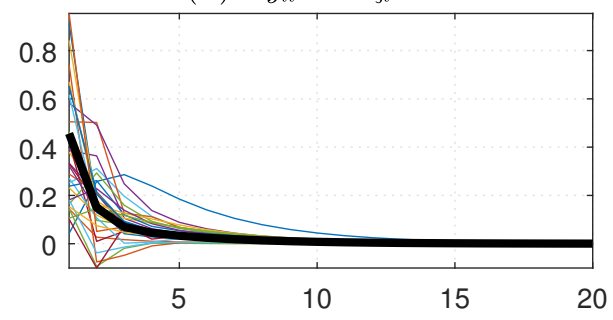

(D) $\Delta y_{i t}$ to a $\xi_{t}$ shock

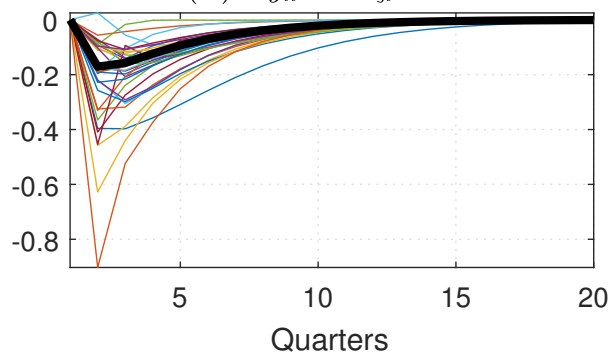

Note. One standard deviation shocks to $\hat{\zeta}_{t}$ and $\hat{\xi}_{t}$. Thin lines are individual country responses. The solid lines are the PPP-GDP weighted averages, as the ones reported in the main text. Impulse responses are computed as in Appendix S3. Sample period: 1993:Q1-2011:Q2. 


\section{S5 Other results}

Tables S.1, S.2, and S.3 report the summary statistics for the realized volatility series, the loglevel of real GDP, and the log-difference of real GDP for each country in our sample. These

results support the use of GDP growth and log-level of realized volatilities as stationary series in our empirical analysis.

Table S.1 Summary Statistics for Country-specific Realized Volatility (LOG-LEVEL)

\begin{tabular}{|c|c|c|c|c|c|c|c|c|c|c|c|}
\hline & $\mathrm{ARG}$ & AUS & AUT & BEL & BRA & CAN & CHL & $\mathrm{CHN}$ & FIN & FRA & DEU \\
\hline Obs & 94 & 129 & 129 & 129 & 86 & 129 & 94 & 74 & 94 & 129 & 129 \\
\hline Mean & -1.72 & -2.63 & -2.88 & -2.79 & -1.67 & -2.73 & -2.54 & -2.01 & -2.07 & -2.52 & -2.46 \\
\hline Max & 0.00 & -1.07 & -1.17 & -1.41 & 0.61 & -1.08 & -1.44 & -0.88 & -0.26 & -1.27 & -1.09 \\
\hline Min & -2.58 & -3.40 & -4.22 & -3.59 & -2.72 & -3.44 & -3.17 & -2.71 & -3.19 & -3.23 & -3.29 \\
\hline St. Dev. & 0.51 & 0.35 & 0.63 & 0.45 & 0.65 & 0.44 & 0.36 & 0.42 & 0.54 & 0.38 & 0.34 \\
\hline Auto Corr. & 0.63 & 0.51 & 0.78 & 0.61 & 0.84 & 0.70 & 0.47 & 0.64 & 0.78 & 0.53 & 0.54 \\
\hline \multirow[t]{2}{*}{$\mathrm{ADF}$} & $-2.95^{b}$ & $-2.97^{b}$ & $-2.73^{c}$ & $-3.35^{b}$ & -2.45 & $-2.78^{c}$ & $-3.63^{a}$ & -2.57 & $-2.72^{c}$ & $-3.25^{b}$ & $-3.71^{a}$ \\
\hline & IND & IDN & ITA & JPN & KOR & MYS & MEX & NLD & NZL & NOR & PER \\
\hline Obs & 97 & 94 & 129 & 129 & 129 & 125 & 94 & 129 & 94 & 125 & 74 \\
\hline Mean & -2.19 & -2.12 & -2.39 & -2.50 & -2.24 & -2.51 & -2.22 & -2.64 & -2.52 & -2.31 & -2.09 \\
\hline Max & -1.23 & -0.56 & -1.27 & -1.07 & -1.08 & -0.75 & -1.21 & -1.21 & -1.44 & -0.93 & -0.80 \\
\hline Min & -2.92 & -3.19 & -3.32 & -3.67 & -3.52 & -3.64 & -2.97 & -3.36 & -3.28 & -3.05 & -2.79 \\
\hline St. Dev. & 0.39 & 0.50 & 0.41 & 0.44 & 0.44 & 0.50 & 0.35 & 0.43 & 0.37 & 0.36 & 0.40 \\
\hline Auto Corr. & 0.52 & 0.55 & 0.60 & 0.64 & 0.72 & 0.61 & 0.55 & 0.67 & 0.65 & 0.54 & 0.62 \\
\hline \multirow[t]{2}{*}{$\mathrm{ADF}$} & $-3.19^{b}$ & -2.09 & $-3.49^{a}$ & $-3.85^{a}$ & -2.48 & $-3.18^{b}$ & $-2.74^{c}$ & $-2.87^{c}$ & $-2.69^{c}$ & $-3.2^{b}$ & -2.06 \\
\hline & PHL & SGP & $\mathrm{ZAF}$ & ESP & SWE & $\mathrm{CHE}$ & THA & TUR & GBR & USA & \\
\hline Obs & 101 & 129 & 129 & 129 & 117 & 129 & 97 & 94 & 129 & 129 & \\
\hline Mean & -2.19 & -2.51 & -2.21 & -2.58 & -2.37 & -2.85 & -2.11 & -1.65 & -2.61 & -2.63 & \\
\hline Max & -0.82 & -0.95 & -0.89 & -1.22 & -1.21 & -1.41 & -1.20 & -0.87 & -1.23 & -1.09 & \\
\hline Min & -3.13 & -3.29 & -3.27 & -3.49 & -3.13 & -3.89 & -2.85 & -2.60 & -3.43 & -3.40 & \\
\hline St. Dev. & 0.39 & 0.44 & 0.40 & 0.46 & 0.39 & 0.50 & 0.40 & 0.36 & 0.39 & 0.40 & \\
\hline Auto Corr. & 0.50 & 0.55 & 0.45 & 0.67 & 0.59 & 0.64 & 0.53 & 0.61 & 0.55 & 0.68 & \\
\hline $\mathrm{ADF}$ & $-4.1^{a}$ & $-3.27^{b}$ & $-3.72^{a}$ & $-3.01^{b}$ & $-3.13^{b}$ & $-3.02^{b}$ & $-3.31^{b}$ & -1.76 & $-3.24^{b}$ & $-2.83^{c}$ & \\
\hline
\end{tabular}

Note. Summary statistics of the log-level of volatility $\left(v_{i t}\right)$. ADF is the Augmented Dickey-Fuller t-statistic computed with 4 lags and a constant, where $a, b$, and $c$ denote associated p-values at 1\%, $5 \%$, and $10 \%$. 
Table S.2 Summary Statistics for Country-specific Real GDP (Log-Level)

\begin{tabular}{|c|c|c|c|c|c|c|c|c|c|c|c|}
\hline & $\mathrm{ARG}$ & AUS & AUT & BEL & BRA & $\mathrm{CAN}$ & CHL & $\mathrm{CHN}$ & FIN & FRA & DEU \\
\hline Obs & 128 & 128 & 128 & 128 & 128 & 128 & 128 & 128 & 128 & 128 & 128 \\
\hline Mean & 0.65 & 0.80 & 0.54 & 0.48 & 0.70 & 0.62 & 1.15 & 2.49 & 0.57 & 0.44 & 0.42 \\
\hline Max & 5.18 & 2.85 & 3.48 & 2.23 & 5.16 & 2.47 & 8.39 & 5.91 & 4.41 & 1.56 & 2.63 \\
\hline Min & -6.35 & -1.65 & -2.61 & -2.12 & -7.25 & -2.27 & -6.72 & -1.33 & -6.01 & -1.70 & -4.16 \\
\hline St. Dev. & 2.20 & 0.78 & 0.97 & 0.78 & 1.88 & 0.79 & 2.07 & 1.21 & 1.45 & 0.52 & 0.94 \\
\hline Auto Corr. & 0.59 & 0.29 & -0.03 & 0.26 & 0.21 & 0.55 & 0.27 & 0.27 & 0.07 & 0.40 & 0.13 \\
\hline \multirow[t]{2}{*}{$\mathrm{ADF}$} & -4.17 & -4.59 & -4.41 & -5.00 & -5.42 & -4.22 & -5.05 & -3.63 & -3.82 & -4.02 & -4.52 \\
\hline & IND & IDN & ITA & JPN & KOR & MYS & MEX & NLD & NZL & NOR & PER \\
\hline Obs & 128 & 128 & 128 & 128 & 128 & 128 & 128 & 128 & 128 & 128 & 128 \\
\hline Mean & 1.53 & 1.24 & 0.36 & 0.45 & 1.45 & 1.42 & 0.65 & 0.50 & 0.52 & 0.67 & 0.77 \\
\hline Max & 3.59 & 12.08 & 2.84 & 2.68 & 6.67 & 5.22 & 3.79 & 2.98 & 3.47 & 4.68 & 7.04 \\
\hline Min & -2.84 & -8.17 & -3.70 & -4.09 & -8.94 & -7.10 & -6.07 & -2.38 & -2.72 & -3.48 & -14.00 \\
\hline St. Dev. & 1.06 & 2.19 & 0.73 & 1.02 & 1.82 & 1.61 & 1.58 & 0.78 & 0.96 & 1.27 & 3.11 \\
\hline Auto Corr. & 0.27 & 0.02 & 0.36 & 0.29 & -0.01 & 0.35 & 0.20 & 0.23 & 0.20 & -0.24 & 0.38 \\
\hline \multirow[t]{2}{*}{$\mathrm{ADF}$} & -5.27 & -4.80 & -4.11 & $-4.14^{c}$ & $-4.87^{c}$ & -5.26 & -4.84 & -3.54 & -4.48 & -3.56 & -4.34 \\
\hline & PHL & SGP & ZAF & ESP & SWE & $\mathrm{CHE}$ & THA & TUR & GBR & USA & \\
\hline Obs & 128 & 128 & 128 & 128 & 128 & 128 & 128 & 128 & 128 & 128 & \\
\hline Mean & 0.79 & 1.65 & 0.61 & 0.58 & 0.53 & 0.44 & 1.31 & 1.00 & 0.48 & 0.64 & \\
\hline Max & 4.63 & 6.77 & 2.50 & 2.49 & 4.41 & 2.50 & 6.06 & 7.03 & 2.18 & 2.23 & \\
\hline Min & -6.88 & -3.77 & -2.14 & -1.57 & -5.12 & -3.50 & -5.11 & -11.93 & -2.40 & -2.18 & \\
\hline St. Dev. & 1.55 & 1.91 & 0.86 & 0.56 & 1.32 & 0.83 & 1.65 & 2.68 & 0.76 & 0.76 & \\
\hline Auto Corr. & 0.12 & 0.23 & 0.58 & 0.80 & -0.23 & 0.24 & 0.49 & 0.04 & 0.52 & 0.41 & \\
\hline $\mathrm{ADF}$ & -3.17 & -5.75 & -4.33 & -3.09 & -4.50 & -4.31 & -3.11 & -6.31 & -4.60 & -4.43 & \\
\hline
\end{tabular}

Note. Summary statistics for the log-level of real GDP $\left(y_{i t}\right)$. ADF is the Augmented Dickey-Fuller t-statistic computed with 4 lags and a constant, where $a, b$, and $c$ denote associated p-values at 1\%, $5 \%$, and $10 \%$. 
Table S.3 Summary Statistics for Country-Specific Real GDP (Log-Difference)

\begin{tabular}{|c|c|c|c|c|c|c|c|c|c|c|c|}
\hline & ARG & AUS & AUT & BEL & BRA & $\mathrm{CAN}$ & $\mathrm{CHL}$ & $\mathrm{CHN}$ & FIN & FRA & DEU \\
\hline Obs & 128 & 128 & 128 & 128 & 128 & 128 & 128 & 128 & 128 & 128 & 128 \\
\hline Mean & 0.65 & 0.80 & 0.54 & 0.48 & 0.70 & 0.62 & 1.15 & 2.49 & 0.57 & 0.44 & 0.42 \\
\hline Max & 5.18 & 2.85 & 3.48 & 2.23 & 5.16 & 2.47 & 8.39 & 5.91 & 4.41 & 1.56 & 2.63 \\
\hline Min & -6.35 & -1.65 & -2.61 & -2.12 & -7.25 & -2.27 & -6.72 & -1.33 & -6.01 & -1.70 & -4.16 \\
\hline St. Dev. & 2.20 & 0.78 & 0.97 & 0.78 & 1.88 & 0.79 & 2.07 & 1.21 & 1.45 & 0.52 & 0.94 \\
\hline Auto Corr. & 0.59 & 0.29 & -0.03 & 0.26 & 0.21 & 0.55 & 0.27 & 0.27 & 0.07 & 0.40 & 0.13 \\
\hline \multirow[t]{2}{*}{$\mathrm{ADF}$} & $-4.17^{a}$ & $-4.59^{a}$ & $-4.41^{a}$ & $-5^{a}$ & $-5.42^{a}$ & $-4.22^{a}$ & $-5.05^{a}$ & $-3.63^{a}$ & $-3.82^{a}$ & $-4.02^{a}$ & $-4.52^{a}$ \\
\hline & IND & IDN & ITA & JPN & KOR & MYS & MEX & NLD & NZL & NOR & PER \\
\hline Obs & 128 & 128 & 128 & 128 & 128 & 128 & 128 & 128 & 128 & 128 & 128 \\
\hline Mean & 1.53 & 1.24 & 0.36 & 0.45 & 1.45 & 1.42 & 0.65 & 0.50 & 0.52 & 0.67 & 0.77 \\
\hline Max & 3.59 & 12.08 & 2.84 & 2.68 & 6.67 & 5.22 & 3.79 & 2.98 & 3.47 & 4.68 & 7.04 \\
\hline Min & -2.84 & -8.17 & -3.70 & -4.09 & -8.94 & -7.10 & -6.07 & -2.38 & -2.72 & -3.48 & -14.00 \\
\hline St. Dev. & 1.06 & 2.19 & 0.73 & 1.02 & 1.82 & 1.61 & 1.58 & 0.78 & 0.96 & 1.27 & 3.11 \\
\hline Auto Corr. & 0.27 & 0.02 & 0.36 & 0.29 & -0.01 & 0.35 & 0.20 & 0.23 & 0.20 & -0.24 & 0.38 \\
\hline \multirow[t]{2}{*}{$\mathrm{ADF}$} & $-5.27^{a}$ & $-4.8^{a}$ & $-4.11^{a}$ & $-4.14^{a}$ & $-4.87^{a}$ & $-5.26^{a}$ & $-4.84^{a}$ & $-3.54^{a}$ & $-4.48^{a}$ & $-3.56^{a}$ & $-4.34^{a}$ \\
\hline & PHL & SGP & $\mathrm{ZAF}$ & ESP & SWE & $\mathrm{CHE}$ & THA & TUR & GBR & USA & \\
\hline Obs & 128 & 128 & 128 & 128 & 128 & 128 & 128 & 128 & 128 & 128 & \\
\hline Mean & 0.79 & 1.65 & 0.61 & 0.58 & 0.53 & 0.44 & 1.31 & 1.00 & 0.48 & 0.64 & \\
\hline Max & 4.63 & 6.77 & 2.50 & 2.49 & 4.41 & 2.50 & 6.06 & 7.03 & 2.18 & 2.23 & \\
\hline Min & -6.88 & -3.77 & -2.14 & -1.57 & -5.12 & -3.50 & -5.11 & -11.93 & -2.40 & -2.18 & \\
\hline St. Dev. & 1.55 & 1.91 & 0.86 & 0.56 & 1.32 & 0.83 & 1.65 & 2.68 & 0.76 & 0.76 & \\
\hline Auto Corr. & 0.12 & 0.23 & 0.58 & 0.80 & -0.23 & 0.24 & 0.49 & 0.04 & 0.52 & 0.41 & \\
\hline $\mathrm{ADF}$ & $-3.17^{b}$ & $-5.75^{a}$ & $-4.33^{a}$ & $-3.09^{b}$ & $-4.5^{a}$ & $-4.31^{a}$ & $-3.11^{b}$ & $-6.31^{a}$ & $-4.6^{a}$ & $-4.43^{a}$ & \\
\hline
\end{tabular}

Note. Summary statistics for the log-difference of real GDP $\left(\Delta y_{i t}\right) . A D F$ is the Augmented Dickey-Fuller t-statistic computed with 4 lags and a constant, where $a, b$, and $c$ denote associated p-values at $1 \%, 5 \%$, and $10 \%$. 